\title{
Calibration and in-orbit performance of the reflection grating spectrometer onboard XMM-Newton
}

\author{
C. P. de Vries ${ }^{1}$, J. W. den Herder ${ }^{1}$, C. Gabriel ${ }^{2}$, R. Gonzalez-Riestra ${ }^{2}$, A. Ibarra ${ }^{2}$, J. S. Kaastra ${ }^{1}$, A. M. T. Pollock ${ }^{2}$, \\ A. J. J. Raassen ${ }^{1,4}$, and F. B. S. Paerels ${ }^{3}$ \\ ${ }^{1}$ SRON Netherlands Institute for Space Research, Sorbonnelaan 2, 3584 CA Utrecht, The Netherlands \\ e-mail: C.P.de.Vries@sron.nl \\ 2 European Space Agency, ESAC, Apartado 50727, 28080 Madrid, Spain \\ 3 Columbia Astrophysics Laboratory, 550 West 120th Street, New York, NY 10027, USA \\ 4 Astronomical Institute “Anton Pannekoek”, Science Park 904, University of Amsterdam, 1098 XH Amsterdam, The Netherlands
}

Received 24 February 2014 / Accepted 16 October 2014

\begin{abstract}
Context. XMM-Newton was launched on 10 December 1999 and has been operational since early 2000. One of the instruments onboard XMM-Newton is the reflection grating spectrometer (RGS). Two identical RGS instruments are available, with each RGS combining a reflection grating assembly and a camera with charge-coupled devices to record the spectra.

Aims. We describe the calibration and in-orbit performance of the RGS instrument. By combining the preflight calibration with appropriate inflight calibration data including the changes in detector performance over time, we aim at profound knowledge about the accuracy in the calibration. This will be crucial for any correct scientific interpretation of spectral features for a wide variety of objects.

Methods. Ground calibrations alone are not able to fully characterize the instrument. Dedicated inflight measurements and constant monitoring are essential for a full understanding of the instrument and the variations of the instrument response over time. Physical models of the instrument are tuned to agree with calibration measurements and are the basis from which the actual instrument response can be interpolated over the full parameter space.

Results. Uncertainties in the instrument response have been reduced to $<10 \%$ for the effective area and $<6 \mathrm{~m} \AA$ for the wavelength scale (in the range from $8 \AA$ to $34 \AA$ ). The remaining systematic uncertainty in the detection of weak absorption features has been estimated to be $1.5 \%$.

Conclusions. Based on a large set of inflight calibration data and comparison with other instruments onboard XMM-Newton, the calibration accuracy of the RGS instrument has been improved considerably over the preflight calibrations.
\end{abstract}

Key words. instrumentation: spectrographs - instrumentation: detectors - techniques: spectroscopic

\section{Introduction}

Accurate understanding of the instrument response is a prerequisite for correctly interpreting the observational data from $X M M-N e w t o n$. This understanding requires a profound knowledge of the limitations of the preflight calibrations and the models applied to calculate the response in orbit. In addition, changes in the calibration due to the operational conditions in space (radiation damage, contamination, etc.) should be understood well and be taken into account. Apart from statistical fluctuations in the data, important scientific results also depend on understanding the systematic errors of the instrument. With the simultaneous operations of two reflection grating spectrometer (RGS) instruments, we have an excellent tool for studying systematic errors. If the instrument model is correct, the two RGS instruments should produce consistent results within the statistical uncertainty. By using high signal-to-noise observations we can test this in detail.

The prime goal of the RGS instrument is to measure the emission lines and absorption features of specific transitions in highly ionized plasmas. In addition to a reasonable effective area, this requires especially good spectral resolving power $(R>250)$. Clearly, identifying and quantifying weak absorption or emission features is very challenging. For example, weak absorption features due to the warm hot intergalactic medium (WHIM) in the direction of the bright blazar Mrk 421 have been reported and disputed (Nicastro et al. 2005; Kaastra et al. 2006; Rasmussen et al. 2007). Because these detections have a typical significance between 3 , and $5 \sigma$, an accurate calibration of the instrument response is essential. Also studying the velocity broadening of lines critically depends on understanding the response (see Sect. 7). Knowing the time evolution of the response is important as well. The time-dependent change in the spectrum of the X-ray emission from the isolated neutron star RXJ 0720.4-3125 (de Vries et al. 2004; Haberl et al. 2006) could only be detected using detailed knowledge about the stability of the RGS response.

In this paper we give a quantitative assessment of the calibration of the RGS instrument onboard XMM-Newton. In Sect. 2 we present the main characteristics of the instrument. In Sect. 3 we describe the calibration method and data processing steps applied in the current data processing system. This is followed by the main part of this paper where we describe the preflight and inflight calibration for the various key instrumental characteristics (effective area, line spread function, wavelength calibration, and background). In the last Sects. 7-9 we summarize the current status of the calibrations and give an overview (Sect. 11) of relevant operational information. 


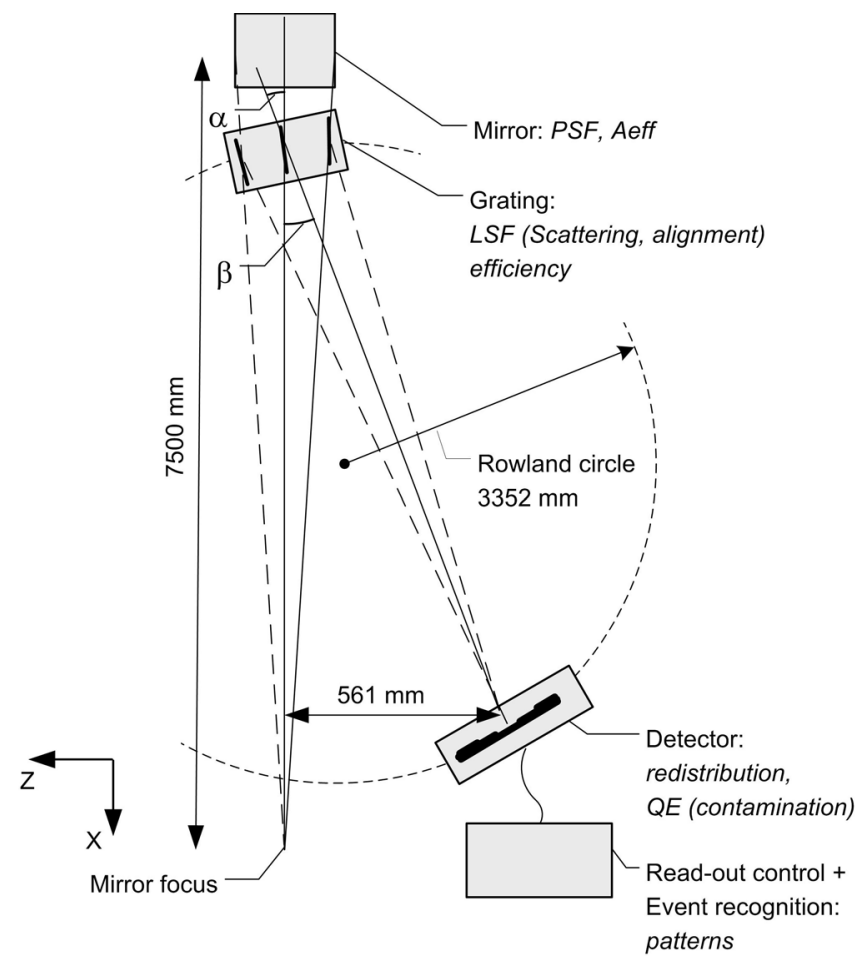

Fig. 1. Schematics of the RGS configuration (not to scale).

Since this paper aims to give a total overview of RGS performance and calibration, it is in part also a review of papers and calibration and operational documents published at various places elsewhere, in particular at the XMM-Newton science center at ESAC. Besides new information and data also previously published data are shown, when appropriate, to present a complete picture.

The latest calibration data are used by the standard data processing package for XMM-Newton, the science analysis system (SAS), which is maintained and can be downloaded from the $X M M-N e w t o n$ Science Operations Center at ESAC. In particular the "Calibration access and data handbook" offers many details on the calibrations and algorithms used.

\section{Instrument}

Both RGS instruments (RGS1 and RGS2) consist of a set of reflection gratings placed in the converging beam of the telescope combined with a camera in the spectroscopic focus. Detailed information about the instrument is given in den Herder et al. (2001) but some key features of the instrument, that are important to understand the accuracy of the calibrations are repeated here. The instrument is shown in Fig. 1 where the main components are given including the related calibration dependencies. The mirror response is characterized by its effective area $\left(A_{\mathrm{eff}}\right)$ and its point spread function (PSF). In the converging beam the reflection gratings are positioned on the Rowland circle. The response of the gratings can be described by their reflection efficiency, the scattering and the grating-to-grating alignment. The reflected X-rays are recorded on a photon-by-photon basis in the CCD detector where the position corresponds to the wavelength (energy) and the energy resolution of the CCDs is used to separate orders. The performance of these detectors will vary over the mission lifetime due to radiation damage (e.g. change in the CCD redistribution function) and due to contamination on the detectors (change in quantum efficiency). Finally X-ray event
Table 1. Key design parameters.

\begin{tabular}{lrl}
\hline \hline Parameter & Value & Comment \\
\hline$\lambda_{\text {blaze }}$ & $15 \AA$ & first order blaze wavelength \\
$\delta$ & $0.6989^{\circ}$ & blaze angle of facets \\
$\gamma$ & $2.2751^{\circ}$ & graze angle on facets \\
$\alpha$ & $1.5762^{\circ}$ & angle of incidence \\
$\beta_{\text {blaze }}$ & $2.9739^{\circ}$ & diffraction angle for $\lambda_{\text {blaze }}$ \\
$1 / d$ & 645.6 lines $/ \mathrm{mm}$ & central groove density \\
CCDs & 9 & back illuminated, $\sim 30 \mu \mathrm{m}$ thick \\
Pixels & $27 \times 27 \mu \mathrm{m}^{2}$ & pixel size; standard $3 \times 3$ on \\
& & chip binning \\
CCD size & $1024 \times 384$ & image area, identical storage \\
& & area \\
$T$ & -80 to $-110{ }^{\circ} \mathrm{C}$ & \\
\hline$\lambda$-range & $6.0-38 \AA$ & \\
$A_{\text {eff }}$ & $140 \mathrm{~cm}^{2}$ & for two units at $15 \AA$ \\
Resolving power & $100-500$ & $\approx 250$ at $15 \AA$ \\
$\Delta \lambda$ & $8 \mathrm{m \AA}$ & wavelength accuracy \\
\hline
\end{tabular}

patterns on the CCDs are recognized by the read-out unit. The european photon imaging camera (EPIC-MOS) is located in the focus of the mirrors.

The central position of the X-rays on the detector is given by the dispersion equation through:

$m \lambda=d(\cos \beta-\cos \alpha)$

where $m$ is the spectral order $(-1,-2), d$ is the groove spacing, and $\alpha$ and $\beta$ are the angles of the incident and dispersed rays measured from the grating plane. First and second order spectra are overlapping on the detectors and using the energy resolution of the detector they can be easily separated. For extended sources the wavelength resolution degrades to

$\Delta \lambda=0.124 \times \theta / m$

where $\theta$ is the source extent in $\operatorname{arc} \min$ and $\Delta \lambda$ in $\AA$. The instrument is optimized at $15 \AA$ around the Fe-L lines. Key parameters of the design are listed in Table 1.

Each instrument consists of four different units: the reflection grating assembly (RGA) which is an assembly of accurately positioned gratings; the RGS focal plane camera (RFC) which is an array of 9 CCDs in which the reflected photons are detected; the RGS analog electronics (AE) which contains part of the data processing chain and controls the read-out sequence of the CCDs and the RGS digital electronics (DE) which controls the instrument and performs event pattern recognition.

Reflection gratings (RGA): 182/181 in-plane reflection gratings for RGS1/2 are accurately placed in the exit beam of the X-ray telescopes. Each grating is $200 \mathrm{~mm}$ long by $100 \mathrm{~mm}$ wide with a gold reflecting surface and a ruggedized backside with 5 supporting ribs to control the surface errors. Each grating is mounted at its four corners. To correct for the beam convergence, the line density of the gratings varies with position $x$ on the grating in such a way that line density is 645.6 lines $/ \mathrm{mm}$ at grating center, while being 665.3 lines $/ \mathrm{mm}$ at $x=+100 \mathrm{~mm}$ and 626.8 lines $/ \mathrm{mm}$ at $x=-100 \mathrm{~mm}$. The gratings are mounted in a Rowland circle configuration, on a support structure made of Be because of its mass and low thermal expansion coefficient. To maintain the proper focusing of all gratings the temperature gradient over the grating assembly should be less than $1^{\circ} \mathrm{C}$.

The X-rays are recorded by an array of 9 CCDs in the $R G S$ focal plane camera $(R F C)$. This camera is passively cooled to reduce the dark current to an acceptable level 
$\left(\leq 0.06 \mathrm{e}^{-} / \mathrm{pixel} / \mathrm{s}\right)$. To have a good QE $(\geq 80 \%)$ at low energies, the CCDs are illuminated from the backside, avoiding the absorption that would otherwise be caused by the gate structure at the front side of the CCDs. To reduce the sensitivity for optical load, the CCDs are coated with a thin aluminum layer with a thickness of $45 \mathrm{~nm}, 60 \mathrm{~nm}$ and $70 \mathrm{~nm}$ depending on the relative position of the $\mathrm{CCD}$ with respect to the mirror focus. To isolate the $\mathrm{Al}$ layer from the $\mathrm{CCD}$ itself an insulating $\mathrm{MgF}_{2}$ layer has been applied. These components produce absorption edges in the response of the instrument. Subsequently the X-rays are absorbed in the high-epitaxial Si ( $\geq 10 \Omega \mathrm{m})$ (Jansen et al. 1989). For each absorbed photon the number of electrons is proportional to the energy of the incoming photon. To avoid unnecessary diffusion of the produced electron cloud in the Si prior to charge cloud collection and read out near the gate structure, the CCDs are thin $(30 \mu \mathrm{m})$. This implies that the QE around the Si-edge is less than $100 \%$. In addition, some charge will be lost for events absorbed near the backside of the CCDs. This causes a partial event tail in the CCD redistribution function.

All CCDs have an image and a storage section and are read out in frame transfer mode. The clock pattern for this readout is programmable and it is possible to perform on-chip binning, to read out only parts of the CCD, or to read out the full CCD through one or both of its two output nodes. During standard operations the charge of $3 \times 3$ physical CCD pixels of $27 \mu \mathrm{m} \times 27 \mu \mathrm{m}$ is added on the $\mathrm{CCD}$ (these $3 \times 3$ pixels are referred to as a bin). Still, the charge generated by a single $\mathrm{X}$-ray event can be split over more than one bin causing so-called split events. To ensure the direct relation between charge and photon energy, pile-up (two events in the same bin or in neighboring bins during a read-out) should be avoided. The typical read-out time per CCD, with the two CCD halves read out simultaneously through both output nodes, is $\approx 0.6 \mathrm{~s}$, resulting in a readout time of $4.6 \mathrm{~s}$ for the array of 8 operational CCDs. Except for very bright sources such as e.g. Sco X-1 or the Crab the pile-up is very small (normally well below $1-2 \%$ ).

In addition to X-rays dispersed by the gratings, the cameras also register X-rays from four onboard calibration sources located in the thermal/radiation shield just around the detector. $\alpha$ particles from a $\mathrm{Cm} 244$ source illuminate an Al target generating Al-K line emission in two sources. For the two other sources a Teflon target has been selected, generating F-K $\alpha$ emission. The generated X-rays do not overlap with the image of a point source and their energies do not interfere with the 1 st and 2 nd order dispersed source spectrum.

The analog electronics (AE) controls and selects the CCD to be processed and measures the amount of charge in the output node of the CCD. It amplifies the signal and adds an electronic offset, before passing the signal to the ADC of the digital electronics.

The digital electronics (DE) processes the data. Bins with only the dark current and electronic noise (lower threshold) and bins with a too high charge due to a charged particle hitting the detector (upper threshold) are immediately rejected. The remaining bins are searched for acceptable event patterns (combination of adjacent bins within a $2 \times 2$ bin region which are characteristic for a true $\mathrm{X}$-ray event). Patterns larger than a $2 \times 2$ bin region are rejected. The DE also stores a list of uploaded hot pixels and columns, used for rejection of these items. The relative loss of CCD area due to interactions of charged particles is computed for each CCD readout frame. Later, on ground, the effective exposure time for each CCD readout frame is corrected in correspondence with this relative loss of CCD area and for the known hot pixels and columns.
In addition to this routine data processing, full frames are stored at a very low rate of one every $15 \mathrm{~min}$ and using spare telemetry capacity these full frames are transferred to ground. This provides diagnostic data to monitor the CCD performance in full detail. A cold redundant digital electronic unit is present.

The main observable parameters are the position along the dispersion axis (the photon wavelength), the position in the cross dispersion direction and the photon energy as recorded by the pulse-height in the detector (for order separation). In Fig. 2 these data are shown for a point source. In addition to the X-rays from the celestial source, the four onboard calibration sources with different energies $\left(\mathrm{F}-\mathrm{K}_{\alpha}\right.$ at $0.677 \mathrm{keV}$ and $\mathrm{Al}-\mathrm{K}_{\alpha}$ at $1.487 \mathrm{keV}$ ) are clearly visible.

\section{Calibration basics}

\subsection{Calibration elements}

The response of an instrument describes the conversion from photons incident on the instrument to the data actually recorded by the instrument. For a spectroscopic instrument like RGS the response key elements are effective area, wavelength scale, and linespread function. We define these elements as follows.

Effective area: this quantity is a function of energy $\left(A_{\mathrm{eff}}(E)\right)$, and is defined as the ratio between the observed source count rate (counts s${ }^{-1}$ ) and the incident photon flux (photons $\mathrm{s}^{-1} \mathrm{~cm}^{-2}$ ). For RGS the effective area can be decomposed into the product of the following different components:

$$
\begin{aligned}
& \text { - mirror area } \times \text { mirror efficiency; } \\
& \text { - grating efficiency; } \\
& \text { - CCD quantum efficiency; } \\
& \text { - exposure fraction; } \\
& \text { - data selections. }
\end{aligned}
$$

The different components depend on a multitude of parameters. The mirror geometrical area is the front surface area of the total instrument. The CCD quantum efficiency (QE) includes all losses in the absorbing and dead layers on the CCD, charge transfer efficiency (CTE), onboard signal thresholds and processing etc., and depends on the actual CCD chip and location on the chip. The exposure fraction is relative to the real observing time. Apart from dead times caused by onboard processing limitations and cosmic ray hits it also includes the effects of instrument pointing variations. These variations move photons to different pixels on chip locations with different QE or dead pixels. Subsequent data selections also affect the number of recorded photons.

Linespread function $(L S F)$ : this is the probability distribution of the location where photons of given energy land on the detector, $\operatorname{LSF}(E$, pointing, ..). This depends on the quality and internal alignment of the optics, both in terms of optical surface accuracy and scattering contamination. Pointing reconstruction accuracy and data selections also contribute.

Wavelength scale: this function is defined as the relation between the energy of the incoming photon and the most likely location, which is the center of the linespread function, where it will end up on the detector, $W(E)$. This depends on pointing, grating parameters and the internal alignment of the different instrument components.

Table 2 gives an overview of the different dependencies. In the next sections these components are treated in more detail.

All response elements may change over time, but during the operational period a few major adjustments caused sudden 

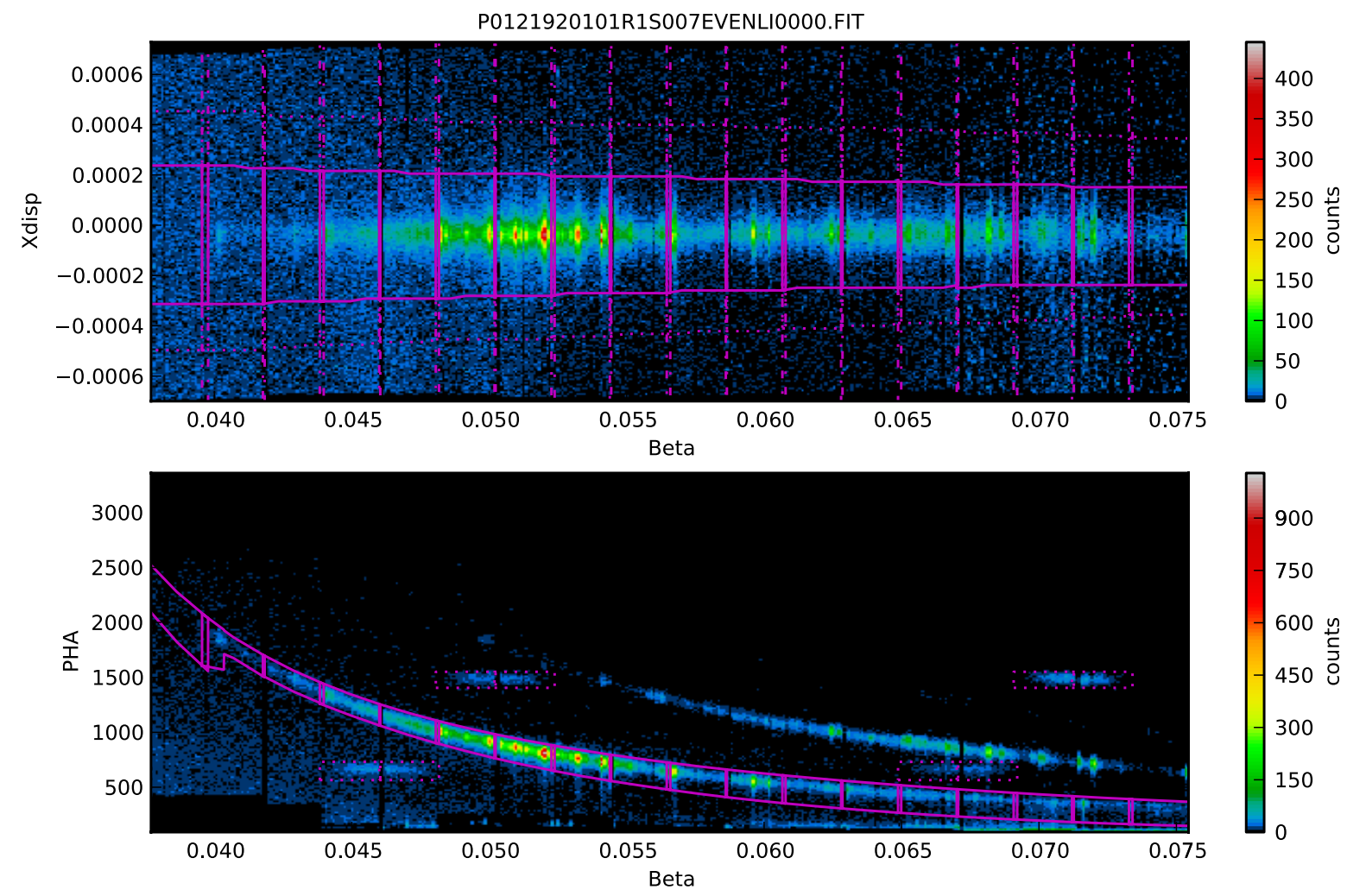

Fig. 2. Capella observation (id: 0121500201001). The color scale represents a logarithmic intensity scale. The dispersion (Beta) axis runs horizontally and increases to the right. All angles (Beta, Xdisp) are in radians. In the top panel the cross dispersion (Xdisp) is along the vertical axis whereas in the bottom panel this is replaced by the CCD recorded photon energy, or PHA, in units of eV. In the bottom panel the low level thresholds are clearly visible. Standard data selections for first order are indicated by the solid purple curves.

Table 2. Calibration dependency.

\begin{tabular}{|c|c|}
\hline Parameter & Dependency \\
\hline Linespread function & $\begin{array}{l}\text { mirror PSF } \\
\text { gratings alignment } \\
\text { gratings scattering } \\
\text { particle contamination of the optics } \\
\quad \text { (causes additional scattering) } \\
\text { pointing reconstruction accuracy }\end{array}$ \\
\hline Wavelength scale & $\begin{array}{l}\text { grating dispersion parameters } \\
\text { mirror - grating assembly alignment } \\
\text { focal plane camera position } \\
\text { CCD positions in the camera } \\
\text { stability of optical bench } \\
\text { pointing reconstruction accuracy }\end{array}$ \\
\hline Effective area & $\begin{array}{l}\text { mirror effective area } \\
\text { geometrical overlap gratings/mirrors } \\
\text { grating efficiency } \\
\text { CCD quantum efficiency } \\
\text { CCD molecular contamination } \\
\text { CCD "absorption" layers (filters) } \\
\text { CCD thickness } \\
\text { event pattern processing } \\
\text { CCD redistribution } \\
\text { read-out noise } \\
\text { CCD dark current } \\
\text { charge transfer inefficiency } \\
\text { X-ray event pattern } \\
\text { exposure } \\
\text { satellite pointing } \\
\text { CCD cosmetic defects } \\
\text { particle background } \\
\text { data selections }\end{array}$ \\
\hline
\end{tabular}

changes to specific components. Further cooling of the detector, change of CCD readout mode and adjustment of CCD bias voltage had major impact on the response. Details of these instrumental operations are described in Sect. 11 and listed in Table 12 .

\subsection{Calibration method}

Accurate calibration is a long and tedious process. As a full mapping of the response over all energies and all source positions is impossible, we combine models, constrained or verified by real measurements. Subsequently this model was updated using inorbit data.

The calibration starts with the physical understanding and measurement of the response for different units including the mirror, the detector (first as CCD, subsequently as integrated camera) and the reflection grating assembly (first as grating plate, later as integrated RGA).

Following the detailed calibration of the physical models, the instrument response is compared with end-to-end performance tests at the MPE Panter facility (Freyberg et al. 2005; Bräuninger et al. 2004) at specific energies and positions in the field of view. The major limitations of this approach are:

- the measurements can be performed only for a limited number of energies and angles;

- no X-ray sources with narrow emission lines below $\sim 1 \mathrm{keV}$ exist;

- due to the finite source distance the first part of the mirror is not fully illuminated resulting in a not fully representative measured mirror response; 
- the alignment of the units cannot be tested on the ground as the correction for the finite source position $(120 \mathrm{~m})$ requires a different relative alignment of the units than in orbit.

To cope with these limitations for the ground calibration a dedicated calibration campaign in the first months in orbit was foreseen. With a number of astrophysical sources unknown calibration parameters, such as the wavelength scale, could be fixed.

Following this early phase in-orbit calibration a set of "routine" calibrations are performed with different aims:

- Effective area stability: the stability of the effective area is checked by bi-annual measurements of two stable soft sources: the isolated neutron star RX J1856-3754 and the Vela Pulsar Wind Nebula. Two other sources turned out to be less suited: the isolated neutron star RX J0720.4-3125 showed to be variable (de Vries et al. 2004), and supernova remnant 1E0102.2-7219 (or N132D) is an extended source. In extended sources, spatial and spectral information is mixed, which means that if the source is not circularly symmetric, the extracted spectrum depends on the roll angle of the satellite, and is therefore less suitable for a regular verification of the effective area. Measurements of a strong continuum source (BL Lacs PKS 2155-304 and Mrk 421) allow a check on the stability of the oxygen edge, which can change over the mission lifetime due to the accumulation of ice on the cooled detector.

- Wavelength scale: initially a set of coronal sources were measured regularly. AB Dor once a year, Capella and HR 1099 alternating also once a year. Recently this has been reduced to Capella once a year only because the wavelength scale does not show long term trends.

- CCD response: dedicated measurements with Mrk 421 on and off-axis allows the measurement of the CTI in the detectors. For different positions off-axis in the cross-dispersion direction, the number of parallel transfers in the CCD varies, and this allows an accurate calibration of the parallel CTI. The sides of two adjacent CCDs are read out in opposite direction. For RGS2, after revolution 1408, all pixels are read out trough one single node. Due to these serial transfers the pixels near the CCD edges are hardly affected whereas the pixels in the center, with typically 512 or 1024 transfers, show a reduction of a few percent in pulse height. This is observed as a saw tooth shape of the recorded CCD pulse heights versus dispersion direction in the spectrum, that disappears after calibrating the serial CTI.

In addition, the observations of Capella are used to verify the CCD electronic readout gain, defined as the conversion factor from captured electric charge to pulse height readout units, and the serial CTI calibration of the CCDs.

Furthermore, full frame CCD images, obtained during all observations, allow us to monitor the development of artifacts due to radiation damage in the CCDs and the changes in the read-out noise and dark current of the CCDs.

- Non-routine calibrations: in addition, non-routine calibrations are carried out to investigate certain aspects. A detailed measurement of the Crab spectrum was carried out with the aim of calibrating the absolute effective area (Kaastra et al. 2009) and of Sco X-1 with the aim to fully characterize the $\mathrm{X}$-ray absorption edge structures present in the CCDs response (de Vries et al. 2003).

Some of these measurements were planned to coincide with the same target in the Chandra observatory observation program. Detailed comparison of our results with those of Chandra is, however, outside the scope of the current paper. Information on cross calibrations between high energy missions can e.g. be found in the proceedings of the IACHEC meetings ${ }^{1}$.

\subsection{Calibration files}

The basic tool to analyze RGS spectra is the instrument response matrix (RSP). The response matrix is the product of the monoenergetic X-ray redistribution (RMF), the effective area (ARF) and exposure map. Data channels are re-binned CCD columns in the dispersion axis $(\beta$-angle) or in wavelength $(\lambda)$ space. The exposure map contains for each data channel the relative exposure time of that channel with respect to the exposure time of the observation. E.g. discarded bad CCD pixels or columns in the instrument, CCD dead times due to lost telemetry packets are accounted for in the exposure map. In the total response matrix, all these different elements of the calibration are combined.

Over time the understanding of the instrument response will evolve, both in terms of additional or improved calibration data, and in terms of improved instrument models. In addition, the calibration of the instrument is not static but varies over time. This is accommodated by the current calibration files (CCF). These CCFs store the parametrized instrument response and are used by the instrument model. The CCFs are tagged with a time validity interval, usually spanning many observations, which allows to have parameters which slowly change with time (gain, CTI, bad pixels). Changes can be accommodated by updating the relevant CCF. Only occasionally the actual data processing code needs to be updated to accommodate improvements in instrument models. The relevant CCFs are given in Table 4. The actual CCFs, including their sequence number and validity start time are given in Table A.1. The CCFs were designed with flexibility in mind; some CCFs contain elements which later turn out not to be used.

Main steps in the RGS data calibration processing are as follows.

- Event processing: from each recorded event, the CCD noise level is subtracted on a pixel by pixel basis, as derived from a three orbit average computed from CCD images, and the recorded pulse level (PHA) is converted to $\mathrm{eV}$ using the gain and CTI calibration. Warm/hot pixels are detected, and corresponding events discarded.

- Wavelength: using the spacecraft pointing information and instrument geometry, the conversion from detector coordinates to $\beta /$ wavelength space is computed.

- Spectra: using the given spatial, PHA, and time selections within the observation, source and background spectra are computed in detector channel (either $\lambda$ or $\beta$ space).

- Response: using the appropriate calibrations for the time of observation, the response matrix is computed.

Instrumental background is usually obtained from off-source regions in cross dispersion on the detectors, using identical PHA selections as for the source. This works very well for sources which illuminate only a fraction of the RGS crossdispersion field of view (4.9 arcmin), but cannot be used for extended sources which cover the entire detector. For this reason a separate set of calibration files is maintained which provide backgrounds derived from empty fields. These backgrounds are mainly generated by the fluctuating soft proton flux and cover a variety of different flux levels. The background in this case is constructed by selecting the appropriate background calibration

http://web.mit.edu/iachec/ 
Table 3. Current inflight calibration plan.

\begin{tabular}{lcl}
\hline \hline Purpose & Frequency (/year) & Measurement \\
\hline$\lambda$ calibration & 1 & coronal source: Capella \\
Effective area & 2 & continuum source: Mrk 421, PKS 2155-304 \\
& 1 & Vela supernova remnant \\
& 1 & RX J1856-3754 \\
CCD response (gain/CTI) & 0.5 & Mrk 421 on and off axis (CTI) ${ }^{1}$ \\
& 1 & Capella (gain) \\
\hline
\end{tabular}

Notes. ${ }^{(1)}$ During the period when Mrk 421 is not visible, it is replaced by PKS 2155-304.

Table 4. CCF files, applicable to RGS.

\begin{tabular}{|c|c|}
\hline $\mathrm{CCF}$ & Contents \\
\hline ADUConv & $\begin{array}{l}\text { gain (ADC to eV conversion) } \\
\text { average noise level (not used) }\end{array}$ \\
\hline CTI & parallel and serial CTI correction \\
\hline BadPix & $\begin{array}{l}\text { bad pixels, segments and columns } \\
\mathrm{h}=\text { uploaded, } \mathrm{H}=\text { on ground only }\end{array}$ \\
\hline CoolPix & additional hot/cool pixels \\
\hline Quantum Efficiency & $\begin{array}{l}\text { CCD filter layers and Si thickness } \\
\text { RGA efficiencies and vignetting }{ }^{1}\end{array}$ \\
\hline EXAFS & $\mathrm{MgF}_{2}$ edge and $\mathrm{O}$ edge \\
\hline EffAreaCor & $\begin{array}{l}\text { contamination correction } \\
\text { correction for large scale variation }\end{array}$ \\
\hline ReDist & CCD PHA redistribution parameters \\
\hline LineSpreadFunc & $\begin{array}{l}\text { RGA figure/alignment errors and scat- } \\
\text { tering parameters }\end{array}$ \\
\hline CrossPSF & source profile in cross dispersion \\
\hline LinCoord & $\begin{array}{l}\text { relevant alignment parameters affecting } \\
\text { wavelength scale }\end{array}$ \\
\hline TemplateBackground & $\begin{array}{l}\text { spectra for pre-defined background } \\
\text { intensities for data selections }\end{array}$ \\
\hline XRT XAreaEff & mirror effective area and vignetting ${ }^{2}$ \\
\hline XRT XPSF & $\begin{array}{l}\text { mirror RGS point source PSF profile, } \\
\text { used for LSF computation }\end{array}$ \\
\hline XMM BoreSight & instrument alignment parameters \\
\hline MiscData & various additional parameters \\
\hline HKParmInt & RGS housekeeping parameter limits \\
\hline SAACorr & $\begin{array}{l}\text { coefficients for the sun angle wave- } \\
\text { length correction }\end{array}$ \\
\hline ClockPatterns & CCD clock pattern parameters \\
\hline XMM ABSCoef & $\begin{array}{l}\text { Henke absorption coefficients for cer- } \\
\text { tain elements }\end{array}$ \\
\hline
\end{tabular}

Notes. ${ }^{(1)}$ The geometrical effect that for photons leaving a grating at larger angles the backside of the neighbouring grating may block part of the X-rays (shadow effect). This depends on spacecraft pointing and energy of the photons. ${ }^{(2)}$ The geometrical effect that for off-axis angles, the mirror shells may partially block each other. Blocking is different for different parts of the mirror, which means there will be some energy dependence, since reflection efficiency differs depending on photon energy and reflection angle.

files, based on the soft proton level indicators constructed from CCD9 count rates (see also Sect. 10.1).

\section{Mirror calibration}

The two main functions describing the mirror response are (a) the point spread function and (b) the effective area. Of course they depend on the offset from the optical axis. In Fig. 3 we show the measured mirror PSF, projected on the RGS dispersion axis, and the mirror model used for RGS. The model consists of

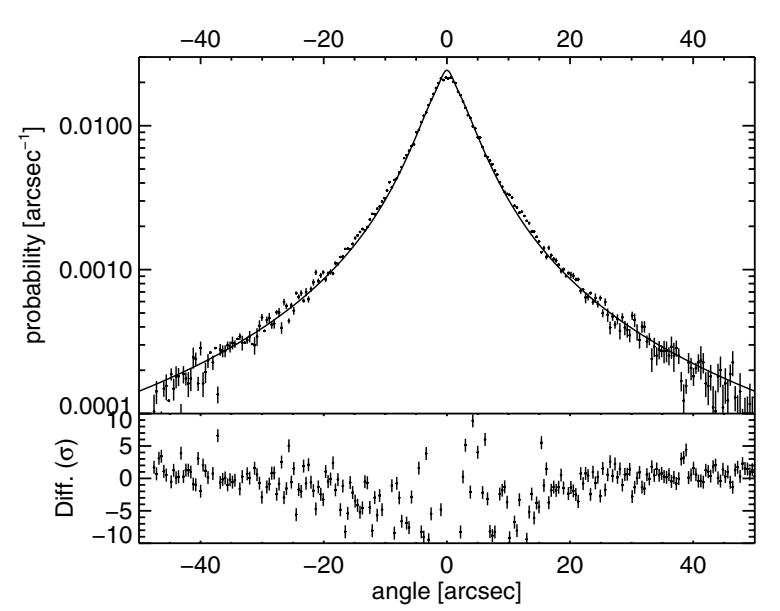

Fig. 3. Measured and modeled mirror response (projected on the dispersion axis). Fit residues (lower plot) are given in units of the statistical error.

a combination of a Gaussian and a Lorentzian function, which are parametrized in the CCF.

Although systematic effects dominate there is a good match, in general within $2-4 \sigma$ for the wings, between the model and the data with the exception of an overprediction towards the core of the distribution. This can be explained by some pile-up in the core of the measured distribution. However, the angular scale of this mismatch is small compared to the RGS LSF, so this hardly affects the modeled LSF.

The total RGS LSF width $(\approx 30$ arcsec FWHM) combines the mirror PSF $(\approx 6$ arcsec FWHM) and the grating scattering and internal misalignment's. RGA scattering is the most dominant factor (see Fig. 28).

\section{Detector calibration}

Due to radiation damage, the detector properties change over time.

The CCD response is only used to separate orders and has only an indirect effect on the spectral resolution for a grating spectrometer. Its main impact is the data selections in CCD pulse height that affect the instrument effective area.

\subsection{Detector model}

We developed a model for the detector response based on a simplified and phenomenological approximation of the interaction of a photon and the detector. Figure 4 shows a crosscut through an RGS back illuminated CCD. The probability $P$ for absorption of the X-ray photon at penetration depth $x$ is:

$\frac{\mathrm{d} P}{\mathrm{~d} x}=\frac{1}{\tau} \cdot \mathrm{e}^{-x / \tau}$ 


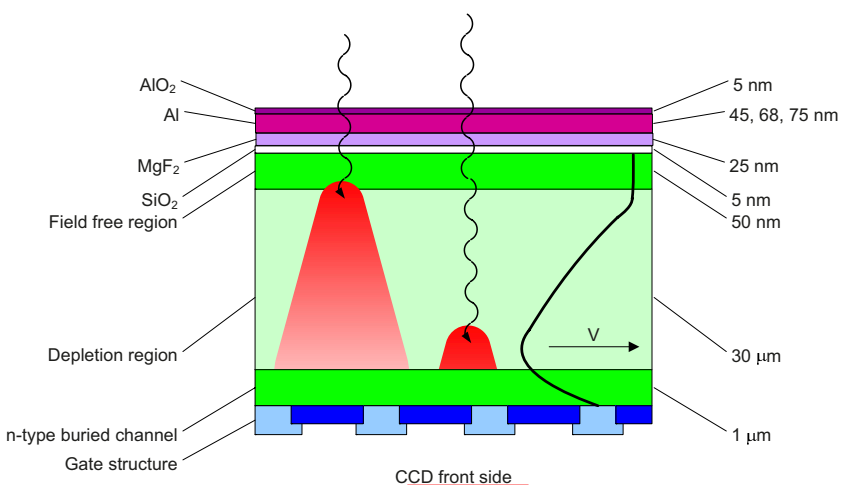

Fig. 4. Model of the CCD indicating all different layers. X-rays entering from the top will be absorbed at a certain depth. An X-ray photon of low energy might be absorbed close to the field free region, losing part of its charge; higher energy X-rays will be absorbed closer to the gate structure and therefore the charge diffusion in the depleted region will be smaller (indicated by the flat mountain shaped area in the figure). At the right the potential in the CCD is indicated.

with $\tau(E)$ the absorption depth for silicon for X-ray photon energy $E$ as derived from Henke et al. (1982).

At the spot of the X-ray photon absorption, an amount of electric charge is released proportional to the incident energy of the photon. We define the electric charge in units of the energy of the X-ray photon. This scale of course implies the calibration of the electronic signal.

Not all charge released will make it to the output node of the CCD. The collected charge $Q$ from the X-ray event depends on the depth at which the charge is generated. The deeper the penetration, the closer to the front side structure and the more of the generated charge is collected. This is modeled by:

$Q= \begin{cases}0 & \text { for } E<T \\ T+(E-T) \cdot\left(1-\mathrm{e}^{-(x / b)^{1 / 3}}\right) & \text { for } E \geq T\end{cases}$

with $T$ the threshold for charge detection and $b$ a scale parameter. These parameters were derived from ground calibrations and set at $T=50 \mathrm{eV}$ and $b=20.95 \mathrm{~nm}$.

The charge probability density can be written as:

$\frac{\mathrm{d} P}{\mathrm{~d} Q}=\frac{\mathrm{d} P}{\mathrm{~d} x} \cdot \frac{\mathrm{d} x}{\mathrm{~d} Q}$.

From (4) we derive:

$\mathrm{d} Q=(E-T) \cdot \mathrm{e}^{-(x / b)^{1 / 3}} \cdot\left(\frac{x}{b}\right)^{-2 / 3} \cdot \frac{1}{3 b} \mathrm{~d} x$

and:

$\frac{\mathrm{d} P}{\mathrm{~d} Q}=\frac{1}{\tau} \cdot \mathrm{e}^{-x / \tau} \cdot \frac{3 b}{E-T} \cdot \mathrm{e}^{(x / b)^{1 / 3}} \cdot\left(\frac{x}{b}\right)^{2 / 3}$.

From (4) it follows:

$\left(\frac{x}{b}\right)^{1 / 3}=\ln \frac{E-T}{E-Q}$

which allows to eliminate $x$ from (7):

$\frac{\mathrm{d} P}{\mathrm{~d} Q}=\frac{3 b}{\tau} \cdot \frac{1}{E-Q} \cdot \ln ^{2}\left(\frac{E-T}{E-Q}\right) \cdot \mathrm{e}^{-\frac{b}{\tau} \ln ^{3}\left(\frac{E-T}{E-Q}\right)}$.

To this function (9) a constant partial event floor $t$ is added that has constant probability density below the incident energy

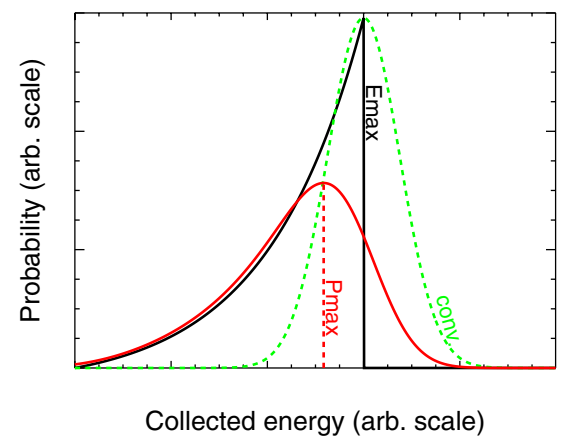

Fig. 5. Schematic view of the effect of convolving the strongly asymmetric collected charge probability (solid black curve) for a photon with energy $E_{\max }$ with a symmetric Gaussian shape (broken green line) of the noise distribution. The resulting probability distribution (red curve) will have its maximum $P_{\max }$ shifted to lower energy than the original photon energy $E_{\max }$.

and is zero above. The resulting redistribution function $\frac{\mathrm{d} R}{\mathrm{~d} Q}$ thus becomes:

$\frac{\mathrm{d} R}{\mathrm{~d} Q}= \begin{cases}\frac{\mathrm{d} P}{\mathrm{~d} Q} & \text { for } Q \geq E \\ \frac{\mathrm{d} P}{\mathrm{~d} Q}+t & \text { for } Q<E .\end{cases}$

The partial event floor $t$ is normalized to the total partial event fraction $P_{\text {part }}$ :

$P_{\mathrm{part}}=\int_{0}^{E} t \mathrm{~d} Q=t \cdot E$.

$P_{\text {part }}$ is 0.0348 for $E=1.9 \mathrm{keV}$ and scales with $\left(1-\mathrm{e}^{-1.832 \cdot E}\right) \times$ $\tau(1.9 \mathrm{keV}) / \tau(E)$ with $E$ expressed in $\mathrm{keV}$.

Finally, this shape is convolved with a Gaussian with a width of $\sigma$ to account for the Fano (Fano 1947) and amplifier noise:

$\sigma=\sqrt{(a \cdot \sqrt{s} \cdot w)^{2}+w f E}$

with $a=5.4$ the amplifier noise, $w=3.65$ the electron creation energy, $f=0.14$ the Fano noise factor and $s$ a scale factor, depending on the OCB pixel size with $s=1.4$ for $3 \times 3 \mathrm{OCB}$ and $s=2.5$ for $1 \times 1$ OCB.

Figure 6 shows the different components in the final model function. This CCD redistribution function is checked in flight at regular intervals to see whether the CCD charge collection is stable using bright mono-energetic emission lines of our wavelength calibration sources (notably Capella) (Figs. 7 and 8). The incomplete charge collection of charge created near the back side (see Fig. 4) which mainly affects low energy X-rays, or X-rays just above the $\mathrm{Si}$ edge, causes the tail of the function to lower energies. This strongly asymmetric function with its tail towards low energies, combined with the convolution by a symmetric Gaussian for the Fano and amplifier noise, causes the peak of the function to be shifted towards lower energies, as schematically shown in Fig. 5, to below the original incident X-ray energy. The effect is less at higher energies. In both Figs. 7 and 8 the effect of leakage of the second order events is shown. Also clearly visible is the improved resolution at higher energies and the tail of the noise floor at lower energies.

This redistribution function can be regarded as the response to mono-energetic incident X-rays, in which the peak of the collected energy does not necessarily coincide with the initially absorbed energy. Using such a distribution enables calibrating a simple linear gain relation between collected charge 


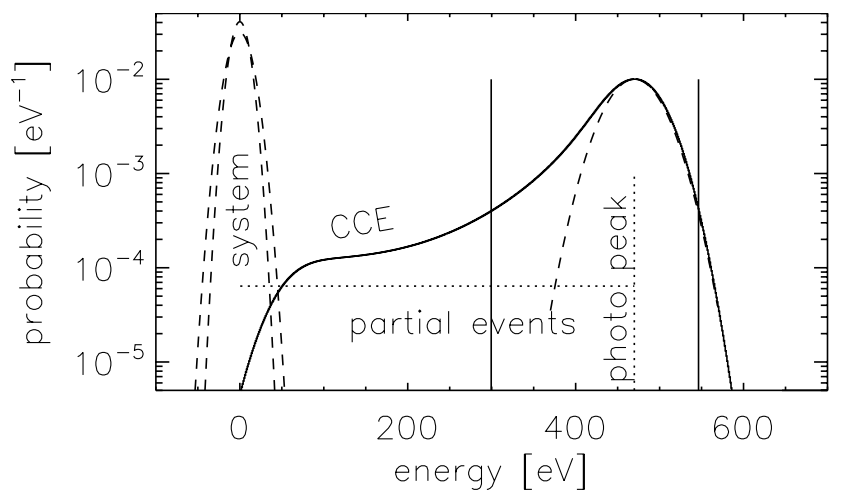

Fig. 6. Model of the CCD response, or charge collection efficiency (CCE), which is the probability for the collected energy, illustrating the key components. Also shown is the $95 \%$ window which is the default data selection (vertical lines). For completeness we also show the typical read-out noise (system) which gives the typical output for a pixel without absorbed X-ray. The electronic bias level of the CCD has been subtracted from the energy axis, causing the read-out noise system peak to extend below the zero energy level.

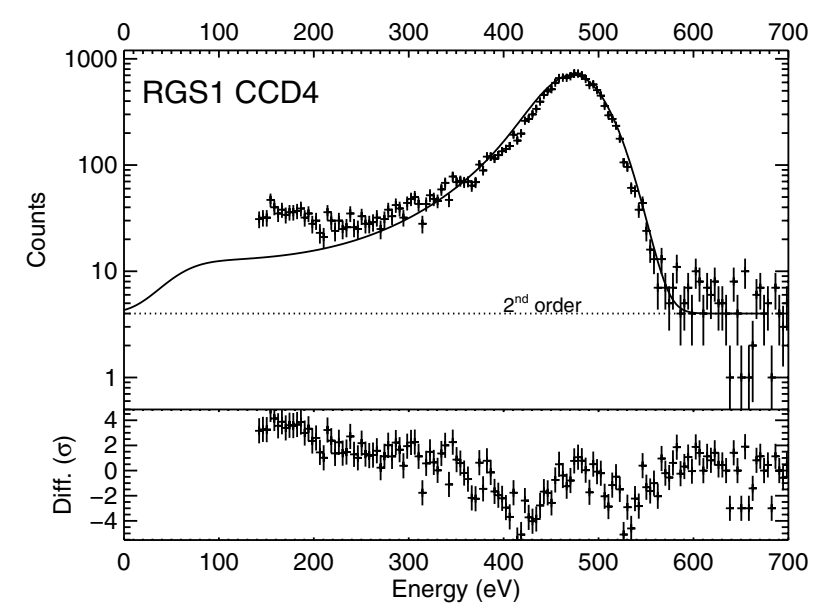

Fig. 7. Modeled and observed CCD response for the N VII Ly $\alpha$ line $(24.779 \AA=500 \mathrm{eV})$. The data is a sum of 15 Capella measurements.

and CCD electronic output. When the observed peak of the redistribution function would be used to define the CCD energy scale, a highly non-linear function would be needed.

The user can define any window for event selection. Generically the window is defined in terms of percentage of the integrated CCD redistribution function at equal cutoff levels left and right of the maximum. The default selection uses $95 \%$ of the events, identical for all energies. Figure 6 shows this selection. This nominal window is relatively insensitive to small variations in CCD gain and uncertainty in the redistribution model. The CCD gain is calibrated at two yearly intervals by shifting the observed CCD redistribution to the model distribution. The uncertainty in the gain calibration is estimated at $\leq 1 \%$. The effect of the gain uncertainty on effective area is far less than $1 \%$, since this is a second order effect.

Whereas the number of selected events, which are the actually detected photons, is largely insensitive to gain variations, the effect of the visible/UV blocking filters as well as variations in the Si thickness are clearly important. Si thickness variations over the CCDs were measured by using the interference patterns of internal reflections of infrared light $(1032 \mathrm{~nm})$ within the CCD chip. Due to the production process the CCDs are thinner near the edges whereas the central part of each CCD is typically

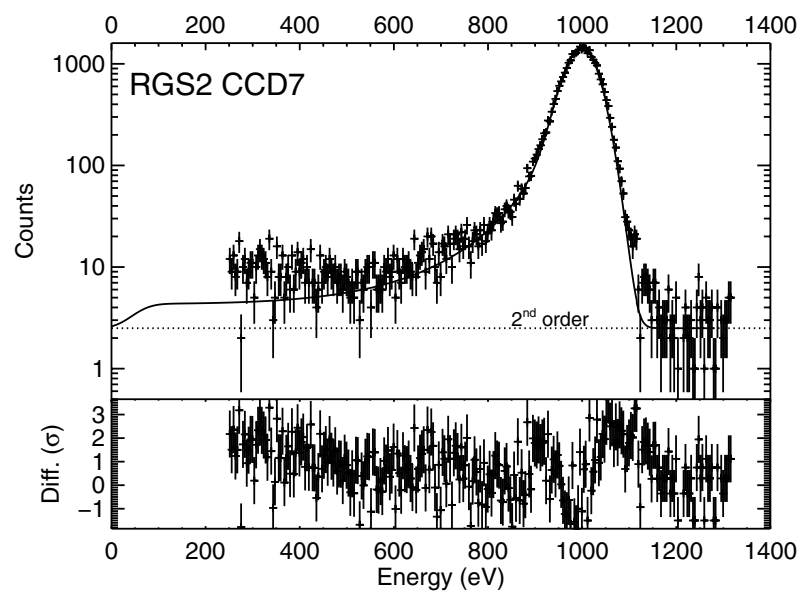

Fig. 8. Modeled and observed CCD response for Ne X Ly- $\alpha$ line $(12.132 \AA=1022 \mathrm{eV})$. The data is the sum of 15 Capella measurements.

flat within $2 \mu \mathrm{m}$. The edges can be up to 3 (one side) or up to $6 \mu \mathrm{m}$ (other side) thinner which effects the QE at $1.8 \mathrm{keV}$ of at most $-4 \%$. This effect is only relevant for those CCDs which cover the wavelength ranges between 1.5 and $1.82 \mathrm{keV}$ (Si-edge) and is properly taken into account for the relevant CCDs in the calculated efficiencies.

In the model we have included absorption by the Al filter, deposited on top of the CCD with a varying thickness between 44 and $75 \mathrm{~nm}$ and a $\mathrm{MgF}_{2}$ isolation layer between 23 and $29 \mathrm{~nm}$ located in between the $\mathrm{Al}$ layer and the $\mathrm{Si}$ (see Fig. 4), as measured by the CCD manufacturer (EEV). The uniformity of these layers was verified by UV/optical measurements at 250, 406 and $540 \mathrm{~nm}$. Interpretation of these results was hampered by the lack of knowledge about the surface roughness of these layers. The effect on total absorption of possible variations in surface roughness below $24 \AA$ is estimated to be less than $1 \%$ increasing to up to $9 \%$ at the longest wavelength $(>35 \AA)$.

All these factors, which also include the signal selection thresholds, are taken into account to determine the total QE of the CCD and these results were verified by ground measurements (Fig. 9). The shown model is the calculated QE based on physical (and measured) properties of the device as given in Table 5. A fair match between the measured and calculated QE is observed but, particularly at lower energies, for the C, O and $\mathrm{F}$ sources large deviations could easily occur. This is mostly due to the fact that at low energy the natural line width of these sources is relatively large. Attempts were made to use a separate monochromator to narrow the natural line widths of the sources, but these results turned out to be less reliable due to unpredictable and uncalibrated inhomogeneities in the monochromator beam.

\section{2. $C C D$ radiation hardness}

Cosmic charged particles hitting the CCD detectors can damage the CCD. Either the Si depletion region is damaged, giving rise to local low resistivity causing high dark current and hot pixels, or the charge transfer layer at the front side (opposite the back side where the X-ray photons enter) is hit, which may cause an increase in charge transfer inefficiency (CTI). Increased CTI will cause a drop in the PHA pulse height and a decrease in the CCD energy resolution. When CTI becomes too high, 1st and 2 nd orders cannot be separated any more and the dispersed spectrum will become indiscernible from the system peak. 
Table 5. CCD parameters ranges.

\begin{tabular}{lll}
\hline \hline Al thickness CCD [nm] & $44-75$ & depends on CCD position \\
$\mathrm{MgF}_{2}$ thickness [nm] & $23-29$ & different per CCD \\
Event size $(3 \times 3 \mathrm{OCB})$ & 1.4 & binned pixels \\
Individual CCD total central Si thickness $[\mu \mathrm{m}]$ & $26.6-33.4$ & different per CCD \\
Si change of thickness over single CCDs $[\mu \mathrm{m}]$ & $3-6$ & decrease of thickness towards CCD edges \\
\hline
\end{tabular}

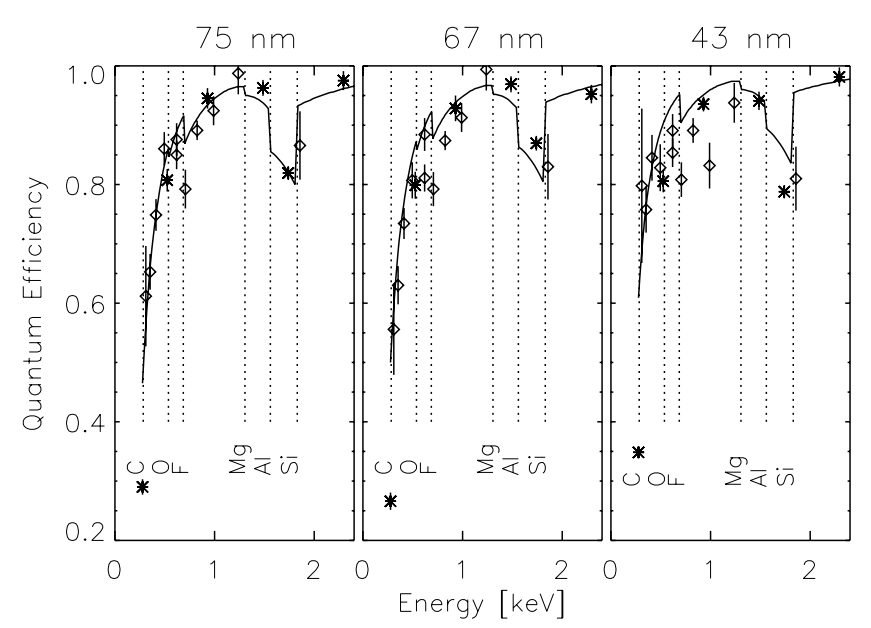

Fig. 9. CCD quantum efficiencies, as measured during ground calibrations at Panter (den Herder et al. 2001). The symbols indicate the measured data points. Stars: X-ray atomic line energies, diamonds: monochromator data. The broken lines indicate the energies of atomic edges of the indicated atomic species. The solid line is our model.

A set of protection measures have been implemented. By applying proper shielding around the detector, $3 \mathrm{~cm} \mathrm{Al} \mathrm{coated}$ with a Au layer, only particles with high energies, mostly protons, will reach the detector directly in addition to the secondary radiation from these particles in the shielding. Also the design of the CCDs and their operations reduce the effect of radiation damage. Buried channels in the CCDs confine the charge to a limited volume, reducing the relative size of the damage with respect to the signal. An operational temperature range can be selected for which the dark current and cosmetic blemishes can be reduced. Together these measures allow to handle the expected dose of $10^{9} 10 \mathrm{MeV}$ equivalent protons and $1 \mathrm{krad}$ (after applying the $3 \mathrm{~cm}$ shield), which is representative for the expected dose over a mission of 10 year (Jansen et al. 1989).

However, Chandra experienced an unexpected damage of their CCDs during the first part of the mission (Prigozhin et al. 2000; O'Dell et al. 2000). This could be attributed to soft protons focused by the X-ray mirrors onto the detectors, encountered during the passage through the radiation belts. To quantify this effect for our detector, flight representative RGS CCDs were subjected to protons at various low energies in the Van der Graaff accelerator in Utrecht. Table 6 presents the results for one representative CCD illuminated from the CCD backside, which is the same side as illuminated in orbit. The device was about $24 \mu \mathrm{m}$ thick although there is a significant variation over the CCD. We selected 6 different energies with a stopping range from $10 \mu \mathrm{m}$ to $30 \mu \mathrm{m}$. At low energies the protons are stopped in the bulk material whereas at the higher energies the protons are stopped in the layer where the charge is transferred. At the highest energies the majority of the protons are stopped by the gate structure, or pass the CCD without causing serious damage.

In Fig. 10 the change in CTI is given for different radiation levels and different proton energies. Although it was planned

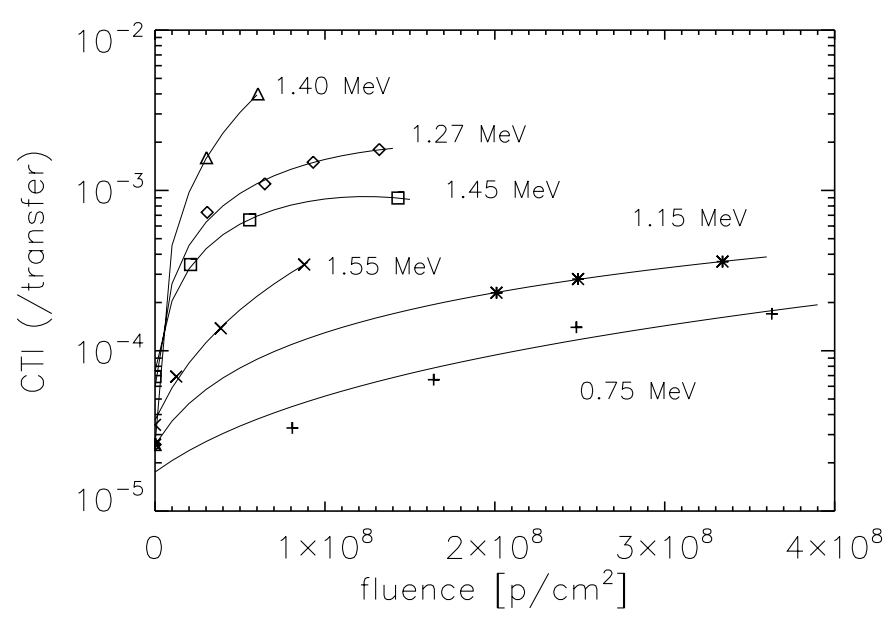

Fig. 10. CTI as a function of the fluence for different energies of the protons. Curves are fit to the data.

Table 6. Sensitivity of RGS CCDs for soft protons normalized to a fluence of $5 \times 10^{7}$ protons per $\mathrm{cm}^{2}$.

\begin{tabular}{lrrr}
\hline \hline$E_{\text {proton }}$ & Stopping range & CTI/transfer & $\begin{array}{l}\mathrm{I}_{\text {dark }} \\
\mathrm{MeV}\end{array}$ \\
$\mu \mathrm{m}$ & {$\left[10^{-5}\right]$} & ratio (post/pre) \\
\hline 0.75 & 10.9 & 3.4 & 6 \\
1.15 & 20.5 & 7.8 & 2 \\
1.27 & 23.8 & 95.7 & 5 \\
1.40 & 27.7 & 306.6 & 46 \\
1.45 & 29.2 & 61.6 & $\mathrm{n} / \mathrm{a}$ \\
1.55 & 32.4 & 18.0 & $\mathrm{n} / \mathrm{a}$ \\
\hline
\end{tabular}

Notes. Stopping ranges from SRIM: http://wWw. SRIM.org

to test each energy up to a fluence of $10^{8}$ protons $/ \mathrm{cm}^{2}$ at the lower energies the beam was about a factor of two more focused than anticipated. This explains the differences in the maximum fluences. As can be seen the CTI degrades most between 1.27 and $1.40 \mathrm{MeV}$, corresponding to a stopping range between 24 and $32 \mu \mathrm{m}$, which is in fair agreement with the thickness and uniformity of the CCD. In addition, we observed that the charge transfer efficiency improves considerably if the CCD is stored at a higher temperature for one night following the irradiation by the protons. This is a known effect in CCDs. In Fig. 11 we provide the integrated CTI (over typically 200 transfers) for 4 different energies at their maximum fluence. As is also known from higher energy protons, the largest CTI occurs between -80 and $-100{ }^{\circ} \mathrm{C}$. At higher temperatures the CTI improves artificially; due to the high dark current the traps are filled for a large fraction of the time. However, under this condition it is hard to impossible to separate the real events from the dark current. Cooling the CCDs below $-100^{\circ} \mathrm{C}$ improves the CTI considerably because the release time of charge from the traps becomes longer and thus their impact on the CTI will be less (they are filled for an increasing part of the time). The curves in Fig. 11 are for a different total dose. No data were measured to correct for 


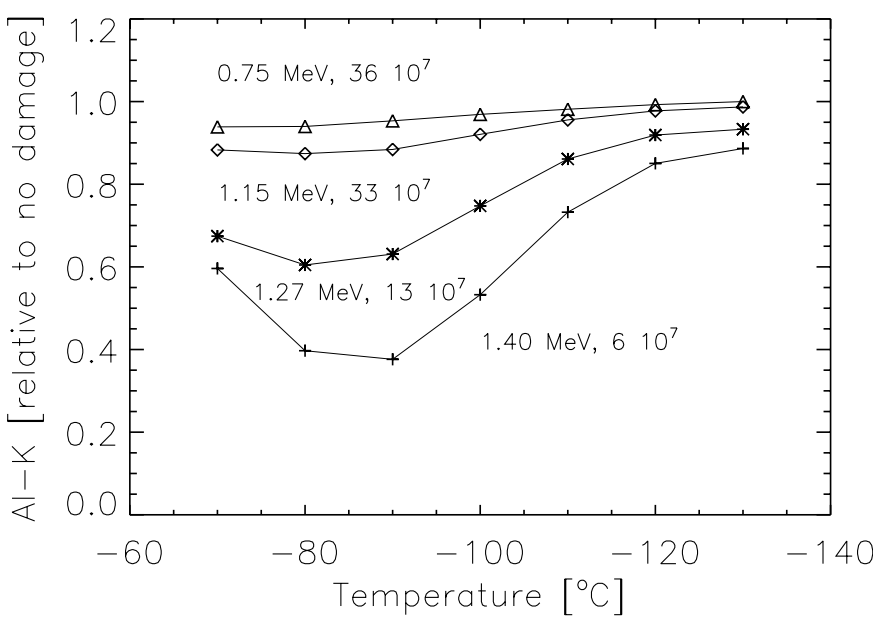

Fig. 11. CTI as reflected in the PHA position of the Al-K line as function of temperature and radiation dose.

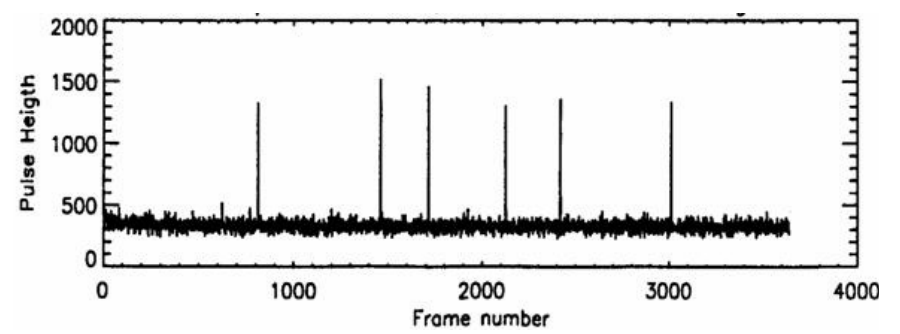

Fig. 12. Response of a single detector pixel to X-rays.

this at other temperatures than $-80^{\circ} \mathrm{C}$. Finally we list in Table 6 the increase in the dark current. This also peaks at $1.40 \mathrm{MeV}$ which is consistent with stopping of the protons around the interface between the bulk Silicon and the gate structure.

Based on these results, the energy dependence of the proton scattering from the mirror and gratings (Sect. 6.4), and the spectral shape for soft protons $\left(E^{-1.7}\right)$, the RGS CCDs were expected to be hardly affected by soft protons. This has indeed been confirmed, although it is impossible to separate soft and hard protons using flight data only. The absence of a filter wheel has not caused any unexpected problems. To identify a time period of high proton flux the RGS detector closest to the optical axis (CCD9) is monitored and if the count rate for a part of the CCD not corresponding to the source image increases above a set level, the EPIC filter wheel will be moved to its closed position.

\subsection{Read-out noise and dark current}

In Fig. 12 we show the typical response of a single detector bin when exposed to X-rays. Most of the time no X-rays are observed, and the detected charge is a measure for the electronic noise and dark current. When an X-ray is absorbed in a bin the corresponding charge increases (the spikes in the figure). The data free from X-ray events are called the noise floor or system peak in the RGS specific descriptions.

The noise floor level is formed by the integrated dark current, on top of the electronic offset. Due to radiation damage and operational conditions, dark current and consequently the noise floor gradually changes over the mission lifetime (Fig. 13). Major changes in the operational conditions are indicated. Clearly the increase due to a strong solar flare (orbit 110), the change due to the CCD bias tuning (orbit 168), the reduction in dark current

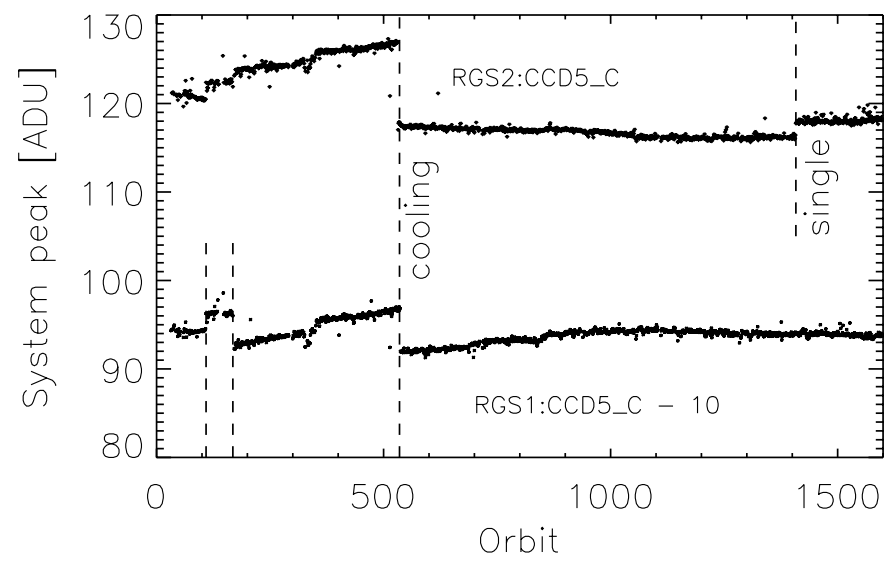

Fig. 13. Evolution of system peak for one node of CCD5 in RGS1 and in RGS2 (other CCDs show a similar behavior). The sudden increase of the peak around orbit 110 (most prominent in RGS1) is due to a solar flare, while the drop around orbit 168 (also indicated for RGS1) is due to the change in bias setting. Cooling lowers the system peak around orbit 532, while the single node readout in orbit 1408 increases the RGS2 system peak.

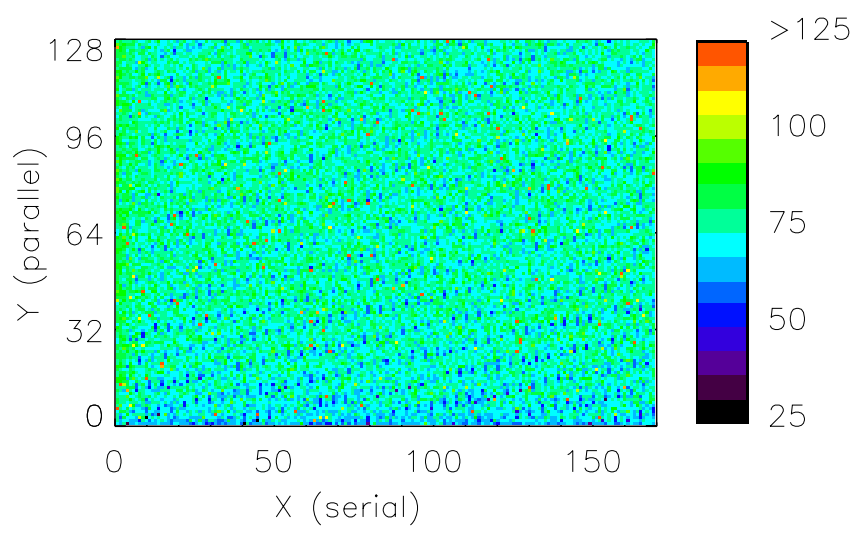

Fig. 14. Fixed pattern noise (RGS2:CCD1_C, two-node readout).

due to lowering the operational temperature (orbit 532 and 537), and the increase in dark current following the change to a single node read-out which resulted in longer integration times, in RGS2 (orbit 1408), are visible. From the change in noise floor between the two node read-out and the single node read-out corresponding to a change of about a factor 2 in read-out time, the dark current could be determined as $0.14 \mathrm{e}^{-} / \mathrm{bin} / \mathrm{s}$ where a bin is $0.00656 \mathrm{~mm}^{2}$.

In addition, we observe a small ( $\triangle \mathrm{ADC} \leq 30 \mathrm{ADU})$ systematic pattern in the maps of the noise floors in the CCDs (Fig. 14). Following the change to the single node read-out this pattern disappeared in RGS2, clearly indicating that this pattern is caused by cross talk between the two read-out chains. Although the effect is small compared to the energy resolution of the detectors ( $\geq 100$ ADU), and affects only $\approx 1 \%$ of all pixels it can, in principle, be corrected if the pattern is known. Unfortunately this noise pattern is not fixed and drifts slowly over time and is not correlated with any of the instrument operational steps (e.g. there is no synchronization with the start of the read-out of a CCD).

Following orbit 786 RGS1 CCD1 developed two hot spots (Fig. 15). The origin of these spots is not clear as it shows up symmetrically in one CCD only. If it is radiation damage or a change in stress in the Si due to aging of the gluing to the detector bench, one does not expect it to be symmetric for the two read-out nodes. It is possibly related to flat-band voltage shifts 

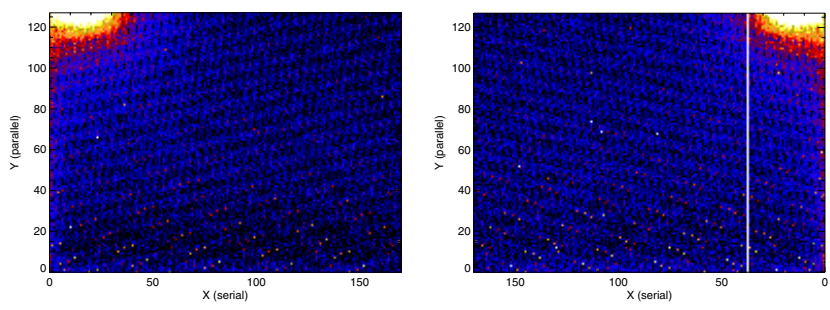

Fig. 15. Hot spots on RGS1 CCD1 with separate images of nodes C and D. The hot spot intensity is about 180 ADC units above the system level floor of about 100 ADC units.

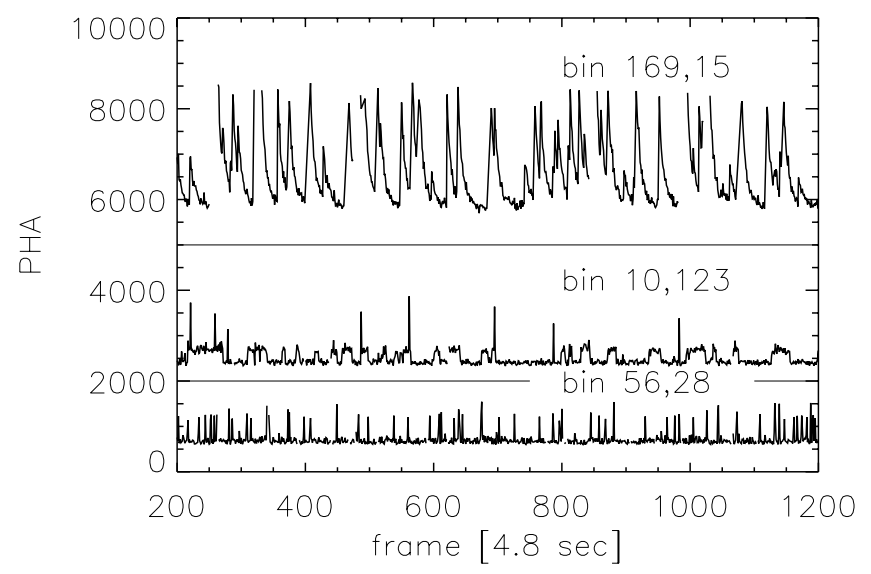

Fig. 16. Variations in temporal behavior of cosmetic defects (orbit 0440 , before the cooling). The top two panels are offset by 2000 and 5000 for clarity. The small gaps in the time sequence are due to the onboard processing where patterns which are not consistent with the expectation for X-rays will be rejected to reduce the telemetry load.

of the CCD due to radiation damage but this can not be verified. Fortunately this feature is limited to this single CCD only and its area does not increase over time. The onboard software excludes these areas from processing.

\subsection{Cosmetic defects}

Local defects and damage by cosmic rays in the depleted silicon material can give rise to hot pixels and columns. Although the CCDs were selected based on the absence of hot pixels, the instrument includes some CCDs with hot pixels and hot columns (see Table 7). The presence of these cosmetic blemishes is expected to vary over the life time of the mission. Due to radiation damage new hot pixels or columns can appear, and also flickering pixels (also called random telegraph noise) can be expected. Due to the mobility of the originating charge traps some hot pixels may also disappear over the mission lifetime. This is illustrated in Fig. 16 where the charges of a few noisy bins are shown. Bins with a content below the lower threshold (e.g. only read-out noise and dark current) are rejected onboard and have a typical pulse height of 100-120. The variation in the patterns is huge, varying from fast damping to a somewhat bi-polar state. Following the cool down (see later) the effect of these defects in the crystal was significantly reduced.

Searching for these cosmetic blemishes has been fully automated and we adopted an approach to reject all potential cosmetic blemishes. The signature of bad pixels and bad columns differs from the signature of astrophysical lines which cover more pixels. Therefore we compare the frequency that a given pixel or column is above the read-out noise with its neighbors,
Table 7. Hot columns and pixels $(3 \times 3$ OCB $)$ defined in the calibration files.

\begin{tabular}{|c|c|c|c|}
\hline Orbit & $\mathrm{CCD}$ & Column & Segment/comment \\
\hline \multicolumn{4}{|l|}{ RGS1 } \\
\hline \multirow[t]{9}{*}{1} & CCD1_D & 38 & hot column \\
\hline & $\mathrm{CCD} 2-\mathrm{C}$ & 166 & hot column \\
\hline & CCD3_D & 93 & hot column \\
\hline & CCD5_D & 119 & hot column \\
\hline & CCD7_D & 77 & hot segment $1-33$ \\
\hline & CCD7_D & 74 & hot segment $1-33$ \\
\hline & CCD9_D & 43 & hot column \\
\hline & & & suspect: \\
\hline & & & $\begin{array}{l}27 \text { pixels, } 5 \text { segments, } \\
5 \text { columns }\end{array}$ \\
\hline \multirow[t]{3}{*}{276} & CCD1_D & 38 & hot column \\
\hline & CCD3_D & 93 & hot column \\
\hline & $\begin{array}{l}\text { CCD9_D } \\
\text { all }\end{array}$ & 43 & $\begin{array}{l}\text { hot column } \\
\text { suspect: same }\end{array}$ \\
\hline \multirow[t]{5}{*}{536} & CCD1_D & 38 & hot column \\
\hline & $\mathrm{CCD} 3 \mathrm{D}$ & 93 & hot column \\
\hline & CCD9_D & 43 & hot column \\
\hline & & & suspect: \\
\hline & & & 5 pixels after cooling \\
\hline \multirow[t]{2}{*}{543} & CCD1_D & 38 & hot column \\
\hline & all & & suspect: 5 pixels \\
\hline \multirow[t]{4}{*}{1110} & CCD1_D & 38 & hot column \\
\hline & & & suspect: 4 columns \\
\hline & CCD1_C & $1-31$ & suspect segment $121-128$ \\
\hline & CCD1_D & $1-31$ & suspect segment $121-128$ \\
\hline \multirow[t]{4}{*}{1417} & CCD1_D & 38 & hot column \\
\hline & CCD1_C & $1-31$ & hot segment $121-128$ \\
\hline & CCD1_D & $1-31$ & hot segment $121-128$ \\
\hline & all - & & suspect: 4 columns \\
\hline \multicolumn{4}{|l|}{ RGS2 } \\
\hline \multirow[t]{3}{*}{1} & CCD9_C & 94 & hot column \\
\hline & CCD9_C & 147 & hot segment \\
\hline & & & $\begin{array}{l}\text { suspect: } 1 \text { column } \\
\text { and } 20 \text { pixels }\end{array}$ \\
\hline \multirow[t]{3}{*}{532} & CCD9_C & 94 & hot column \\
\hline & all & & suspect: \\
\hline & & & 1 pixel and 1 segment \\
\hline 1110 & CCD9_C & 94 & hot column \\
\hline & & & suspect: 7 columns \\
\hline
\end{tabular}

Notes. Suspect pixels/segments are pixels/segments which are rejected on the ground but are not persistent over the mission lifetime. The orbit is the start of the validity interval of the corresponding "BadPix" calibration file (see Table A.1).

neglecting the actual pulse height value of a given bin. If this frequency exceeds its expectations based on a binomial error propagation, the bin is rejected $\left(N>M+\sigma_{p} \sqrt{N\left(1-\frac{N}{F}\right)+M\left(1-\frac{M}{F}\right)}\right.$ with $N$ the total number of counts in a given pixel, $M$ the minimum of the total counts in the neighbouring pixels, $F$ the total number of frames and $\sigma_{p}$ is set at 5. For the rejection of hot columns we use a similar approach.

The approach to reject cosmetic blemishes is rather conservative, but as long as it is properly taken into account in the computed exposure for each observation, it ensures that hot pixels/columns do not cause any artificial emission lines. This method translates into an exposure map with large pixel to pixel variations affecting the effective area curves (Fig. 34). In Fig. 17 we display the "dead" area of the two detectors over the lifetime of the mission. The step-wise increase in the early part of the 


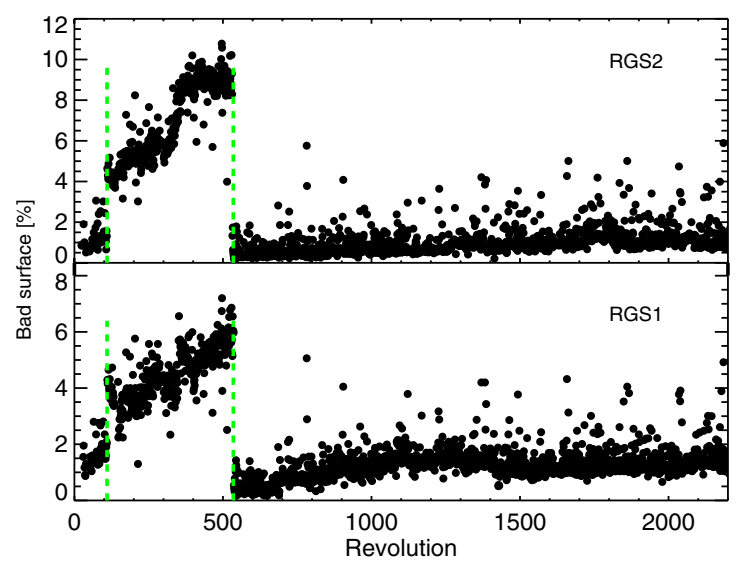

Fig. 17. Bad area per detector as detected by the standard monitoring tools. The first increase corresponds to a major solar flare in orbit 110 , the drop around orbit 535 is due to the cooling of the detectors from $-80^{\circ} \mathrm{C}$ to $-110^{\circ} \mathrm{C}$. Individual outliers are due to peculiar observational circumstances, like e.g. periods of very high background, observations of very bright sources, or very short observations.

mission corresponds to solar activity. Following the cool down of the detector the number of hot items reduced by a factor 5-10 and we expect that the total affected area will stay below $4 \%$ over the next 10-15 years, depending of course on the actual solar activity in the future.

\subsection{Warm/cool pixels}

As remarked before (Sect. 5.4), defects in the depleted region may cause local higher dark current, or extra charge traps, leading to hot and cool pixels respectively. Hot pixels are discarded, but many pixels will only show a slightly elevated dark current, which is added to the charge of recorded X-ray events, potentially shifting them outside of the pulse height selection area. Cool pixels will lower the recorded charge of events. To mitigate this effect, averages are made of CCD full frame images, recorded during three consecutive orbits around the orbit of the observation being processed. These averages are used to subtract the system peak level on a pixel by pixel bases, which will correct for the different system level peaks of hot and cool pixels.

\subsection{Charge transfer inefficiency}

Ionizing particles from cosmic rays can also cause traps in the Si lattice structure at the layer where the charge is shifted from pixel to pixel to the output node. These traps are characterized by the trapped charge relative to the total charge packet, the probability that the charge remains trapped during the charge transfer, and the probability that the charge will be emitted during the time between charge packets. Although this effect depends on the time since the last charge transfer took place through the pixel, in first approximation we can describe this by an average charge transfer inefficiency (CTI) that neglects the pixel to pixel variations and pixel history. The unit of CTI is defined as the fractional loss of charge per physical charge transfer on the CCD. On the RGS CCDs there are up to 1024 transfers in the CCD X-direction, in single node readout, for the most distant pixel to reach the readout node. Our general CTI model is justified because the CCD pulse height distribution is only used to separate the orders and not for the spectral response of the instrument; our CTI correction does not need very high accuracy. The uncertainty in the total CTI correction for a pixel corresponds to $1 \%$ in the total charge for an average CTI per transfer of $10^{-5}$, to keep it the same order of magnitude as the gain error. A wider selection of the CCD response function results in a smaller error, but leads to more background. In addition to traps, the CTI is also affected by the voltages applied to the clocks when shifting charge through the CCD. Since we know the energy of the incident photons from the position on the detector, we can tune the voltages for the clocks and/or calculate the CTI corrections.

After failure of the RGS1 CCD7 readout chain CCD clock voltages were reduced to limit stresses on the components (orbit 168, Table 12). These clock voltage reductions were further tuned for RGS2 CCD2 which was done in orbits 192 and 537 , and the clock bias of RGS1 CCD2 needed adjustment in orbit 1389 .

The charge transfer inefficiency is calculated independently for the parallel transfers (transfer of the image section to the storage section or of a row in the storage section to the read-out row) and for the serial transfers (transfer through the read-out row toward the read-out amplifier).

For the parallel transfers the CTI is deduced from the measurement of a strong point source (Mrk 421) at different positions in the cross dispersion direction. The difference in collected charge for different positions in the cross dispersion direction is only due to the CTI loss, as the deposited energy is given by the grating dispersion equation. This can be simply converted into a CTI calibration model.

For the serial transfers a slightly different approach is followed. CTI during readout causes the collected charge to drop depending on the number of charge shifts, given by the CCD column. Using the known energy of the photons from the dispersion relation of the grating, the relative change in charge deposited by the incoming photons over the CCD is known. The additional charge loss per transfer gives the CTI model.

The parallel and serial CTI are small (few times $10^{-5}$ ). Prelaunch values were around $0.9 \times 10^{-5}$. Every two years special calibration measurements are executed to calibrate the CTI. Additional calibrations were performed immediately after cooling of the RGSs and following the change from double to single node readout of RGS2. Table 8 shows a moderate increase of the CTI of $10^{-5}$ over 1000 orbits but the CCD to CCD variations are larger $\left(\mathrm{a}\right.$ few $\times 10^{-5}$ ). These parameters can be determined to about $0.5 \times 10^{-5}$ which corresponds to an uncertainty of the energy loss of $\lesssim 0.5 \%$.

Near the edges of the CCDs the parallel CTI is significantly larger. To reduce the dead space between adjacent CCDs in the detector array, the CCDs were sawn at the two sides, causing additional stress and hence defects in the Si (Fig. 18). During the early part of the mission the parallel CTI near the CCD edges is $\sim 20 \times 10^{-5}$, and it reduces to a few times $10^{-5}$ after the cooling of the camera in orbit 539. Over time the CTI increases again.

The change in CTI was independently verified by comparing the position of the onboard calibration line positions as function of orbit. This is shown for the Al-K $\alpha$ line in Fig. 19 where the position of the Al-K onboard calibration source is shown before any gain and CTI correction. Apart from the jumps associated to CCD tuning, cooling and change to single node readout, a gradual decrease in pulse height of $3 \%$ over the lifetime of the mission is observed. This corresponds to a change in CTI of $10 \times$ $10^{-5}$ over 1600 orbits, consistent with the change of $5 \times 10^{-5}$ in 800 orbits for the higher CCD channels, as shown in Fig. 18.

\subsection{Optical sensitivity}

The mirrors and gratings are near perfect reflectors for UV/optical light and this results in additional charge on the 
Table 8. Average CTI degradation per camera as retrieved from the calibration files (CCF) and variation (maximum difference) over all CCDs, in between brackets.

\begin{tabular}{lrrrl}
\hline \hline RGS & Orbit & Serial CTI $\left(10^{-5}\right)$ & Parallel CTI $\left(10^{-5}\right)$ & Comment \\
\hline RGS1 & 30 & $3.45(2.90)$ & $0.94(0.05)$ & pre launch data \\
& 260 & $4.16(2.61)$ & $4.26(1.60)$ & first measurement in orbit \\
& 536 & $5.05(4.30)$ & $4.07(2.00)$ & post cooling \\
& 807 & $5.07(6.53)$ & $6.55(4.10)$ & \\
& 1397 & $4.89(3.83)$ & $7.68(4.41)$ & \\
& 1839 & $4.96(3.53)$ & $8.92(4.98)$ & \\
& 2021 & $5.92(5.42)$ & $11.13(3.70)$ & \\
\hline RGS2 & 25 & $3.29(3.14)$ & $0.95(0.05)$ & pre launch data \\
& 260 & $3.55(5.51)$ & $3.05(2.98)$ & first measurement in orbit \\
& 532 & $3.87(2.96)$ & $2.14(2.81)$ & cooling to - 115 ${ }^{\circ} \mathrm{C}$ \\
& 536 & $3.48(2.82)$ & $2.42(2.38)$ & cooling nominal \\
& 807 & $3.17(2.65)$ & $6.11(3.73)$ & \\
& 1405 & $2.86(1.17)$ & $8.06(3.71)$ & to single node readout \\
& 1839 & $2.71(1.04)$ & $9.67(4.96)$ & \\
2021 & $2.58(1.82)$ & $11.09(5.29)$ & \\
\hline
\end{tabular}

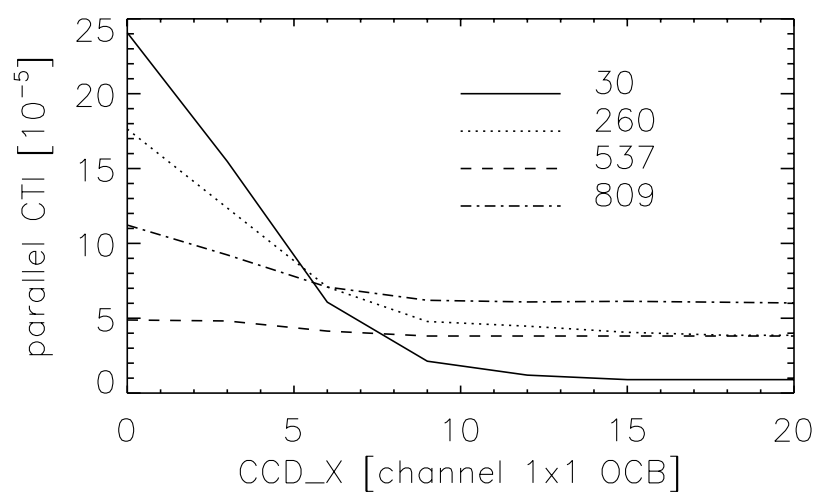

Fig. 18. Average parallel CTI for RGS1 near the edges for different orbits. The uncertainty towards the edge increases towards about $5 \times 10^{-5}$. For channels $>10$ the uncertainty is of the order of $1 \times 10^{-5}$.

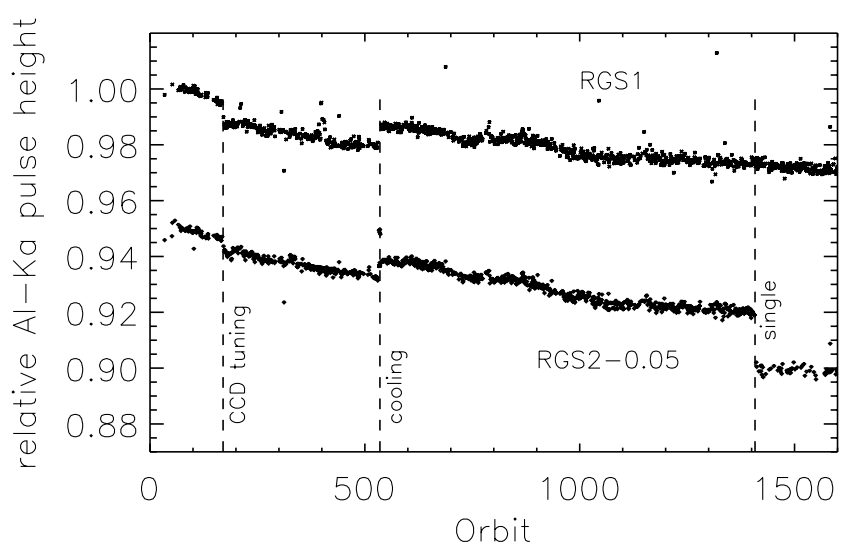

Fig. 19. Change in charge transfer efficiency as reflected by the gradual decrease of the uncorrected pulse height value for events from the onboard Al-K $\alpha$ calibration sources. Major events: tuning of the CCD settings, cooling of the detector and switch to the single node read-out for RGS2, cause sudden shifts in CTI. Occasional outliers are caused by peculiar observations like e.g. bright sources, observations with high background and very short observations.

detectors for optically bright stars. The energy assigned to the $\mathrm{X}$-ray photons increases by a corresponding amount and therefore is incorrectly interpreted. On the EPIC cameras this is known as "optical loading". The filter wheel in front of the EPIC instruments allows the user to reduce the optical light.

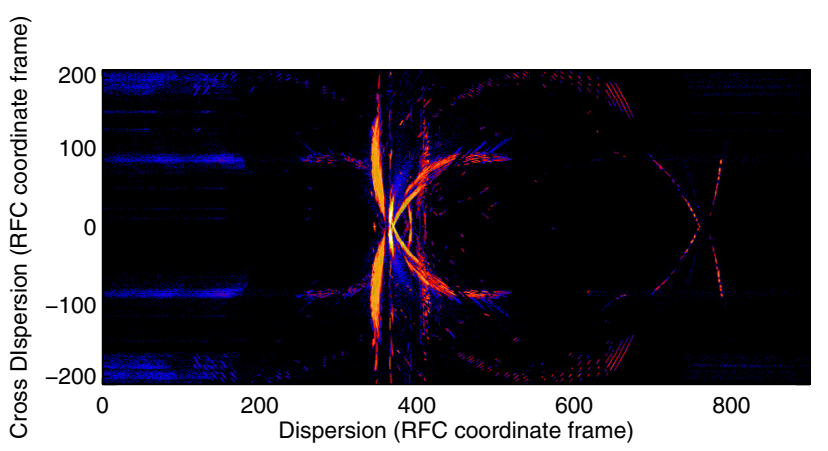

Fig. 20. Simulated image for a source at -1.5 degree off-axis in the direction of increasing incident angle on the grating. The projected photons near $Z=400$ (the narrow lines) are double reflections from the mirror. The fan like shape is due to single or double reflections from the mirror and a single reflections from the grating. In the RFC coordinate frame position $(0,0)$ is de center of CCD5 which is the position of the grating first order blaze wavelength. The coordinates are in units of $0.01 \mathrm{~mm}$.

For RGS, with its elongated focal plane, a different solution is used. For sources within the field of view the specular reflected photons are imaged on the zeroth order position of the grating, $40 \mathrm{~mm}$ away from the CCD bench, and they do not interfere with the dispersed X-ray spectrum. Because optical radiation has much longer wavelengths, the higher orders of optical light will end up far beyond the CCD bench. However, light from bright off-axis sources can interfere with the recorded spectrum (Fig. 20).

Based on simulations we calculate the corresponding effective area for different off-axis angles and position on the detector. This is shown in Fig. 21. Based on this sensitivity we verified whether possible contributions from the sky would affect the RGS response. As expected contributions from bright stars dominate the sensitivity, relative to the night glow features and diffuse night sky. Very bright stars near the field of view can be avoided by proper scheduling, as the optical sensitivity depends only on the incident angle with respect to the gratings. Without further scheduling constraints to $m_{v} \leq 5,4 \%$ of the observations would be optically contaminated. Also the population of weaker field stars adds to the optical contamination. Reduction of the optical load is required to keep the contribution of this specular reflected star light to less than 1 electron per readout. This reduction varies from $10^{-4}$ near the optical axis to $10^{-2}$ 


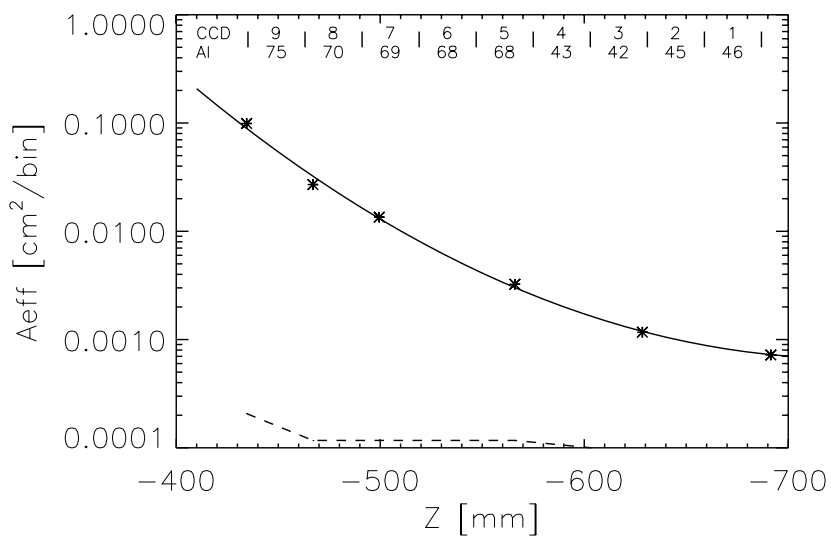

Fig. 21. Global stray light sensitivity of RGS as modified by the varying Al filter thicknesses (in nm). Separate CCDs and their Al filter thicknesses are indicated (top). The sensitivity for focused (solid line) and non focused light (dashed line) is given separately. The asterisks indicate ray-trace model calculations for the indicated positions on the CCDs.

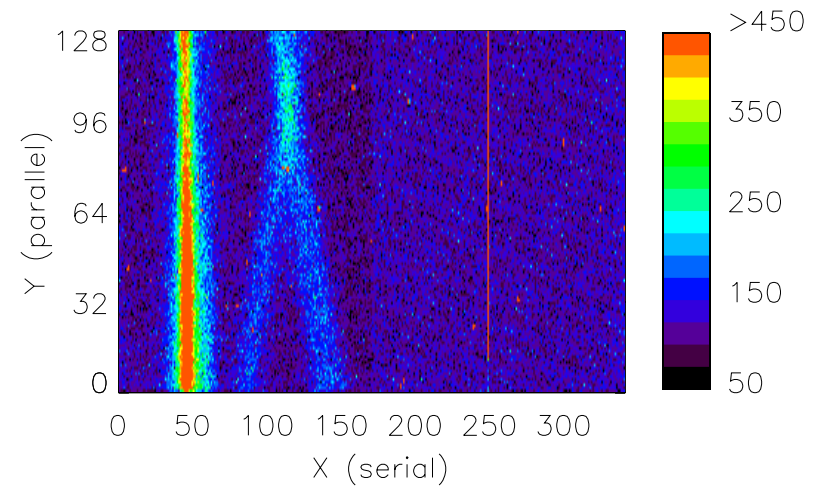

Fig. 22. Recorded intensity distribution on CCD 3 with Canopus at -2 degree off axis.

for the CCDs at the largest distance to the optical axis and has been achieved by an Al layer deposited directly on the CCDs. To compensate for the difference in sensitivity as function of the dispersion direction, we used a thicknesses of $75 \mathrm{~nm}$ for CCD9 and CCD8, $68 \mathrm{~nm}$ for CCD7, CCD6 and CCD5, and $45 \mathrm{~nm}$ for the other CCDs.

The suppression of UV/optical light was verified in orbit by observing Canopus an F0 star with $m_{v}=-0.72$ between -3 and +2 degree off axis along the dispersion direction. The observed image (Fig. 22) has the correct shape and its intensity is within a factor 2 from the model. Considering the approximations used (a $1 \mathrm{~nm}$ change in $\mathrm{Al}$ filter thickness may change the transmission up to $15 \%$ ) this is reasonable and the observation strategy of an avoidance angle for bright sources is sufficient to neglect the effect of optical/UV light on the detector response.

\subsection{Stability detector response}

After applying all correction factors for the evolution of the detector response (CTI, gain, hot columns, read-out noise and dark current and the evolution of hot pixels and columns) we expect that the calibrated CCD response is accurate over the lifetime of the mission. For a detector behind a dispersive element this can be easily verified as the incident energy of each photon is known. In Fig. 23 we show the calibrated CCD redistribution function for one energy at the start of the mission and after 10 years in operations.

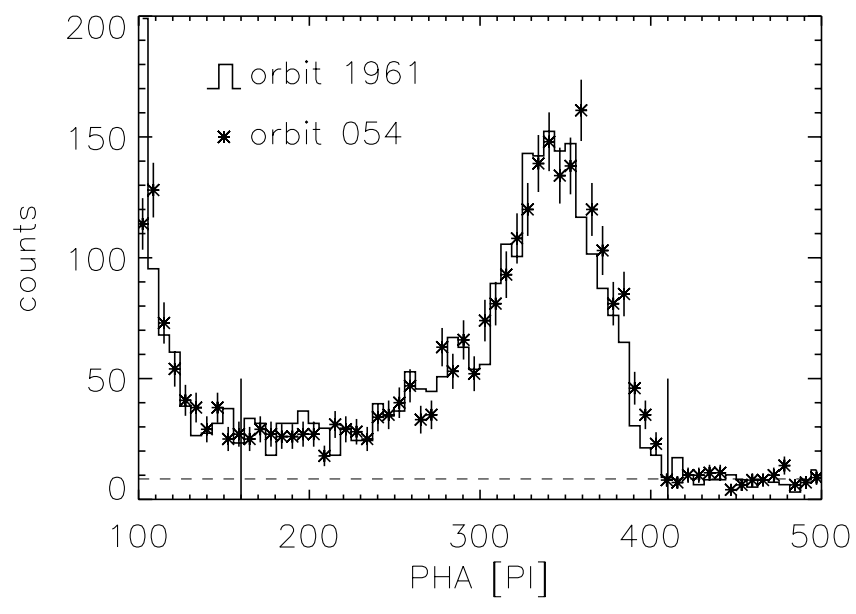

Fig. 23. In-orbit CCD response at $\beta=0.0715$ radians (C VI line) for Capella observations in orbit 54 and 1961. The errors are only shown for orbit 54.

The stability of the detector response, after applying the appropriate corrections is very good and the effect on the effective area is negligible $(<0.25 \%)$ assuming that the proper pulse height selection windows $\geq 95 \%$ are applied.

\section{Gratings}

\subsection{Grating model}

The grating response describes the intensity distribution of monochromatic radiation as function of outgoing angle $\beta$ and its component perpendicular to it. The two major contributions are the flatness and the scattering properties of the gratings. Also the grating to grating alignment is a major component.

From scalar diffraction theory one can calculate the total integrated scatter (TIS) out of the line core (Kahn et al. 1996). This is given approximately by:

$T I S=k^{2} \sigma^{2}(\sin \alpha+\sin \beta)^{2}$

where $k=2 \pi / \lambda$ is the wave number of the radiation and $\sigma^{2}$ is the mean square deviation of the surface which follows from a simple analytical function that is characterized by $\sigma$ and a scale length $l$. This expression (13) is valid in the small amplitude limit where $\lambda \gg \sigma \sin (\alpha)$ and where the deflection angles of the surface are small compared to the critical graze angle for reflection. We get a good description of the grating response using two components: an incoherent large scale component with a correlation length of $1 \mu \mathrm{m}$ and an rms of 8 to $14 \AA$ depending on the grating master and a coherent short scale component with a correlation length of $0.29 \mu \mathrm{m}$ and an rms of $15 \AA$. This second component is due to a sinusoidal wave on top of the groove structure and has been verified by scanning tunneling micrograph images.

For the diffraction efficiency we use an exact approach which solves the vector electromagnetic equations in the space above the grating subject to the periodic continuity relations imposed by the groove surface. Based on synchrotron measurements we verified the blaze angle, which is the angle where the efficiency peaks.

\subsection{Gratings calibrations}

Calibrations of the gratings included two steps: (a) verification of the grating response model at a synchrotron and (b) measurements of the efficiency of each grating separately at a few wavelengths. 


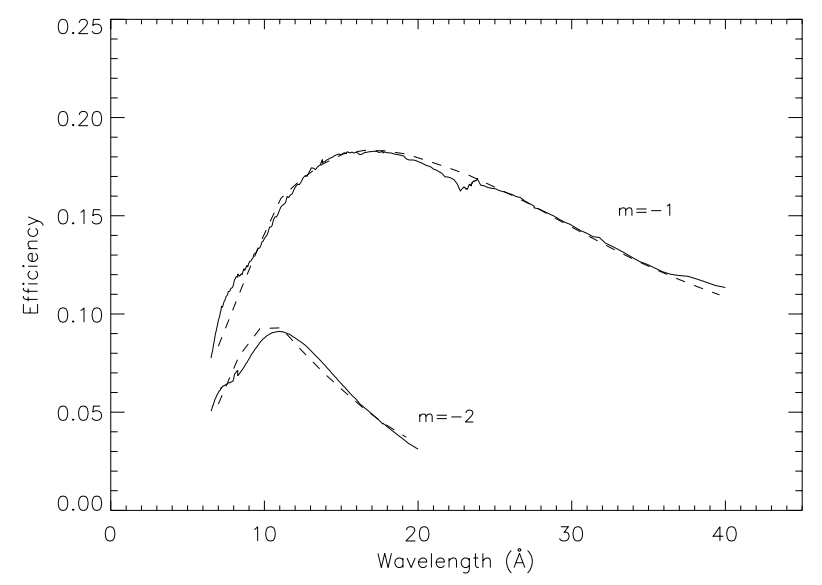

Fig. 24. Efficiencies for first and second order as measured (solid curve) and as modeled (dashed curve), for a single grating (den Herder et al. 2001).

The single grating response was verified by measuring the efficiency of a single grating at the Bessy synchrotron facility in Berlin (Fig. 24). Clearly there is good agreement, allowing the blaze angle and the scattering contributions as free parameters. Near the oxygen and carbon edges a thin layer of hydrocarbons on the surface of the grating is not included in our model, causing small differences. At short wavelengths the model, based on a scalar approximation of the diffraction theory (Cottam et al. 1998) breaks down.

All gratings were calibrated as part of the screening of replicated gratings: their efficiency was measured at 8.34 (Al-K $\alpha$ ), $13.34(\mathrm{Cu}-\mathrm{L} \alpha), 17.59(\mathrm{Fe}-\mathrm{L} \alpha)$ and $23.62 \AA(\mathrm{O}-\mathrm{K} \alpha)$ and scattering measurements were performed at the Al-K line. For a few gratings additional measurements of the inter-order scattering were carried out. The selection of flight gratings was based on a grading scheme where poorer candidates were rejected on the basis of their $m=-1$ efficiencies and the replica flatness (for RGS1: $\epsilon_{\mathrm{Al}}>0.09$ or $\epsilon_{\mathrm{Cu}}>0.15$ and flatness $<15 \mu \mathrm{rad}$ and for RGS2: $\epsilon_{\mathrm{Al}}>0.085$ or $\epsilon_{\mathrm{Cu}}>0.15$ and flatness $<9 \mu \mathrm{rad}$ ).

The integrated reflection grating assembly was tested at the Panter facility at MPE. This test included two components: checking the grating assembly by partial illumination of the RGA and measuring the LSF (mirror + gratings) at some energies.

The partial illumination was achieved by blocking the mirror entrance aperture except for a small section, using the so-called Glücksrad. Following these tests we concluded that apparently the reference point for this RGA was not set properly during assembly. This meant that for the first RGA we had to modify the orientation of the RGA with respect to the mirrors by a change in angle $(\Delta \alpha=+4.56$ arcmin and $\Delta x=7 \mathrm{~mm})$. In addition a rotation in the cross dispersion direction, around the $Z$-axis, was applied to reduce the scattering from the supporting ribs on the gratings $(\Delta \theta=+1.71$ arcmin). For the second unit such modification was not required. Following these modifications the alignment of the gratings was tested at the Panter long beam facility at MPE and these were consistent with the expected results.

The full verification of the LSF is described in Sect. 7 where we show the results for the calibrations of the mirror and grating combination.

\subsection{In-orbit grating response verification}

The grating response in the dispersion direction is not easily verified in flight as it includes a convolution with the incident

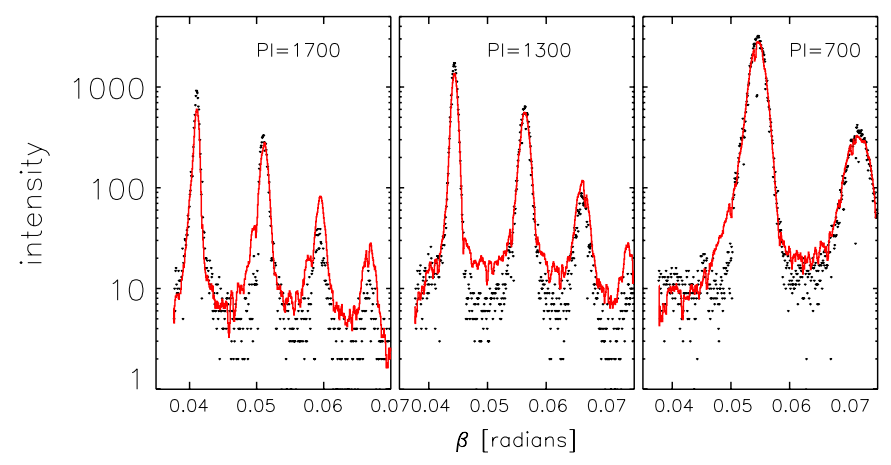

Fig. 25. Modeled and measured $\beta$ distributions for given energies expressed in CCD pulse height $(\mathrm{PI})$, where $1 \mathrm{ADU} \approx 1 \mathrm{eV}$. This corresponds to a horizontal slice through the PHA-Beta plot in Fig. 2. The peaks correspond to the different orders, starting from order -1 on the left of each plot.

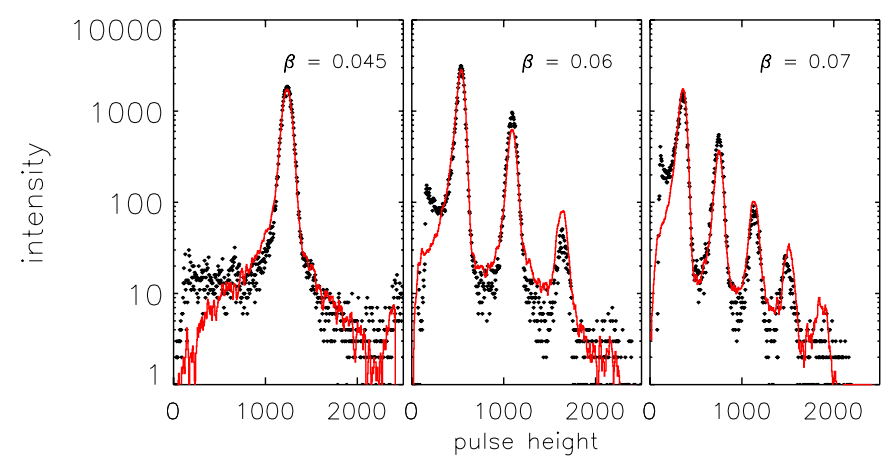

Fig. 26. Modeled and measured CCD pulse height distributions expressed in $\mathrm{CCD}$ pulse height $(\mathrm{PI})$, where $1 \mathrm{ADU} \approx 1 \mathrm{eV}$, for given $\beta$ angles in radians. This corresponds to vertical slices in the PHA-Beta plot in Fig. 2. The peaks correspond to the different orders, starting from order -1 on the left of each plot.

spectral shape of the observed object. When the spectral continuum has a strong gradient, or strong lines are present, spectral intensity will spill to neighboring energy bands and mix with the grating response when the spectrum is integrated in cross dispersion direction along the dispersion axis. A major factor in the grating response is the scattering by the grating due to irregularities and surface roughness of the grating (see Fig. 28).

The grating response was first verified using Mrk 421 for a number of selections in the dispersion angle $\beta$ and in pulse height (Figs. 26 and 25). The spectrum as a function of dispersion angle $(\beta)$ clearly shows the different orders and the scattering from the gratings in between the orders. There is good agreement between the data and the modeled response for the first and second order after applying the scattering component.

This scattering also occurs in the cross-dispersion direction. In the two-dimensional data slice of cross-dispersion versus the dispersion, or spectral axes, the cross dispersion profile is affected by the scattering of neighboring spectral bin intensities. However, its fractional amplitude is suppressed by a factor of 20 compared to the scattering amplitude along the dispersion direction due to the difference in relative incidence angle between the dispersion and cross-dispersion direction. When the spectrum is collapsed on the cross dispersion axis the crossdispersion profile is largely a convolution of the mirror PSF with the grating response. Figure 27 shows the verification of this scattering component. Overall, good agreement is observed, confirming the validity of our scattering model. 

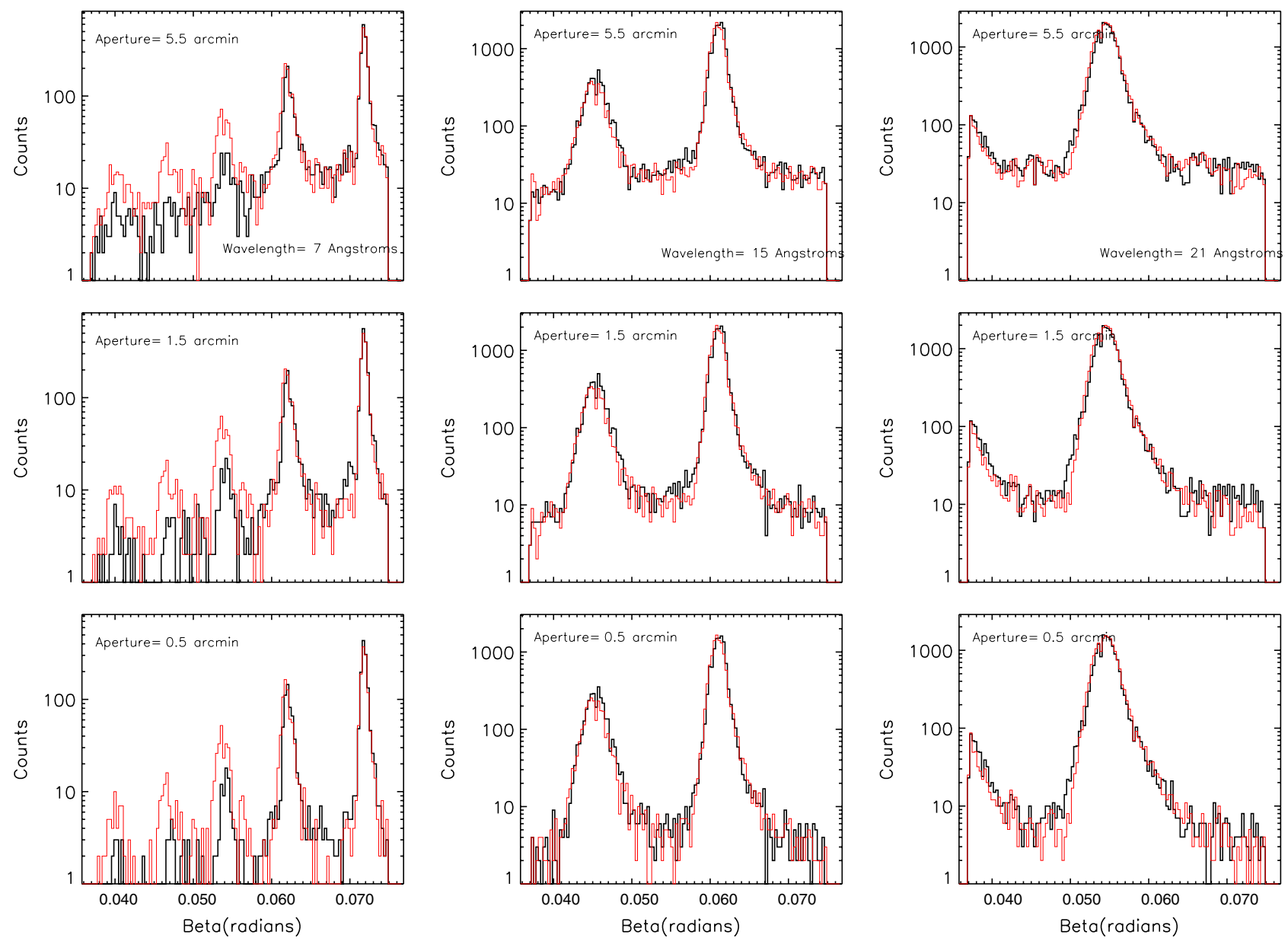

Fig. 27. Comparison of modeled response (red line) with observations (black line) for different cross dispersion apertures (ranging from 0.5 to 5.5 arcmin, bottom to top), shown as intensities versus dispersion angle (Beta) for different CCD pulse height selections (columns left to right) at 7,15 and $21 \AA$, or $1.7,0.8$ and $0.6 \mathrm{keV}$.

\subsection{Scattering of low energy protons}

The low energy protons encountered by XMM-Newton during its orbit form a potential risk for the performance of the CCD detectors (see Sect. 5.2). These protons are scattered and focused by the mirrors onto the XMM-Newton instruments (Aschenbach 2007). For the RGS an extra reflection by the gratings is required in order for the protons to hit the CCDs. To estimate the efficiency of this process, a sample grating was put in a soft proton beam at the Harvard university, Cambridge accelerator for materials science (Rasmussen et al. 1999). It was found that for protons of $1.3 \mathrm{MeV}$, the CCDs would acquire per $\mathrm{cm}^{2}$ only $10^{-5}$ part of the radiation dose on the mirror entrance due to scattering off the grating.

It was concluded that for the RGS the required extra reflection by the gratings, in combination with the lower vulnerability of our back illuminated devices for these soft protons, offers sufficient protection. In principle the instrument can even be left in operational mode during the periods of high radiation caused by soft protons.

\section{Linespread function}

The LSF is determined by folding of the mirror response with the grating response, taking into account the effect of data selections in the detector. This is schematically illustrated in Fig. 28 where the different components are shown. The sharp symmetric Lorentzian profile describes the mirror response. After folding with the grating response the distribution shows a narrow angle scattering component (Gaussian shoulders in the distribution) and additional large angle scattering wings. The downward spikes in this spectrum are due to discarded hot columns. The small step function is due to the limited range over which certain scattering components are computed. Beyond this range this component becomes insignificant, and only the total wide angle scattering flux is needed to compute the total amount of flux lost from the center of the distribution, to get the proper effective area. Applying the data selection of the CCD the shape of this distribution becomes asymmetric in the wings.

The LSF was verified in various stellar sources (Capella, Procyon, HR1099, AT Mic and $\lambda$ And). The instrument behaves slightly better than predicted by the folded responses, in the sense that the line peak is slightly narrower. This is modeled by a small $(\approx 1 \mathrm{~m} \AA$ equivalent for 1 st order $)$ manual modification of the figure parameters of the grating description (see LINESPREADFUNC ccf calibration file).

A typical example of the LSF response is shown in Fig. 29. The estimated contribution due to the background and the stellar continuum causes the largest uncertainty in the modeled wings of the line.

Using relatively clean emission lines ( Ne X Ly $\alpha$, O VIII Ly $\alpha$, Fe XVII, N VII Ly $\alpha$, and C VI Ly $\alpha$ ) we determined the resolving power $E / \Delta E$ for the two orders in the different instruments (Fig. 30). The resolving power is between 100 and 600 in the first order and is two times better in the second order. 


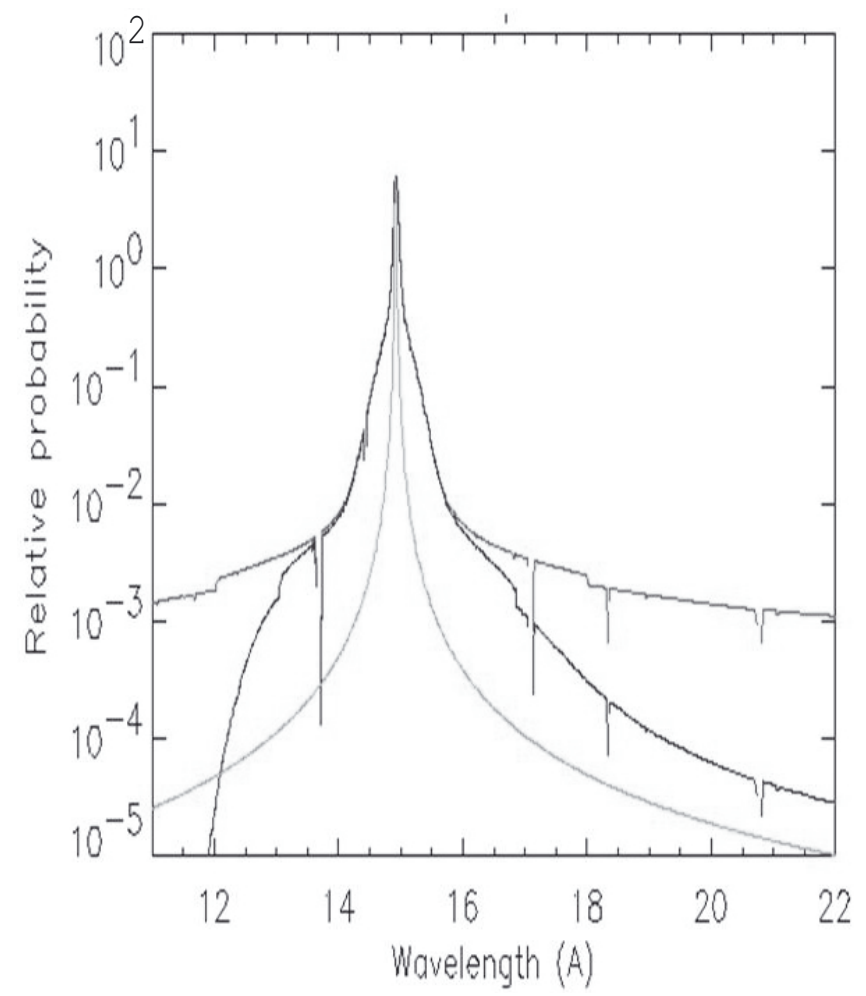

Fig. 28. Modeled linespread function showing the different contributions of the mirror (narrow peak), convolved with the small angle scattering (middle line) and wide angle scattering (top line).

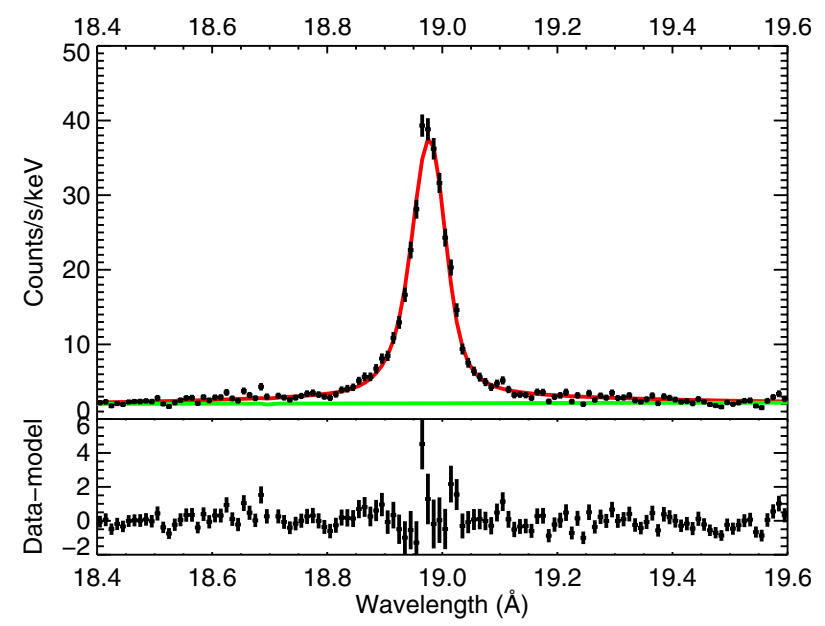

Fig. 29. Measured (points) and modeled linespread function (line) for the O VIII Ly $\alpha$ line in HR 1099 (18.97 $\AA$ ), for RGS2. The assumed stellar continuum background is indicated by the lower (green) line. The modeled line is shifted by $5 \mathrm{~m} \AA$, to fit the measured line position. This $5 \mathrm{~m} \AA$ shift is within the wavelength uncertainty and of the same order as the radial velocity shifts within the system which are between +40 and $-65 \mathrm{~km} \mathrm{~s}^{-1}$ (Bopp \& Fekel 1976), or about $\pm 3 \mathrm{~m} \AA$. The difference between the model and the data is shown in the bottom plot.

The modeled LSF and the observed LSF agree well after application of a correction factor for the internal alignment of the gratings of RGS1. Based on the spread of the measured resolution for a given wavelength (Fig. 30) astrophysical line broadening of more than $\Delta \lambda=6 \mathrm{~m} \AA$ or $10 \%$ of the line width of the instrumental profile can be detected significantly for strong lines $(S / N \gtrsim 25)$.

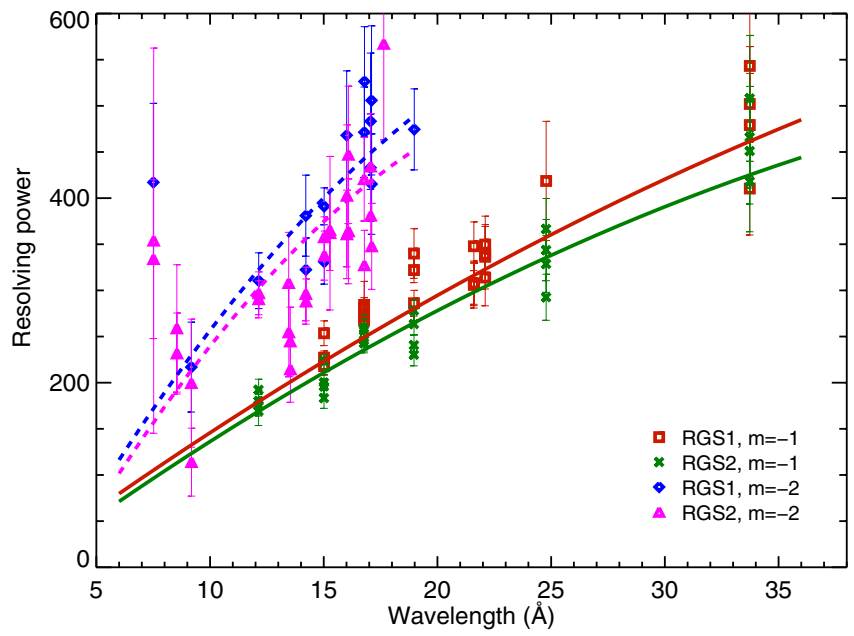

Fig. 30. Resolving power for RGS1 and RGS2 implemented in the calibration given by the response matrix (lines), and measured from individual line profiles (points), showing the spread around the model, and demonstrating that the line widths follow the trends defined by the physical model of the instrument.

Table 9. Lines used to calibrate the wavelength scale and LSF.

\begin{tabular}{llll}
\hline \hline Line & $\lambda_{\text {Laboratory }}$ & RGS1 LSF & RGS2 LSF \\
\hline Mg XII Ly $\alpha$ & 8.41 & no & no \\
Ne X Ly $\alpha$ & 12.134 & no & yes \\
Fe XVII & 15.015 & yes & yes \\
Fe XVII & 16.777 & yes & no \\
O VIII Ly $\alpha$ & 18.969 & yes & yes \\
O VII $w$ & 21.602 & yes & no \\
O VII & 22.101 & yes & no \\
N VII & 24.781 & no & yes \\
C VI Ly $\alpha$ & 33.736 & yes & yes \\
\hline
\end{tabular}

Notes. The last two columns indicate which lines have also been used for the separate RGS1 and RGS2 LSF calibrations.

\section{Wavelength calibration}

On the ground a full calibration of the wavelength scale with X-rays was not possible as the mirror-grating-detector combination has to be at different positions due to the finite source distance at the Panter facility at MPE. Therefore the wavelength calibration had to be performed in orbit.

\subsection{Wavelength scale}

Using a set of well defined emission lines the relative alignment of the three components, mirror, gratings and detector, has been determined. We have used relatively clean lines (Table 9) in a number of coronal sources during the performance verification phase: Capella (orbit 042, 046, 054), HR 1099 (orbit 025, 028, 031, 036) and AB Dor (orbit 091). In this analysis the barycenter corrections and corrections for the velocity of the spacecraft are included. Since SAS version 13 these corrections can be applied (but not by default) by the standard software analysis system.

The dominant factors in the wavelength scale are (a) actual pointing position relative to the source, (b) a rotation of the RGA around the $Y$-axis (changing the incident angle) and (c) a linear shift in the $Z$-direction (dispersion). This is an over constrained problem because the final shift in wavelength depends on a linear combination of $\sin \alpha$ and $\sin \beta$. We have chosen a solution 

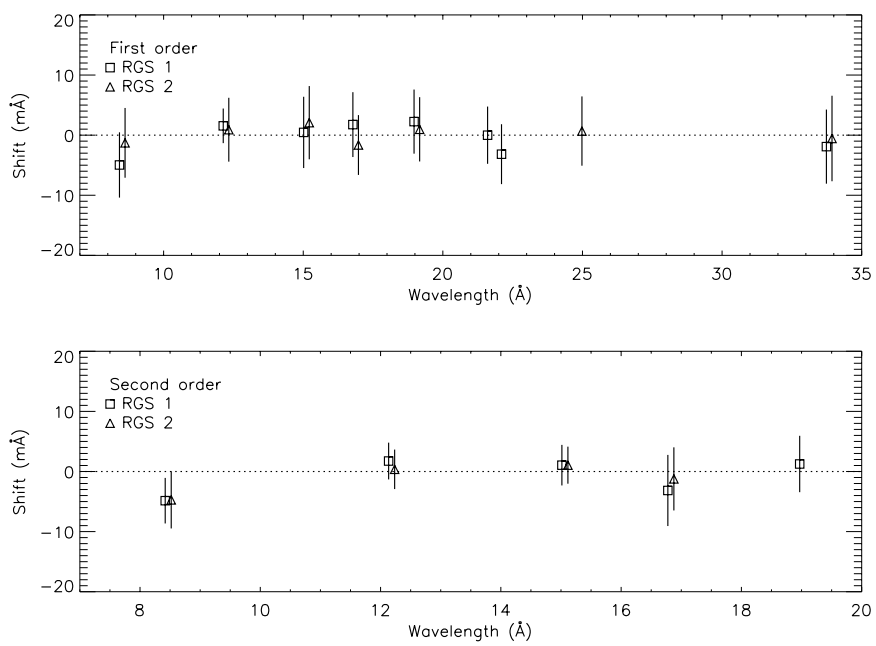

Fig. 31. Difference between expected and observed wavelengths as a function of expected wavelengths for RGS1 (squares) and RGS2 (triangles). RGS2 data have been slightly (horizontally) displaced for clarity. Error bars are the standard deviations of the statistics of the line fits. The spread of the data points seems somewhat smaller than the error bars indicate. There is a possibility this is due to correlations between spectral bins because of rebinning of the original CCD pixels, which might cause the statistical error to be somewhat overestimated.

where we fixed the incident angle on the gratings to the preflight calibrations, and modified the offset pointing (boresight) and the shift of the detector (810 and $490 \mu \mathrm{m}$ for RGS1 and RGS2 respectively).

It was noted that the variation between observed and theoretical line positions was larger than expected based on the built accuracies of the instrument. Bending of the telescope tube, the structure between the mirror platform and the detector, could have caused this variation. However, this was ruled out because the focus of the mirrors, as recorded by the EPIC instrument, is perfectly stable. After collecting a large set of observations this variation turned out to depend on the angular distance between the Sun and the spacecraft pointing direction, the "Sun angle" (SA). Considering that the mirrors are mounted at the intersection plane between the paraboloid and hyperboloid, a difference in temperature of less than 0.5 degree between observations with a different SA can explain a shift of up to $2 \mathrm{~m} \AA$. This is well within the required temperature stability of the satellite of 1 degree for the mirror and grating combination. This effect is included in the calibration since version 13 of the SAS. In Fig. 31 the accuracy of the wavelength scale is illustrated. Clearly there is no wavelength dependency after the calibration has been applied.

Recently it was discovered that there appears to be a seasonal pattern in the XMM-Newton pointing accuracy, with an amplitude of $1-1.5$ arcsec. This bore-sight variability, originally noted in the EPIC instruments, is thought to be caused by startracker instabilities. These offsets are taken into account since September 2012.

\subsection{Wavelength accuracy}

To verify the ultimate accuracy of the wavelength calibration we have used the same lines for a large number of observations of 4 coronal sources and calculated the distribution of the difference between the expected and measured positions for the first and second order lines (Fig. 32). Table 10 shows the accuracies and line shifts obtained for the lines used in the calibrations.
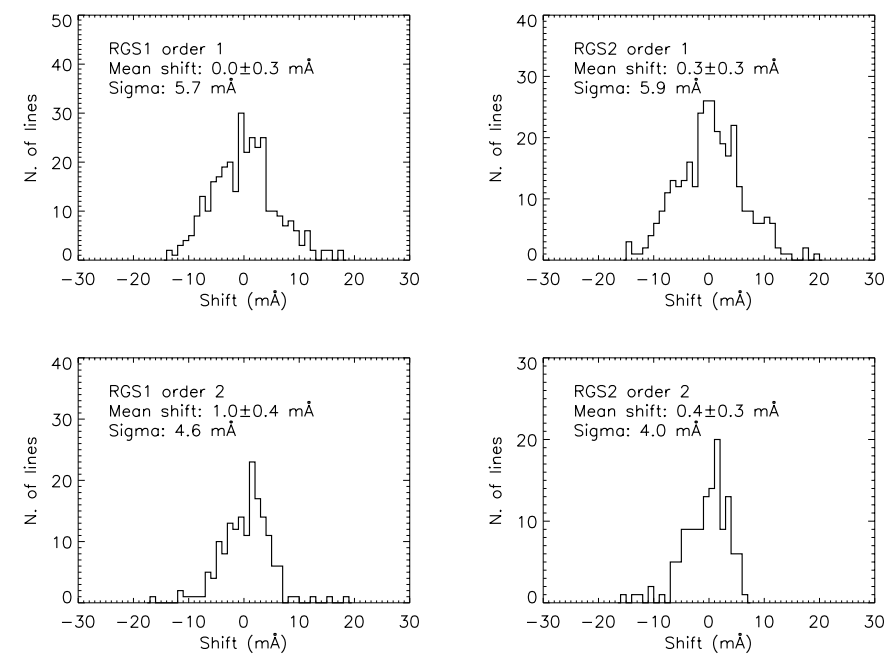

Fig. 32. Distribution of the difference between the measured and the theoretical wavelength for nine emission lines in 56 observations of four stars (Capella, AB Dor, HR 1099 and Procyon).

Table 10. Wavelength accuracy in $\mathrm{m} \AA$ for the applied corrections.

\begin{tabular}{lccc}
\hline \hline Spectrum & $a$ & $b$ & $c$ \\
\hline RGS1 order 1 & $4 \pm 8$ & $2 \pm 7$ & $0 \pm 6$ \\
RGS2 order 1 & $9 \pm 8$ & $7 \pm 7$ & $0 \pm 6$ \\
RGS1 order 2 & $2 \pm 5$ & $1 \pm 5$ & $1 \pm 5$ \\
RGS2 order 2 & $4 \pm 5$ & $3 \pm 4$ & $0 \pm 4$ \\
\hline
\end{tabular}

Notes. a) Fixed bore-sight, no further corrections; b) fixed boresight + barycenter and stellar velocity corrections; c) variable boresight + barycenter and stellar velocity corrections and Sun angle (SA) corrections.

Applying all known corrections, shown in the last column of Table 10, line shifts are zero with a spread for first order of $5 \mathrm{~m} \AA$. Thus for any observed line we expect to know its position with an accuracy of $5 \mathrm{~m} \AA(1 \sigma)$ corresponding to typically $75 \mathrm{~km} \mathrm{~s}^{-1}$, at $20 \AA$.

Further improvements can be made when lines are observed in both first and second order. The angle of incidence on the grating for the particular observation can be independently solved by forcing the corresponding first and second order lines to have the same observed wavelength.

The pointing accuracy of the XMM-Newton telescope is 1.5 arcsec, which corresponds to about $3 \mathrm{~mA}$ in the dispersion coordinate. In addition, the emission lines of most coronal sources, due to the violent processes in the coronae and/or the existence of companion stars have velocity uncertainties of $40-50 \mathrm{~km} \mathrm{~s}^{-1}$ or about $3 \mathrm{~mA}$. The accuracy of our current wavelength calibration is very close to this theoretical limit of about $4.5 \mathrm{~mA}$.

\section{Effective area}

\subsection{Effective area model and ground calibrations}

The effective area is a combination of the mirror effective area, the grating efficiency and scattering and the detector quantum efficiency together with any data selection applied. In Fig. 33 the results of the end-to-end tests at the Panter facility are displayed including the expected effective area. The data are corrected for the finite source distance. 


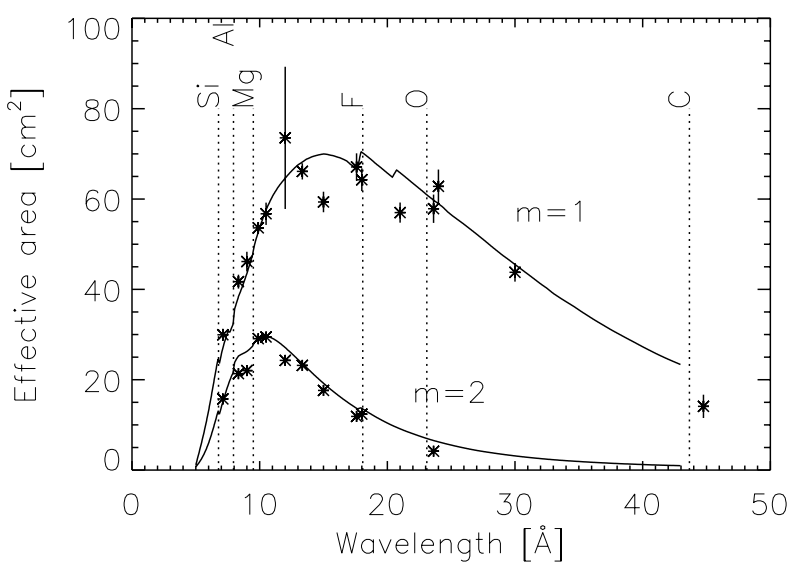

Fig. 33. Effective area verified preflight by end-to-end measurements at the Panter facility (den Herder et al. 2001). This can be compared with the measured in flight effective area as verified on celestial sources (Fig. 34). The model is indicated by the solid line. Sharp features are caused by either atomic absorption edges or differences between different CCD Al layers. The broken lines indicate the relevant atomic edges. The asterisk symbols show the measured data points.

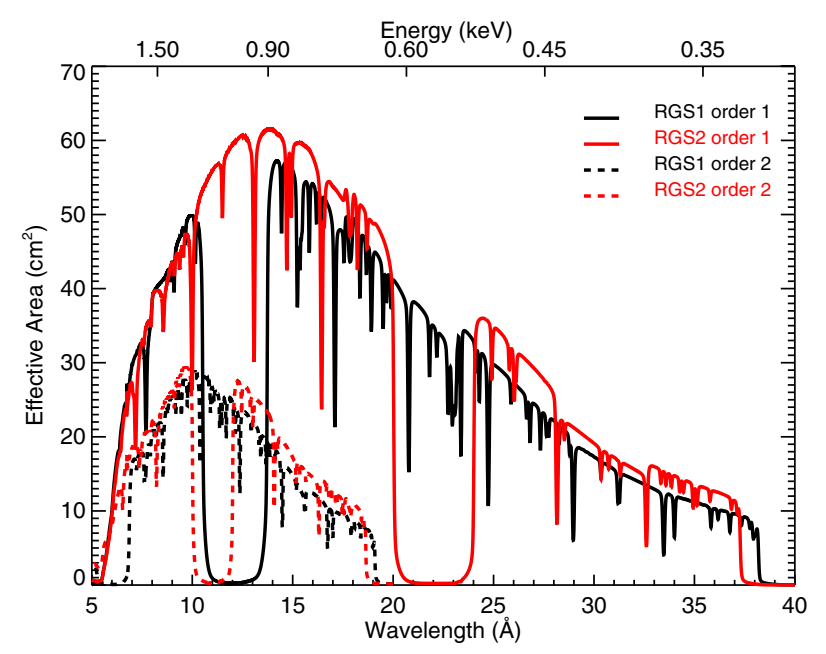

Fig. 34. Effective area for RGS1 (black) and RGS2 (red) (two orders) as calculated by the SAS, based on in flight calibrations on celestial sources. Apart of the two failing chains (CCD4 in RGS2 and CCD7 in RGS1) the CCD boundaries can be easily identified. In addition the effect of hot pixels and columns can be seen.

These results indicate a $10 \%$ accurate calibration of the effective area over the 10-25 $\AA$ range. At shorter wavelengths there is a steep gradient in the grating efficiency, resulting in a significantly larger uncertainty of the effective area. At the longest wavelength the CCD QE is less accurately known, up to $40 \%$ (see Fig. 9). The longest wavelength point in Fig. 33 is well beyond the maximum wavelength of $38 \AA$ for the in flight configuration of the RGS instruments.

\subsection{In orbit scaling}

Considerable effort was invested to improve the effective area in orbit by observing continuum sources with good statistics. A total of 1 Ms observations of Mrk 421, PKS 2155-304 and other selected sources (e.g. Vela pulsar, Sco X-1 off axis) are used. The resulting area result is shown in Fig. 34. All modifications of the area model are needed due to either uncertainty in the model response or items not fully included in the calibrations.
An unexpected vignetting of the beam was deduced by comparison of the RGS1 and RGS2 response. After launch it turned out that the effective area for RGS1 was less than that for RGS2 and that it scaled with the angle $\beta$ of the dispersed rays $\left(A_{\mathrm{eff}}(\mathrm{RGS} 1) / A_{\mathrm{eff}}(\mathrm{RGS} 2)=1\right.$ for $\beta=0.036$ and 0.8 for $\beta=0.072)$. This dependency is consistent with a partial blocking of the through beam between the mirrors and the RFC and is explained by a $180^{\circ}$ rotated baffle of the grating assembly around the optical axis. The purpose of this baffle is to isolate the RGA thermally from its environment. The exit opening is asymmetric with respect to the mirror optical axis to allow an unblocked view to the RGS camera. Although this should have been checked during integration, from a manufacturing point of view rotation over $180^{\circ}$ around the optical axis cannot be excluded. The RGS1 effective area is corrected for this using a linear function of $\beta$.

The $Q E$ of CCD2 in RGS2 was modified based on continuum spectra of Mrk 421 and PKS 2155-304. In the initial data an unexpected jump in the spectrum was observed for CCD2 in RGS2. The observed loss of QE can be explained and is modeled with an additional $40 \mathrm{~nm}$ layer of $\mathrm{SiO}_{2}$ on the CCD. This layer does match the observed absorption in both first and second orders. Why such an extra layer of $\mathrm{SiO}_{2}$ should exist on this CCD however, is not clear, and there may be another (unknown) cause for the observed loss in QE.

Systematic flux variations (up to $10 \%$ of the flux) on scales of a few Ångstroms, as function of wavelength were observed for sources which are expected to show smooth, power law spectra (e.g. PKS 2155-304, Mrk 421). These deviations from the expected power laws are attributed to unaccounted variations of the effective area. To correct these flux variations, we took high statistics observations of Mrk 421 in orbits 84, 259, 546, 720 and 1084 and fitted absorbed power law spectra to the observations in the interval from 10 to $25 \AA$. For each spectrum different power law indices and normalizations were allowed, but only one $\mathrm{N}_{\mathrm{H}}$ column density for the interstellar absorption. Thus the source was allowed to vary between observations, but the interstellar absorption remained constant. The same set of parameters was fitted to both RGS1 and RGS2 spectra. It is assumed that in the interval between 10 and $25 \AA$ the effective area of the RGSs is known best, and that in this RGS wavelength interval Mrk 421 shows a smooth power law spectrum.

Next the fitted power law and absorption model parameters were frozen and 12th order Chebychev polynomials (which could follow the few $\AA$ scale variations) were fitted over the full 5 to $38 \AA$ interval to the fit residuals for each RGS separately, but identical for all observations. These Chebychev polynomials are now used as corrections on our effective area and are included in the SAS calibration files.

The second order effective area is normalized using the first order spectra, because the main emphasis of the ground calibrations has been the first order only. The effect of this correction, which is similar for RGS1 and RGS2, is typically of the order of $20 \%$ except at very short wavelengths (see Fig. 35) and is well within the uncertainties of our theoretical model.

\subsection{Instrumental absorption edges}

Oxygen edge: the instrument turned out to have a significantly larger instrumental oxygen edge in orbit than anticipated. Some of this is due to oxygen contamination of the optics (see Fig. 24 for example), but a major contribution is on the detector itself. Following launch the detector has always been cooled: before the detector was opened its temperature was $-50^{\circ} \mathrm{C}$ while 


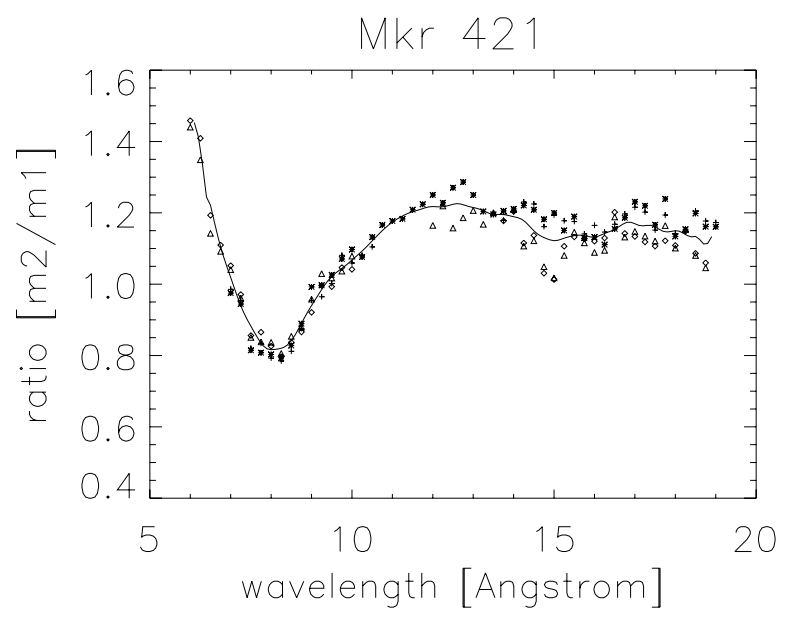

Fig. 35. Correction factor for the $m=-2$ spectra relative to the $m=-1$ spectra (identical for RGS1 and RGS2).

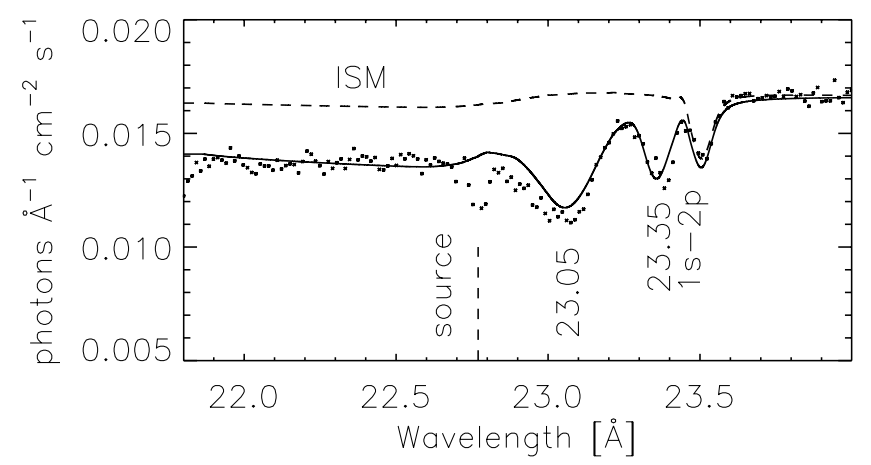

Fig. 36. Combined spectrum around the O-edge for Mrk 421 and PKS 2155-304 with the expected absorption from the ISM alone (dashed line) and in combination with the instrumental component (solid). The feature at $22.7 \AA$ is not visible in other sources and is therefore of astrophysical origin.

following the opening of the detector the temperature dropped to $-80^{\circ} \mathrm{C}$ and subsequently around orbits 532-537 it was cooled to $-110^{\circ} \mathrm{C}$. In practice this means that any contaminating water ice, present on the detector body during launch, would have remained on the detector and has never evaporated.

In orbit the oxygen contamination has been determined using relatively smooth continuum spectra. The oxygen absorption feature is illustrated in Fig. 36 where the spectrum for the combined data of two BL Lac objects with low extinction are shown (172 ks for Mrk 421 and $346 \mathrm{ks}$ for PKS 2155-304) as well as the expected interstellar medium (ISM) extinction. There is a clear mismatch. Comparing these data with sources with high extinction (Sco X-1) it is possible to deduce the instrumental response over the O-edge. The result is shown in Fig. 37. See de Vries et al. (2003) for details. In the observed data the sharp features in this figure will be convolved with the broader instrument response. Applying this instrumental correction to the low extinction data improves the description considerably (see Fig. 36). The feature at $22.77 \AA$ visible in this figure is not present in a number of other spectra (Sco X-1, 4U 0614+19) and is therefore assumed to have an astrophysical origin.

Fluor and magnesium edges: on the CCD detectors there is a $\mathrm{MgF}_{2}$ layer, which electrically isolates the optical Al filter from the $\mathrm{CCD}$. This layer will give rise to $\mathrm{Mg}$ and $\mathrm{F}$ absorption edges in the spectrum. By taking combinations of deep spectra of continuum power law sources (Mrk 421, PKS 2155-304)

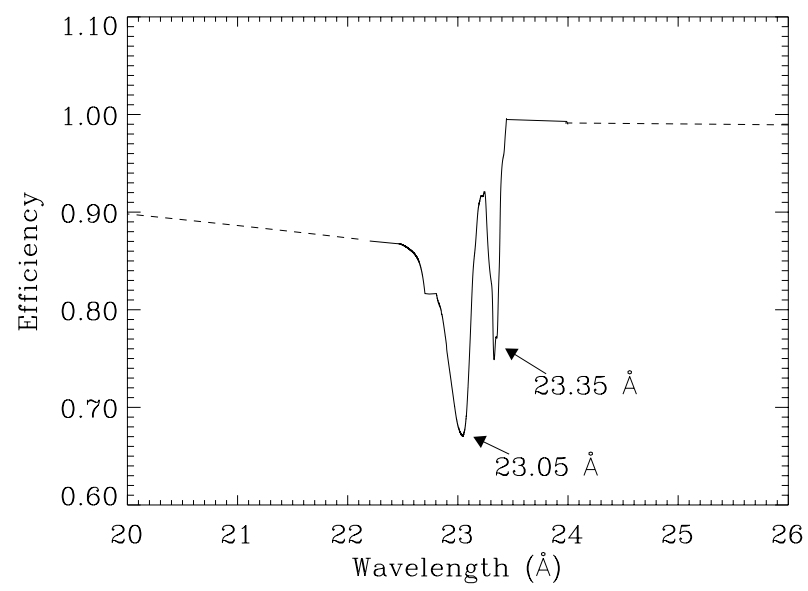

Fig. 37. Instrumental efficiency around the O-edge.

and assuming no astrophysical features were present around the $\mathrm{Mg}$ and $\mathrm{F}$ edges, the details of these edges were measured. The $\mathrm{Mg}$ edge follows with sufficient accuracy the edge predicted by Henke et al. (1982), but the F edge shows deviations. The depth of the edge agrees with the depth predicted by Henke and the known thickness of the $\mathrm{MgF}_{2}$ layer, but the shape of the edge is different. This fitted shape is included in the effective area calibrations used by the SAS.

\subsection{Hot pixels}

In hot pixels the charge sometimes tends to overflow along the column to neighboring pixels. When pixels are only occasionally hot during an observation, these pixels are not recognized as such, but the set of neighboring pixels in a frame will be recognized by the onboard processing, and discarded as being a "complicated" event or cosmic ray. Such discarded pixels are not individually flagged, but only the average decrease in effective area of the whole CCD is recorded. Hence holes, or "cool" pixels, appear in the processed spectra as narrow "absorption" features, due to the unrecognized loss of effective area for some columns. A typical "cool" column is shown in Fig. 38.

When searching for weak absorption features, it is important that these effects are taken into account. A list of pixels and columns is maintained in the calibration files (the "COOLPIX" ccf). In the ground processing software (SAS) there is an option to include or exclude these known pixels. For individual observations, redundancy of both RGS instruments may allows us to identify these columns in the individual instruments. In addition spectra of first and second order are compared to reject absorption features which occur at the same spot on the CCD, which is at half the wavelength in second order. Furthermore the multiple pointing mode (Sect. 11.2) helps to mitigate this problem.

\subsection{Contamination}

Prior to launch the camera was purged with clean $\mathrm{N}_{2}$ and its door was sealed. Between this sealing and the launch many months passed. In this period water vapor may have entered the cavity of the CCD detectors, especially during the time on the launch platform in the very warm and humid air at the tropical launch site of Kourou, French Guiana.

Following the launch the instrument was passively cooled to $-50{ }^{\circ} \mathrm{C}$ and stayed at this temperature until the opening of the detector door. Hence it is not surprising that a considerable oxygen edge is observed in the instrument response (see Sect. 9.3). 


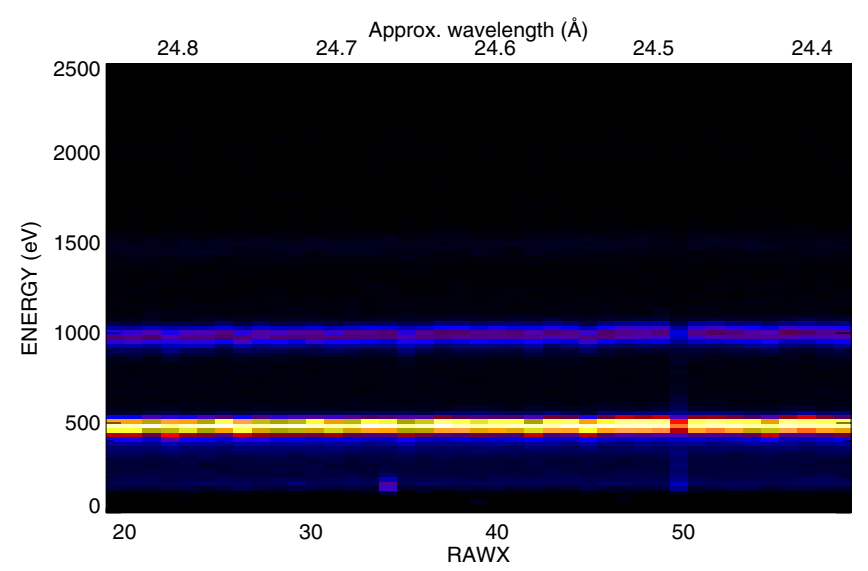

Fig. 38. Signature of a cool column on CCD4 of RGS1 (1 Ms Mrk 421 data summed in detector space in a plot of pulse-height (Energy) versus dispersion axis. Top curve is the second order of the dispersed spectrum, while bottom curve shows first order. A "cool" column can be recognized around RAWX $=50$, or about $24.5 \AA$ in first order. This column extends into second order at the same position, but at half the wavelength. Top scale is the approximate wavelength of the first order only.
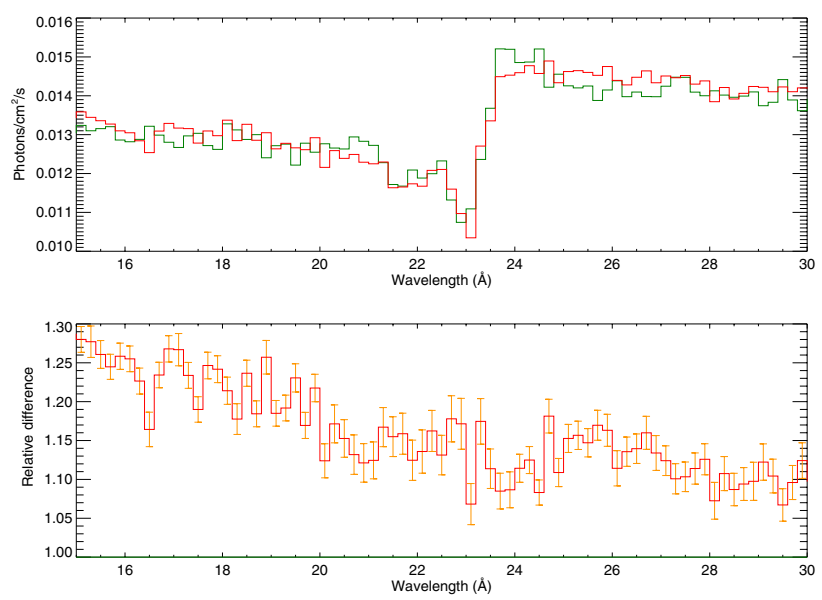

Fig. 39. Stability of the instrumental oxygen edge: comparison of spectra corrected for a change in slope and normalization (top) and ratio (bottom) of Mrk 421 data in orbit 84 (red) and orbit 2002 (green). The spectra show the uncorrected instrumental edge, which is normally corrected for during default data processing. The slight linear trend in the relative difference plot (bottom) is caused by small changes in the source power law spectrum over time.

Although the instrument has the capability to be heated up to around $45^{\circ} \mathrm{C}$ this has not been exercised as it is virtually impossible to predict accurately what happens to the instrument which has stayed cool for over 14 years. In addition any contaminants, when evaporated, will largely stay inside the telescope tube and hence will condense again at a later stage.

The depth of the oxygen edge is very stable over time (see Fig. 39). Apparently the contamination with water ice indeed was a single event, and a single calibration (Sect. 9.3) suffices to correct for this effect.

However, monitoring of two stable sources (RX J1865.53754 and the Vela pulsar) shows that the response of the instrument at long wavelengths suffers from loss of effective area, which increases over time. This is thought to be caused by a second contamination component: out-gassing of hydrocarbons by the carbon-fiber structures of the telescope tube, although great

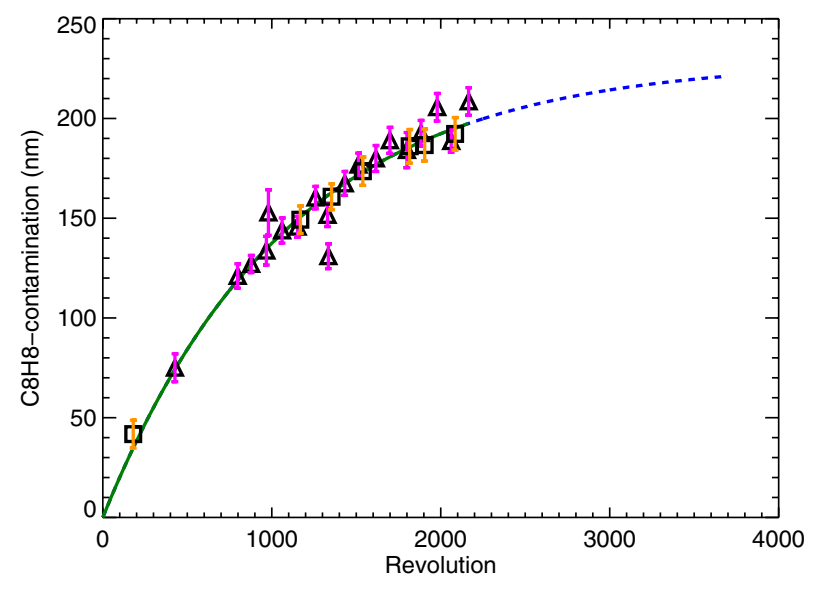

Fig. 40. Contamination history given in units of C8H8 thickness, $\rho=$ $1.11 \mathrm{~g} / \mathrm{cm}^{3}$. Triangle data points are the RX J1856.5-3754 data points, while the square points are the Vela pulsar data points. The broken line is the prediction of the contamination for the near future.

care has been taken with selection of the materials. A special cold section at $-80^{\circ} \mathrm{C}$ is present in the telescope tube to freeze contaminants, and an out-gassing vent port is present to release any remaining contaminants to open space. However contamination of the RGS CCD detectors cannot be prevented because the CCDs are the coldest structures $\left(-110^{\circ} \mathrm{C}\right)$ and completely open to the telescope tube. By contrast, the EPIC cameras usually have optical blocking filters in front of them, which are at higher temperatures, and protect their CCDs. In addition, contaminating layers may also form on the optics (mirror and gratings), which will have the same effect.

Assuming perfect stability of the calibration sources RX J1865.5-3754 and the Vela pulsar at long wavelengths, the thickness of the contaminating layer can be calibrated. Assuming a type of hydrocarbon as $\mathrm{C}_{8} \mathrm{H}_{8}$ to be representative for the contaminating compound(s), Fig. 40 shows its measured thickness with time. After an initial increase of the carbon layer on the detector, the increase now levels off as the mission progresses. For revolution 2200 a reduction of $47 \%$ of the detector response at $35 \AA$ is observed. At shorter wavelengths, this reduction is smaller e.g. $35 \%$ at $30 \AA$, and $24 \%$ at $25 \AA$. This reduction of effective area is taken into account during standard processing.

Using the onboard calibration sources in principle the contamination of the CCDs can be verified independently. This is however complicated because the calibration source, a $\mathrm{Cm}^{244} \alpha$ emitting source, is also cool and contaminated. Hence contamination of the source or of the target will effectively lower this count rate more than is expected based on the radioactive decay time of the source itself. Especially the alpha particles of the radio-active source, which are generating the X-ray fluorescent photons on the F and Al targets, are very susceptible to small amounts of contamination. This is illustrated in Figs. 41 and 42. In addition, the hydrocarbon contamination will mainly affect the longest wavelengths but the Fluor-K line and the Al-K line are at shorter wavelength (18.30 and $8.33 \AA$, respectively) and hence far less sensitive to contamination than the astrophysical calibration sources at 32 to $37 \AA$.

\subsection{Pile-up effects}

Pile-up, which is the process of two X-ray events hitting the same or neighbouring pixel(s) within one readout frame, has the 


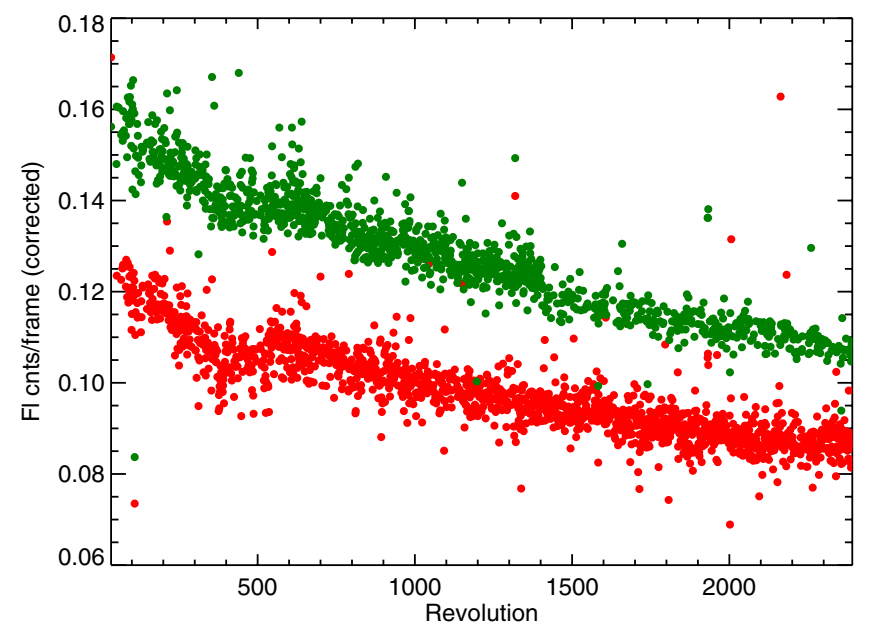

Fig. 41. Onboard calibration source count rate for the Fluor source after correction for source decay time and CCD hot pixels and columns. Red data points is RGS1, green data points is RGS2.

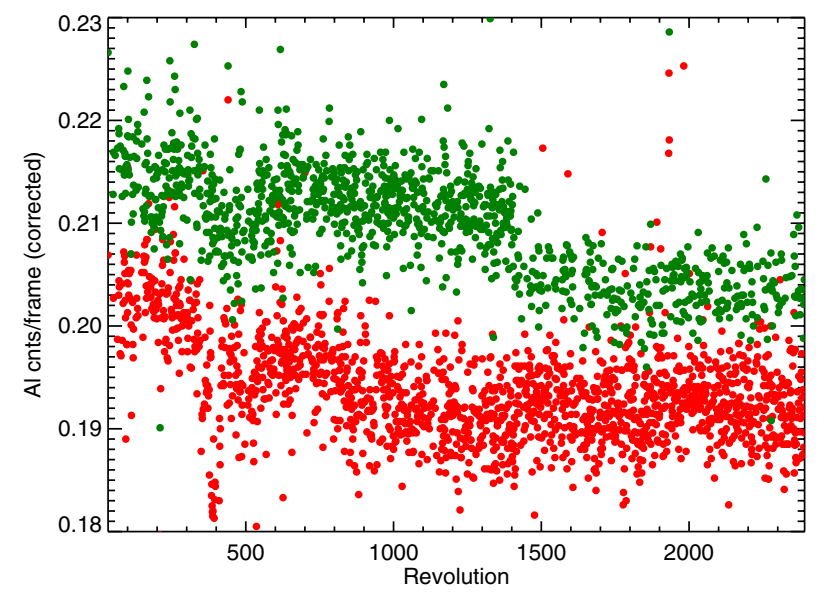

Fig. 42. Onboard calibration source count rate for the Al source after correction for source decay time and CCD hot pixels and columns. Red data points is RGS1, green data points is RGS2. The drop in RGS2 count rate around orbit 1405 is due to the switch to single node readout. A smaller drop at the same time is also observed for the fluor source (Fig. 41).

effect on the effective area of moving two events from e.g. first order into one event in second order. Consequently the effective area of the first order will decrease, while considerable background is added to second order. Fortunately pile-up is negligible for most sources.

Taking observations using different CCD integration times, by using different total number of CCDs in the readout chain, on the same constant source, pile-up can be measured. This way it is estimated that pile-up for the very bright source of the Crab is maximally $14 \%$ around $12 \AA$ wavelength (Kaastra et al. 2009), when using all CCDs in the default spectroscopy double node readout mode. However, Crab is an extended source. For the bright point source Mrk 421 in a flux state of 450 photons $\mathrm{m}^{-2} \mathrm{~s}^{-1} \AA^{-1}$ at $\lambda=25 \AA$, maximum pileup is estimated at about $1 \%$ for $\lambda=16-18 \AA$ for double node readout. For RGS2 in single node readout, pile-up is worse at about $2.4 \%$. Most sources are much weaker than Mrk 421, and hence pile-up can be ignored.

For very bright sources (e.g. Sco X-1, Cyg X-2) however pile-up can have dramatic effects. When there is considerable pile-up, neighbouring pixels can combine and form "complex" patterns, which will be discarded onboard. Hence, holes will appear in the spectrum. There is no way to correct for those. Best is to limit pile-up by using the RGS small window mode (see Sect. 11.2), and/or using only a limited number of CCDs in the readout chain.

\subsection{Effective area accuracy}

The absolute scale of the RGS effective area was calibrated on the well known source of the Crab pulsar and nebula. This calibration has been described in detail by Kaastra et al. (2009). It is estimated that the absolute effective area scale of the RGS is accurate to within the accuracy of the adopted continuum model for the Crab, which is about $10 \%$.

Contrary to most sources, the Crab is an extended source. This has been taken into account into the effective area calibrations, which means that the instrumental models for off axis effective area and cross dispersion scattering were incorporated in the effective area analysis. In addition custom selections had to be applied in the pulse-height versus dispersion coordinate space to prevent inclusion of too much CCD background at long wavelengths and low pulse-height values. Details of this analysis are given in Kaastra et al. (2009). For on-axis point sources these effects do not play a role. Crab is also a bright source, which means that pile-up has an effect. As mentioned before, this effect was taken into account.

The redundancy between RGS1 and RGS2 can be used to obtain some idea about small scale systematic uncertainties in the effective area, which define the sensitivity for detecting weak absorption structures. We start with the fluxed spectra $S_{\lambda}$, in which all known exposure, bad columns and pointing history effects have been taken into account in the used response. The $\lambda$ bins of the spectrum are sufficiently narrow that they are of the same order as the wavelength range of individual CCD pixels, such that uncalibrated individual pixel effects will show up the fluxed spectrum. We know that on larger scales $(>1 \AA)$ residual uncalibrated differences exist in effective area between corresponding bins of RGS1 and RGS2 and hence between the computed fluxes in the spectra. Small scale spectral structures will be retained in the difference $\Delta S_{\lambda}$ between the original spectrum $S_{\lambda}$ and the $1 \AA$ smoothed (convolved) spectrum $\mathrm{C}_{1 \AA}\left(\mathrm{S}_{\lambda}\right)$ :

$\Delta \mathrm{S}_{\lambda} \equiv \mathrm{S}_{\lambda}-\mathrm{C}_{1 \AA}\left(\mathrm{S}_{\lambda}\right)$.

In effect this is a high spatial frequency filtering of the spectrum, which keeps the narrow features, by subtracting the smoothed spectrum in which all features have been smoothed over a sufficiently $(1 \AA)$ wide area. For a featureless spectrum, the $\Delta S_{\lambda}$ should follow the noise statistics of the spectrum, such that

$\sigma^{2}=\frac{\sum\left(\Delta \mathrm{S}_{\lambda}-\overline{\Delta \mathrm{S}_{\lambda}}\right)^{2}}{N}$

where $\overline{\Delta \mathrm{S}_{\lambda}}$ is the average value for $\Delta \mathrm{S}_{\lambda}$, which should be around zero, $\sigma^{2}$ the variance of the distribution, and $N$ the number of spectral points. For the individual spectral points $S_{\lambda}$ the statistical error $\sigma_{\mathrm{s}}$ is known from the photon statistics of the observation. For a featureless spectrum the average error should be identical to the computed sigma: $\sigma=\overline{\sigma_{\mathrm{s}}}$, unless systematic effects play a role.

However, we do not know if the spectrum is really featureless. To get an idea of systematics we look at the difference between RGS1 and RGS2. The individual spectra should follow 
Table 11. Differences between RGS1 and RGS2 as function of signal to noise ratio per pixel.

\begin{tabular}{ccc}
\hline \hline$S / N$ & $\begin{array}{c}\text { Difference } \\
\text { width }\left(\sigma_{\mathrm{s}}\right)\end{array}$ & $\begin{array}{c}\text { Outliers }^{b} \\
(\%)\end{array}$ \\
\hline 6.7 & 1.01 & 0.2 \\
36 & 1.09 & 0.3 \\
69 & 1.41 & 3.7 \\
\hline
\end{tabular}

Notes. Differences outside normal Poisson statistics reflect the systematic uncertainties in the effective area accuracy. ${ }^{(a)}$ Width of distribution of differences expressed in statistical uncertainty $\sigma_{\mathrm{s}}$ of the spectrum. Offsets (constant difference) are ignored. ${ }^{(b)}$ Percentage of pixels outside of the $3 \sigma_{\mathrm{s}}$ statistical uncertainty.

the same true features, and hence these features are eliminated from the difference:

$\Delta \mathrm{S}_{\lambda_{12}} \equiv \Delta \mathrm{S}_{\lambda_{\mathrm{RGS} 1}}-\Delta \mathrm{S}_{\lambda_{\mathrm{RGS} 2}}$

The variance of this subtraction $\left(\sigma_{12}^{2}\right)$ is twice the variance of the individual $\Delta \mathrm{S}_{\lambda}$ :

$\sigma_{12}^{2}=\frac{\sum\left(\Delta \mathrm{S}_{\lambda_{12}}-\overline{\Delta \mathrm{S}_{\lambda_{12}}}\right)^{2}}{N}=2 \cdot \sigma^{2}$.

Table 11 shows $\sigma$ computed from the RGS1-RGS2 difference, expressed in units of error on the individual spectra points $\sigma_{\mathrm{s}}$ for the observed flux in Mrk 421, by combining different sets of observations to obtain different signal to noise ratios of the spectrum. It appears that, when signal to noise increases the $\sigma$ of the distribution becomes larger than the statistical error $\sigma_{\mathrm{s}}$ because systematic uncertainty effects, the pixel-to-pixel systematic errors, become more dominant. This means that a larger fraction of pixels will have values outside the statistical expected accuracy (the outliers). This effect should be taking into account when looking for weak line features close to the statistically expected threshold. For signal to noise ratios larger than 10 (100 counts per bin) these effects start to play a role. For signal to noise ratios of around 70, the statistical uncertainty is equal to the systematic uncertainty, translating into about a $1.5 \%$ uncertainty in short scale effective area variations.

\section{Instrumental background}

The instrumental background consists of several components, each with their own characteristics and temporal behavior. In the next subsections we will discuss the characteristics and behavior of these components, and the way background can be dealt with in the observational data.

\subsection{Soft proton background}

The major time dependent component of the instrumental background are the soft protons, trapped in the earth magnetic field and scattered through the XMM optical path. Apart from the potential of causing damage on the CCDs (see Sects. 6.4 and 5.2), soft protons mainly add a time variable background component on the detectors. Due to the scattering characteristics of the mirrors and gratings, soft protons will have the highest flux closest to the optical axis, on CCD9, decreasing towards CCD1. Selecting data cuts outside the parameter space of the normal X-ray dispersed spectrum on CCD9 provides a high quality monitor on the soft proton flux and the nature of the main instrumental background component. In orbit, the count rate on

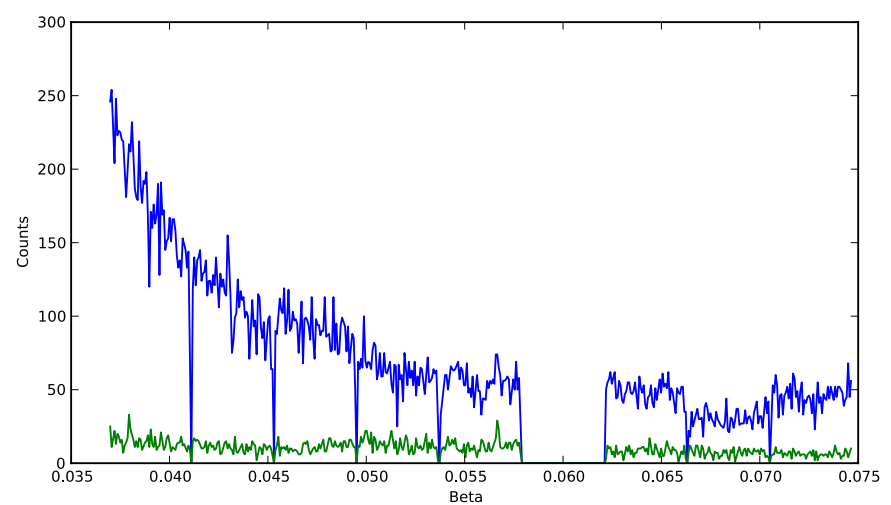

Fig. 43. Raw count rate distribution as function of CCD position along the dispersion direction for an off-axis region. The difference between a quiet period (green line) and a high background period (blue line) is in agreement with the expectations. The observations used are from AB Dor, RGS2, revolution 546 (high background) and revolution 668 (low background), with similar cross-dispersion and pulse-height selections for all CCDs.

RGS CCD9 is used as a radiation monitor to warn for high incident proton flux, and is used to activate the protection for the EPIC instruments. Figure 43 shows the nature of the soft proton flux as counts on the off-source positions, integrated over the full energy range as function of the dispersion axis, comparing a period of high soft proton activity with a period of low activity.

To improve the signal to noise ratio of the processed spectra, the count rate on CCD9 can be used to select periods of low soft proton background. For extended sources, count rates on CCD9 outside the normal spectra pulse height space are used as parameters to construct a background spectrum, based on spectra of empty fields with similar CCD9 count rate (soft proton) parameters. These sets of background spectra for various soft proton backgrounds are maintained as standard calibration files for the RGS processing software (SAS).

\subsection{High energy particle background}

Soft protons are low energy particles, scattered by the optical components. High energy particles, mainly from solar coronal mass ejection (CME) events, simply penetrate through all spacecraft structures, evenly illuminating the RGS CCD detectors. High flux events only occur occasionally and are usually predicted, in which case the instruments are switched off. Those events do however increase the amount of hot pixels and columns and CTI of the CCDs. At all times there is a low flux, high energy particle background. Many of these particles are detected and discarded as events well above the upper energy threshold due to their high energy contents, or are recorded on the CCDs as a track across many pixels, corresponding to the particle path across the CCD, and are discarded by the onboard processing as "complicated" events. The average effective area of the CCD is corrected for the pixels discarded in this way. It is thereby assumed that this background does illuminate the CCD evenly. The remainder of these events, which are not recognized as such add a low and flat background component.

\subsection{Detector background}

The CCD detectors produce low energy system noise and warm/hot pixels. These effects have been described before 
(Sects. 5.3 and 5.4). Applying the appropriate selections in data space and on pixel level, this background is minimized.

The calibration sources are a type of background introduced on purpose on the detector. This background is very constant and well known. The main part of this background is discarded by the appropriate selections in data space. The remaining part is perfectly modeled by the standard processing tools.

Another type of detector background is due to fluorescent lines generated by the high energy particles background in the detector housing. This background is variable. Fluorescent lines that can be recognized are the Al-K line because most of the material of the housing is aluminium and the gold-M line because there is a thin layer of gold deposited on the structures surrounding the CCDs. These lines can be recognized in CCD pulse height space only and are constant in dispersion and crossdispersion directions. Hence they do not show structure in the dispersed X-ray spectrum, but do add e.g. $0.02 \mathrm{cnts} / \mathrm{s}$ over the entire CCD detector for the gold-M line.

\subsection{X-ray background}

The sky itself provides a true X-ray background, consisting of numerous unresolved distant cosmic sources (see e.g. Gilli et al. 2007), and X-ray lines generated locally by e.g. the charge exchange mechanism in the earth vicinity in interplanetary space (see e.g. Cox 1998; Cravens 2000; Fujimoto et al. 2007). For the RGS the X-ray background can be treated as an extended source. X-ray lines from the background will be visible in the RGS spectra as very broad features which follow the effective area as function of dispersion offset. With standard background processing for point sources, which takes the background from off-axis positions in cross dispersion directions and applying the same selections in CCD pulse height space, the X-ray background is perfectly subtracted, since it is identical for source and off-source positions. When applying background models for extended source analysis, discrepancies between X-ray backgrounds from the sky positions from which the models are made with the observed sky position can keep some background signal in the spectrum. That signal however is usually very low.

\section{Instrument operations}

Instrument operations had a clear impact on the calibrations. Onboard hardware settings affect the efficiency with which the charge can be clocked through the CCD, the selected read-out speed affects the amount of pile-up, onboard event selection parameters influence the number of selected events to be transmitted to the ground and so on. In this section we present the operational aspects of the instrument that affected the calibrations. Table 12 lists the major changes in instrument operations. We first start with the operational conditions that affected the hardware configuration of the instrument.

\subsection{Instrument operational conditions}

Detector element failures: the read-out channels connected to two CCDs failed over the mission lifetime. The first failure (RGS2 CCD4) was traced back to a frequently used digital hardware switch (HS-202RH) whereas the second failure (RGS1 CCD7) could not be unambiguously traced back to a single hardware component and could also be due to failure in the CCD itself. Due to the complete redundancy between the two instruments, the full wavelength range is still covered. In particular around the $\mathrm{Ne} \mathrm{X} \operatorname{Ly} \alpha$ line and the $\mathrm{O}$ VII triplet this has significant impact.
Table 12. Major changes in RGS hardware operations and their scientific impact.

\begin{tabular}{|c|c|c|}
\hline Orbit & $\begin{array}{r}\text { Date } \\
\text { yy-mm-dd } \\
\end{array}$ & Event \\
\hline 24 & $00-01-25$ & first light RGS2 \\
\hline 26 & $00-01-30$ & $\begin{array}{l}\text { failure RGS2 CCD4 electronics } \\
19.9<\lambda<24.9 \AA\end{array}$ \\
\hline 30 & $00-02-06$ & first light RGS1 \\
\hline 45 & $00-03-08$ & $\begin{array}{l}\text { upload DPP code (19) for improved on- } \\
\text { board particle background data handling; } \\
\text { data }<\text { orbit } 45 \text { have wrong exposure }\end{array}$ \\
\hline 52 & $00-03-21$ & update IC code (S206): no impact \\
\hline 135 & 00-09-02 & $\begin{array}{l}\text { failure of RGS1 CCD7 } \\
(10.3<\lambda<13.6 \AA) \text {, RGS operations sus- } \\
\text { pended }\end{array}$ \\
\hline 147 & $00-09-26$ & operations resumed \\
\hline 168 & $00-11-07$ & $\begin{array}{l}\text { modification of bias settings of RGS to re- } \\
\text { duce part stress }\end{array}$ \\
\hline 171 & $00-11-13$ & RGS set to setup mode during perigee \\
\hline 192 & $00-12-25$ & RGS2:CCD2 bias back to before orbit 168 \\
\hline 361 & 01-11-29 & upload IC code (S207): no impact \\
\hline 363 & $01-12-01$ & upload IC code (S207): no impact \\
\hline 532 & $02-11-04$ & cooling RGS2 to $-115^{\circ} \mathrm{C}$ \\
\hline 537 & $02-11-13$ & cooling RGS1 and RGS2 to $-110^{\circ} \mathrm{C}$ \\
\hline 538 & $02-11-15$ & RGS2 CCD2: serial bias to $-2.7 \mathrm{~V}$ \\
\hline 584 & $03-02-15$ & CSG code (83): HTR $3 \times 72$ mode \\
\hline 765 & $04-02-13$ & 1st activation of current limiter RGS2 \\
\hline 786 & $04-03-24$ & 2 hot spots in RGS1:CCD1 \\
\hline 905 & 04-11-18 & RGS1 upload IC code (S208): no impact \\
\hline 907 & $04-11-22$ & RGS2 upload IC code (S208): no impact \\
\hline 1207 & $06-07-12$ & $\begin{array}{l}\text { lowering temperature electronics to limit } \\
\text { impact of current limiter }\end{array}$ \\
\hline 1389 & $07-07-11$ & RGS1 CCD2: serial bias to $-4.8 \mathrm{~V}$ \\
\hline 1408 & $07-08-16$ & single node read-out RGS2 \\
\hline 1671 & 09-01-24 & $\begin{array}{l}\text { upload CSG code (95): small window mode } \\
\text { became available to users after } 10-10-8\end{array}$ \\
\hline
\end{tabular}

Notes. At this moment, after 2009 no major changes have been necessary.

After the second failure it was decided to lower the clock voltages to reduce the load on various electrical components (orbit 168, see Table 12). This tuning resulted in a slightly modified instrument response which can be completely accounted for in the calibrations.

Perigee passage condition: during the initial phase the instrument was powered during the perigee passage. From orbit 171 this powering during perigee passage was stopped because there was concern about the de-rating of some of the electronic components related to the above mentioned failures. Because the instrument behaved stable over many years after this change, this concern was probably not justified.

Detector biasing: radiation damage at the CCD gate structure translates into a different biasing of the clocks used to transfer the charge through the CCD and thus results into a degradation of the charge transfer efficiency (CTE). By the capability of the instrument to set the clocking voltages in each CCD such degradation can be compensated. Apart from re-tuning to set proper clock voltages following the clock voltage reduction after the RGS1 CCD7 failure, changes in clock voltage settings have only been necessary for one CCD. The clock voltage of RGS2 CCD2 was modified in orbit 1389 (see Table 12).

Cooling: initially the detectors were operated at $-80^{\circ} \mathrm{C}$. This temperature was selected as a compromise between the charge transfer inefficiency (which gets better for temperatures as low 
as $-110^{\circ} \mathrm{C}$ or $-120^{\circ} \mathrm{C}$ ) and the potential contamination on the detector. Because there is no thermal filter between the detector surface and the XMM-Newton telescope tube, the structural element connecting the mirror platform and the detector platform, the camera will act as cryo pump for this volume. The relatively high operational temperature resulted in an increasing number of hot and flickering pixels during the first years in orbit. In orbit 532 and 537 the operating temperatures of the detectors were lowered to $-110{ }^{\circ} \mathrm{C}$ to reduce the fraction of hot pixels.

Current limiter RGS2: each CCD is read out simultaneously through two output amplifiers and only one CCD is read out at a time: while one CCD is read out, the others accumulate data. Upon completion of the read-out, the image section of the next CCD is transferred to the storage section and this next CCD is ready to be read out.

To handle the data streams each camera has two complete read-out chains, which control the clocking of the charge through the CCD, provide the analog to digital conversion and perform data selections to keep the telemetry within reasonable limits. Each read-out chain includes circuits to protect the rest of the system for potential failures. The dimensioning of one of the current limiters in one of the read-out chains (RGS2, node D) was too restrictive to cope with the de-rating of its electrical components beyond the planned lifetime. This happened after about 4 years. Initially the camera continued to operate properly as the current limiter was reset by the "power-on" of the CCDs following tripping of the current limiter. Due to the continued degradation of the components, the occurrence of this problem increased and started to affect the measurements; there was a need for more frequent resets. By lowering the temperature of the electronic components $\left(<4{ }^{\circ} \mathrm{C}\right)$ the current limiter was pushed back into its operating range. Further degradation of the components required an even lower temperature of the electronics after orbit 1408 (August 27, 2007). This could only be achieved by lowering the temperature of the full focal plane and this would affect the calibrations of the other instruments on XMM-Newton too much. Therefore it was agreed to disable one of the two read-out chains of RGS2 and read its charge through a single output node by changing the clock patterns. The only disadvantage is that this increases the read-out time of all CCDs by approximately a factor 2 resulting in a slightly larger dark current and a larger fraction of piled-up events. For most of the observations, however, the pile-up fraction is very small and therefore the impact is marginal. An unexpected result of this change in read-out was that the fixed pattern noise (see Sect. 5.2) disappeared.

\subsection{Operational settings}

In addition to these changes in the hardware configuration, there is a large set of onboard parameters and operating modes which may effect calibrations and/or data quality:

Lower level threshold: the unprocessed data stream from the CCDs is too large to be handled by the onboard electronics. Therefore we reject all bins with a contents consistent with the CCD system level plus dark current and (read-out) noise. This is to so-called rejection threshold.

Hot pixel and column rejection: in addition the pixel coordinates are compared to an uploaded hot pixel/hot column table and are rejected if they are listed in this table.

Event recognition: the remaining data are searched for valid $\mathrm{X}$-ray patterns. Events which spread their charge over an area which extends beyond a $2 \times 2$ pixel square are rejected (charged

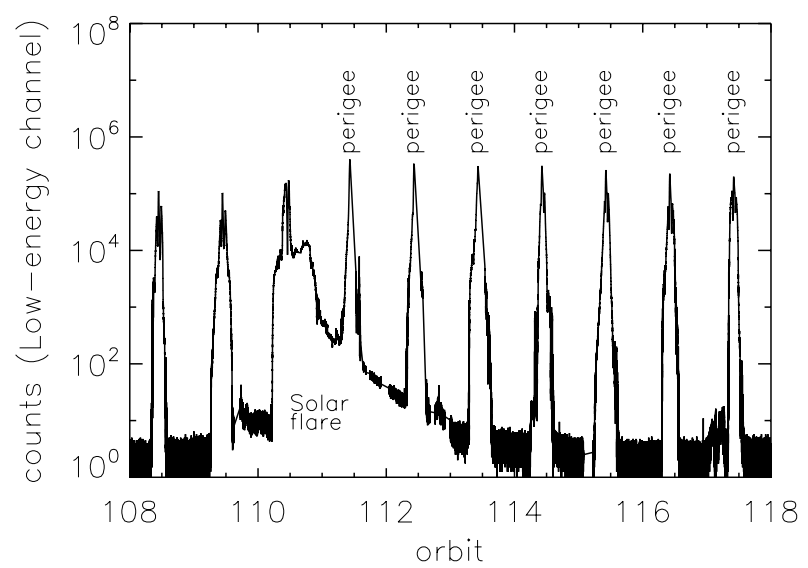

Fig. 44. Radiation environment for XMM-Newton, as observed by the radiation monitor in orbits $105-120$, including 10 perigee passages (indicated) and one of the strongest CME observed.

particles or piled-up events). After adding the charge for the remaining valid patterns, only reconstructed events are transmitted to the ground if their charge is above acceptance threshold and below upper threshold. The acceptance thresholds are set such that they approximately follow the lower boundary of the pulseheight distributions of first order dispersed spectral events. These thresholds are set per half of a CCD (CCD-node), and explain the increase of dark areas towards low $\beta$ angle in the bottom panel of Fig. 2.

Instrument modes: the read-out of each $\mathrm{CCD}$ can be controlled in great detail. For the RGS operations it turned out that $3 \times 3$ onboard binning of the pixels was a good compromise between the read-out speed, the number of hot columns, and the LSF. For high count rates one can reduce the effect of pile-up, meaning the effect of more than one photon in a bin during the data accumulation, by either reducing the number of CCDs to be read out, or by selecting a smaller region in the cross dispersion direction. This later option, the so-called "small window mode", is available from orbit 1671 onward.

Multiple pointing: despite all calibration efforts it cannot be excluded that there are small temporal unaccounted for CCD pixel to pixel variations in e.g. electric offset and/or charge loss due to evolving CCD damage. This varying CCD response effects the effective area by means of the event selections, and can affect the interpretation and identification of weak emission or absorption lines. By splitting long observations into observations with shorter observation time and using different pointing offsets in steps of $35 \mathrm{~m} \AA$ corresponding to steps of 15 arcsec, the detector response can be separated from real features in the observed source. Although this was feasible from the launch onward, from "call for proposals" AO08, orbit 1693, the so-called multi-pointing mode has been made available to general users. There is no overhead during re-pointing of the telescope, since all data, including the data obtained during the small slews of the telescope, can be used. Locations of X-ray events on the detector are converted into wavelength space, based on telescope pointing reconstruction at the moment of X-ray detection.

\subsection{Radiation environment}

The radiation environment is characterized by three distinct components: the orbital passage through the radiation belts, CMEs due to solar activity and regions in the orbit with trapped soft protons. In Fig. 44 two components are shown: the regular 
Table 13. Major solar activity, which affected the observations and/or operations.

\begin{tabular}{|c|c|c|c|c|c|}
\hline Orbit & $\begin{array}{r}\text { Date } \\
\text { yy-mm-dd }\end{array}$ & $\begin{array}{c}\mathrm{XMM} \\
\log [\mathrm{cnts} / \mathrm{s}]\end{array}$ & $\begin{array}{l}\text { Fluence }^{a} \\
\left(\times 10^{9}\right)\end{array}$ & $\begin{array}{l}\text { Hardness } \\
\text { ratio }^{b}\end{array}$ & Comment/duration \\
\hline 110 & $00-07-15$ & 4 & 1.2 & 0.48 & 3 revolutions \\
\hline $\begin{array}{l}169 \\
176\end{array}$ & $\begin{array}{l}00-11-10 \\
00-11-25 \\
\end{array}$ & 4 & 1.6 & 0.38 & $\begin{array}{l}\text { 1st burst, } 13 \text { revolutions } \\
2 \text { nd burst }\end{array}$ \\
\hline 239 & 01-03-30 & 3 & & & 9 revolutions \\
\hline $\begin{array}{l}375 \\
383\end{array}$ & $\begin{array}{l}01-12-27 \\
02-01-11\end{array}$ & 3 & & & $\begin{array}{l}\text { 1st burst, } 12 \text { revolutions } \\
2 \text { nd burst }\end{array}$ \\
\hline 433 & $02-04-21$ & 3 & & & 5 revolutions \\
\hline $\begin{array}{l}711 \\
723\end{array}$ & $\begin{array}{l}03-10-28 \\
03-11-20\end{array}$ & 4 & 1.0 & 0.28 & $\begin{array}{l}\text { 1st burst, } 15 \text { revolutions } \\
2 \text { nd burst }\end{array}$ \\
\hline 934 & $05-01-15$ & 4 & 0.8 & 0.25 & 5 revolutions \\
\hline 1052 & 05-09-07 & 3 & & & 5 revolutions \\
\hline 1282 & 06-12-09 & 3 & & & 6 revolutions \\
\hline 2106 & $11-06-09$ & 3 & & & 2 revolutions \\
\hline 2220 & $12-01-22$ & 3 & & & 4 revolutions \\
\hline 2242 & $12-03-06$ & 4 & & & 2 revolutions \\
\hline
\end{tabular}

Notes. ${ }^{(a)}$ Protons above $2 \mathrm{MeV}$ (lower limit). ${ }^{(b)} p(\geq 10 \mathrm{MeV}) / p(\geq 2 \mathrm{MeV})$, with $p$ number of protons.

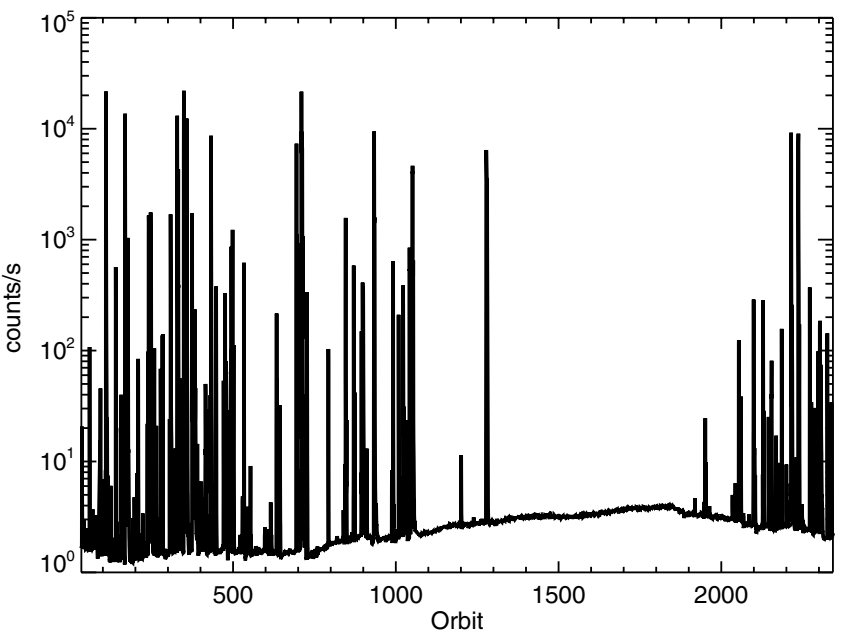

Fig. 45. Count rate outside the radiation belts registered by the $X M M$-Newton radiation monitor in the high energy "nhe2" channel (electrons: $1.3-1.75 \mathrm{MeV}$, protons: 9-37 MeV). This displays the solar activity (spiky structures due to solar flares) throughout the mission, showing the active sun at the start and after 10 years of mission and the solar minimum in between.

transfer through the radiation belts and the presence of one of the major CMEs during the mission lifetime.

Perigee passage: up to orbit 171 the instrument was kept switched on during the perigee passage and indeed the CCDs were completely flooded by charged particles (as could be deduced from the full frame images). After this orbit the instrument was switched off (with the exception of the digital electronics) to minimize possible damage by energetic charged particles of the different electronic components.

Solar activity: Fig. 45 shows all solar activity throughout the mission as recorded by the XMM-Newton radiation monitor. In Table 13 we list the major flares. We provide the combined maximum count rate for electrons between $130 \mathrm{keV}$ and $1.5 \mathrm{MeV}$ and protons between 1 and $4.5 \mathrm{MeV}$ during the increased solar activity. Clearly the strong flares last for several up to 15 revolutions. As is indicated in this table, XMM-Newton has experienced 5 major solar flares with $\log (\mathrm{cnts} / \mathrm{s}) \geq 4$. With use of the
OMNI data ${ }^{2}$ we have calculated the fluence and hardness ratios of these flares. Due to the incompleteness of the data (telemetry) we can only provide lower limits for the fluences but these are reasonably representative as the number of missing data points is limited. For the first 4 major flares the fluences and hardness ratios are given in the table. All other flares are significantly weaker. In Sect. 5.2 we described the effect of these flares on the detector response.

Soft protons: in addition to these expected radiation components "clouds" of soft protons are present in the environment. Typically their occurrence increases closer to perigee but they can occur in short bursts all over the orbit. In Fig. 46 we show the typical count rates during a random set of orbits. The count rates shown are data collected in the RGS instrument with pulse heights and positions on the detector where no X-rays dispersed from the gratings are collected (regions outside the source position, on CCD 9). Soft protons from outside will be absorbed by the satellite and detector housing but protons entering the telescope mirrors are scattered by the optics and subsequently recorded by the detector (see Sect. 6.4). Therefore this count rate is a reasonable accurate estimate of this background component.

Seasonal variations: the background rates during passage through the perigee belts shows a clear dependency on the season. During the course of the seasons the relative position of the orbit with respect to the Earth magnetosphere changes. This is illustrated in Fig. 47 where the count rate in RGS for $\lambda<7.7 \AA$ and a selection in the cross-dispersion direction $>1$ arcmin away from the source direction is shown for different phases of the orbit. During the first and last part of the orbit the instruments are switched off since during the perigee passing no science data can be recorded. In addition to reduced background closer to apogee we see also the seasonal effect of the impact of the Earth magnetosphere.

\section{Conclusions}

Continuous monitoring and special purpose calibration observations of the RGS instruments over the years, since launch, have yielded an accurate calibration and understanding of the

2 See http://omniweb.gsfc.nasa.gov/ow.html 


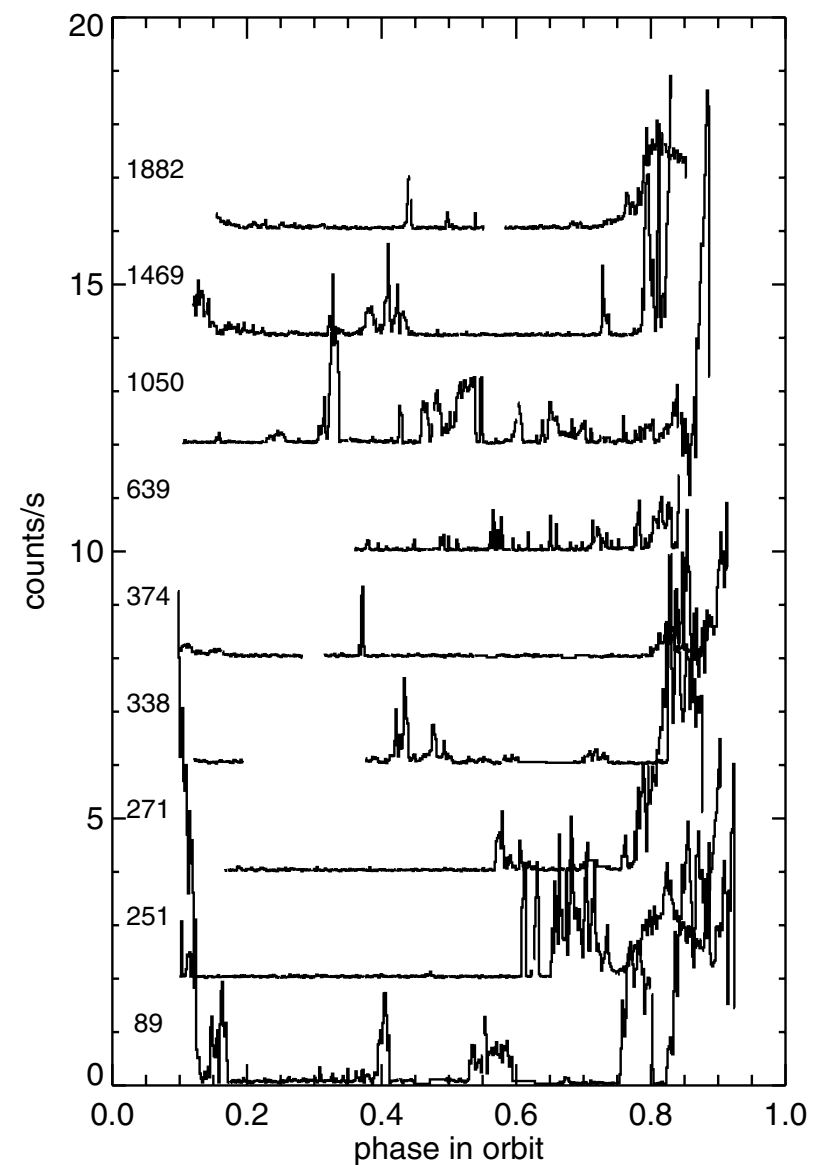

Fig. 46. Illustration of soft proton count rates per second in the RGS detector (CCD9 node C, RGS1) for 9 different orbits (displayed with an offset of 2 for clarity).

instruments. Although many instrument parameters were measured before launch, in flight measurements of celestial sources allowed a far more accurate assessment of these parameters. In addition the behavior of the instrument due to cosmic radiation, temperature and attitude variations, and aging, are now well understood.

The following major aspects of the RGS, described in this paper, can be summarized as follows.

- Detector performance: the CCD detectors are subject to damage by cosmic radiation and soft protons. The major effect is the appearance of hot pixels and columns. Cooling the detectors to $-110^{\circ} \mathrm{C}$ made most hot pixels disappear and has kept the amount of hot surface limited since then. Unfortunately two different CCDs were lost in the two instruments quite early in the mission, but overlap between the instruments still allows the full spectrum to be measured. Effects of increasing charge transfer inefficiency (CTI) are small $\left(<10 \times 10^{-5}\right)$ and appear not to play a role in the RGS.

- Wavelength scale: although the original calibration kept the wavelength scale accuracy within specification, improvements have been made by taking into account effects of the angle of solar illumination on the spacecraft and seasonal effects in pointing. The blind accuracy of the wavelength scale is now of order $6 \mathrm{~m} \AA$ in first order.

- Linespread function: the observed line shape accurately follows the model. The line center appears slightly narrower than originally predicted by the models. The response has been updated for that.

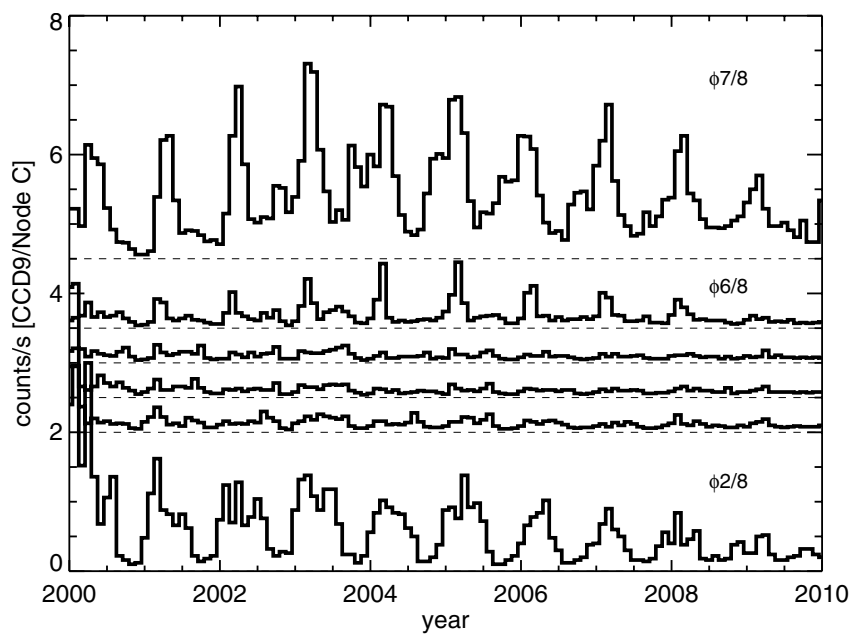

Fig. 47. Seasonal variations in the instrument background in counts per second on node C of CCD9, as function of orbit. The different phases of the orbit are shown from bottom to top with a different offset for clarity (indicated by the dashed lines). The phases for the perigee passage are omitted (1/8 and 8/8). The others are shown for increasing phase (bottom to top; for clarity the labels of phases $3 / 8$ to $5 / 8$ are left out). The large scale trend of increasingly smaller maxima in later years, shows the decreasing solar activity over the 11 year solar cycle.

- Effective area: the effective area is subject to bad pixels and columns and the two failed CCD chains (see Fig. 34). In addition, a constant absorbing layer of water and an increasing column of hydrocarbons decreases the effective area. At this moment (revolution 2500, almost 14 years in orbit), the effect of the hydrocarbons is a loss of $47 \%$ of effective area at $35 \AA$ and $24 \%$ at $25 \AA$. These effects are all taken into account in the standard calibration. The absolute accuracy of the effective area is estimated at $10 \%$.

- Sensitivity: deeper integrations in principle allow detections of weaker (absorption) lines, but do expose systematic instrumental structures, which may be confused with real astronomical features. Systematic structures can be identified by utilizing the intrinsic redundancies in the instrument (two RGSs, two orders). In addition, a multi pointing mode has been made available which is highly effective in decreasing the systematic errors and helps in identifying the remaining systematic structures. Ultimate sensitivity for weak absorption lines is reached when the statistical uncertainty matches the systematic uncertainty. This condition is met for absorption lines with depths of about $1.5 \%$ of the continuum.

The RGS instruments onboard XMM-Newton are in excellent shape, and have well exceeded their design life time (5 years). The actual performance has fulfilled the design goals. Detector (CCD) degradation due radiation and contamination has been limited and extrapolations show that detector performance will be quite adequate for at least another 20 years. Continuing calibrations and ongoing analysis of the instrumental behavior will keep this instrument in excellent shape in the years to come.

Acknowledgements. The authors like to thank the operations teams at ESOC and ESAC for their full support. In addition a number of colleagues have contributed significantly to the paper including in particular Ricardo Perez Martinez. We also like to thank Jacco Vink for his work on the effective area corrections, and Yan Grange for looking into extraction methods and background issues. Norbert Schartel gave some useful comments on a first version of the text. XMM-Newton is an ESA science mission with instruments and contributions directly funded by ESA Member States and the USA (NASA). SRON is supported financially by NWO, The Netherlands Organization for Scientific Research. 


\section{Appendix A: Calibration files}

Table A.1. Current calibration files.

\begin{tabular}{|c|c|c|c|c|c|}
\hline & Orbit & $\begin{array}{l}\text { Validation } \\
\text { start date } \\
\text { yy-mm-dd }\end{array}$ & $\begin{array}{r}\text { RGS } \\
1\end{array}$ & $\begin{array}{r}\mathrm{RGS} \\
2\end{array}$ & Comment \\
\hline \multirow[t]{17}{*}{ ADUConv } & 25 & $00-01-25$ & & 006 & values for launch \\
\hline & 30 & $00-02-06$ & 006 & 007 & values for launch \\
\hline & 105 & 00-07-09 & 007 & 008 & regular update \\
\hline & 114 & $00-07-23$ & 008 & 009 & regular update \\
\hline & 139 & 00-09-12 & 009 & 010 & regular update \\
\hline & 169 & $00-11-11$ & 010 & 011 & regular update \\
\hline & 191 & $00-12-25$ & & 012 & regular update \\
\hline & 259 & $01-05-10$ & 016 & 016 & regular update \\
\hline & 325 & 01-09-18 & 017 & 017 & regular update \\
\hline & 349 & 01-11-05 & 018 & 018 & regular update \\
\hline & 532 & $02-11-04$ & & 020 & RGS2 shortly at $-115^{\circ} \mathrm{C}$ \\
\hline & 536 & $02-11-13$ & 019 & 021 & update after cooling to $-110^{\circ} \mathrm{C}$ \\
\hline & 808 & 04-05-09 & 020 & 022 & regular update \\
\hline & 1400 & 07-08-01 & 022 & & regular update \\
\hline & 1408 & $07-08-17$ & & 025 & RGS2 single mode \\
\hline & 1839 & $10-01-01$ & 023 & 026 & regular update \\
\hline & 2021 & $11-01-01$ & 024 & 027 & regular update \\
\hline \multirow[t]{8}{*}{ CTI } & 1 & 00-01-01 & 002 & 002 & values for launch \\
\hline & 259 & $01-05-10$ & 003 & 003 & regular update \\
\hline & 532 & $02-11-04$ & & 004 & RGS2 shortly at $-115^{\circ} \mathrm{C}$ \\
\hline & 536 & $02-11-13$ & 005 & 006 & update after cooling to $-110^{\circ} \mathrm{C}$ \\
\hline & 807 & 04-05-09 & 009 & 009 & regular update \\
\hline & 1408 & 07-08-17 & & 011 & single mode read-out \\
\hline & 1839 & $10-01-01$ & 011 & 012 & regular update \\
\hline & 2021 & $11-01-01$ & 012 & 013 & regular update \\
\hline \multirow[t]{9}{*}{ BadPix } & 1 & $00-01-01$ & 012 & 012 & values for launch \\
\hline & 276 & $01-06-13$ & 013 & 013 & regular update \\
\hline & 308 & $01-08-14$ & 014 & 014 & regular update \\
\hline & 532 & $02-11-04$ & & 030 & after cooling to $-110^{\circ} \mathrm{C}$ \\
\hline & 536 & $02-11-13$ & 015 & & after cooling to $-115^{\circ} \mathrm{C}$ \\
\hline & 543 & $02-11-27$ & 030 & & after cooling to $-110^{\circ} \mathrm{C}$ \\
\hline & 1110 & 06-01-01 & 031 & 031 & regular update including 2 hot regions in RGS1:CCD1 \\
\hline & 1294 & 07-01-01 & & 032 & regular update \\
\hline & 1417 & 07-04-09 & 032 & & 2 hot regions are uploaded for orbit $>1416$ \\
\hline CoolPix & 1 & $00-01-01$ & 001 & 001 & values from launch \\
\hline QuantumEff & 1 & 00-01-01 & 015 & 016 & values from launch \\
\hline EXAFS & 1 & 00-01-01 & 005 & 005 & values from launch \\
\hline EffAreaCorr & 1 & 00-01-01 & 008 & 008 & values from launch \\
\hline ReDist & 1 & 00-01-01 & 004 & 004 & values from launch \\
\hline LineSpreadFunc & 1 & $00-01-01$ & 005 & 005 & values from launch \\
\hline CrossPSF & 1 & 00-01-01 & 004 & 004 & values from launch \\
\hline LinCoord & 1 & 00-01-01 & 008 & 008 & support science simulator \\
\hline \multirow[t]{2}{*}{ TemplateBckGnd } & 1 & $00-01-01$ & 006 & 007 & values at launch \\
\hline & 1408 & 07-08-17 & & 008 & RGS2 single mode \\
\hline SAACorr & 1 & $00-01-01$ & 001 & 001 & values from launch \\
\hline \multirow[t]{7}{*}{ HKParmint } & 1 & $00-01-01$ & 009 & 008 & values from launch \\
\hline & & 00-09-04 & 010 & & \\
\hline & & $00-11-07$ & 014 & 013 & \\
\hline & & 00-11-19 & 011 & 009 & \\
\hline & & $00-11-04$ & & 011 & \\
\hline & & $02-11-13$ & 012 & & \\
\hline & & $02-11-14$ & 013 & 012 & \\
\hline XRT AreaEff & 17 & $00-01-13$ & 008 & 009 & initial effective area verified in orbit \\
\hline XRT XPSF & 17 & $00-01-13$ & 014 & 014 & values from launch \\
\hline XMM BoreSight & 1 & 00-01-01 & 023 & 023 & values from launch \\
\hline XMM MiscData & 1 & 00-01-01 & 022 & 022 & values from launch \\
\hline
\end{tabular}

Notes. Details on CCF release are available on http://xmm2 .esac.esa.int/external/xmm_sw_cal/calib/rel_notes/index.shtml 


\section{References}

Aschenbach, B. 2007, in SPIE Conf. Ser., 6688, 66880I

Bopp, B. W., \& Fekel, Jr., F. 1976, AJ, 81, 771

Bräuninger, H., Burkert, W., Hartner, G. D., et al. 2004, in Optics for EUV,

X-Ray, and Gamma-Ray Astronomy, eds. O. Citterio, \& S. L. O’Dell, SPIE

Conf. Ser., 5168, 283

Cottam, J., Kahn, S., Rasmussen, A., Spodek, J., \& Paerels, F. 1998,

in Science with XMM, http://xmm.esac.esa.int/external/xmm_ science/workshop/1st_workshop/index.shtml

Cox, D. P. 1998, in IAU Colloq. 166: The Local Bubble and Beyond, eds. D. Breitschwerdt, M. J. Freyberg, \& J. Truemper (Berlin: Springer Verlag), Lect. Not. Phys., 506, 121

Cravens, T. E. 2000, ApJ, 532, L153

de Vries, C. P., den Herder, J. W., Kaastra, J. S., et al. 2003, A\&A, 404, 959 de Vries, C. P., Vink, J., Méndez, M., \& Verbunt, F. 2004, A\&A, 415, L31 den Herder, J. W., Brinkman, A. C., Kahn, S. M., et al. 2001, A\&A, 365, L7 Fano, U. 1947, Phys. Rev., 72, 26

Freyberg, M. J., Bräuninger, H., Burkert, W., et al. 2005, Exp. Astron., 20, 405
Fujimoto, R., Mitsuda, K., Mccammon, D., et al. 2007, PASJ, 59, 133

Gilli, R., Comastri, A., \& Hasinger, G. 2007, A\&A, 463, 79

Haberl, F., Turolla, R., de Vries, C. P., et al. 2006, A\&A, 451, L17

Henke, B. L., Lee, P., Tanaka, T. J., Shimabukuro, R. L., \& Fujikawa, B. K. 1982, Atomic Data and Nuclear Data Tables, 27, 1

Jansen, F. A., Heppener, M., Aarts, H. J. M., \& de Korte, P. A. J. 1989, in SPIE Conf. Ser. 1159, eds. C. J. Hailey, \& O. H. W. Siegmund, 105

Kaastra, J. S., Werner, N., den Herder, J. W. A., et al. 2006, ApJ, 652, 189

Kaastra, J. S., de Vries, C. P., Costantini, E., \& den Herder, J. W. A. 2009, A\&A, 497, 291

Kahn, S. M., Cottam, J., Decker, T. A., et al. 1996, in SPIE Conf. Ser. 2808, eds. O. H. Siegmund, \& M. A. Gummin, 450

Nicastro, F., Mathur, S., Elvis, M., et al. 2005, ApJ, 629, 700

O'Dell, S. L., Bautz, M. W., Blackwell, W. C., et al. 2000, in SPIE Conf. Ser. 4140, eds. K. A. Flanagan, \& O. H. Siegmund, 99

Prigozhin, G. Y., Kissel, S. E., Bautz, M. W., et al. 2000, in SPIE Conf. Ser. 4140, eds. K. A. Flanagan, \& O. H. Siegmund, 123

Rasmussen, A., Chervinsky, J., \& Golovchenko, J. 1999, in Columbia Astrophysics Laboratory, Document RGS-COL-CAL-99009

Rasmussen, A. P., Kahn, S. M., Paerels, F., et al. 2007, ApJ, 656, 129 